\title{
Métricas Invariantes à Esquerda em Grupos de Lie
}

\author{
Eliza Ramos de Andrade
}

\author{
dissertação de mestrado \\ INSTITUTO DE MATEMÁTICA E ESTATÍSTICA DA \\ UNIVERSIDADE DE SÃO PAULO
}

\author{
Área de concentração: Geometria \\ Orientador: Prof. Dr. Claudio Gorodski
}
Durante o programa de mestrado, a autora obteve apoio financeiro da FAPESP


Aos meus pais,

Trajano e Fátima 


\section{Agradecimentos}

Ao professor Claudio Gorodski, ser humano e profissional exemplar que, num momento importante de minha vida, acreditou em meu trabalho e proporcionou condições para que esta pesquisa fosse realizada. A ele agradeço não somente a confiança depositada em mim, mas também o incentivo incansável e sincero.

A FAPESP pelo incentivo financeiro concedido durante todo este projeto de mestrado e também de iniciação científica.

Aos componentes desta baṇca julgadora, por aceitarem ler este trabalho e contribuir para sua melhora.

Aos membros da banca do exame de qualificação, pelas contribuições: professores Dr. Antonio Carlos Asperti e Dr. Marcos Alexandrino.

Aos professores responsáveis pela minha formação acadêmica, em especial aos professores Dra. Mary Lilian Lourenço, Dr. Flávio Ulhoa Coelho, Dr. Antônio Carlos Asperti e Dra. Roseli Fernandez.

À minha família que sempre incentivou todas as decisões e projetos da minha vida.

Ao meu companheiro Pedro, pelo carinho, amor, respeito, admiração e, principalmente, paciência.

Aos meus amigos das turmas desta caminhada acadêmica, pelos ensinamentos, divergências e experiências. Em especial agradeço à Débora.

Ao time do Copas, por todas as boas risadas compartilhadas durante este período. 


\title{
Resumo
}

Neste trabalho estudamos propriedades e exemplos de grupos de Lie munidos de uma métrica Riemanniana invariante à esquerda. Procuramos relacionar propriedades do grupo e da álgebra associada com propriedades geométricas, como a curvatura. Estudamos a curvatura seccional, de Ricci e escalar de um grupo de Lie equipado com uma métrica invariante à esquerda. Outro grande objetivo é a busca por exemplos e para isso estudamos um grupo de Lie de dimensão três unimodular. Finalizando, estudamos o caso de métrica bi-invariante e o caso especial de curvatura estritamente positiva. Os resultados principais deste trabalho foram apresentados no artigo Curvatures of Left Invariant Metrics on Lie Groups [17] de John Milnor. O caso especial de curvatura estritamente positiva que também apresentamos aqui foi demonstrado por Nolan Wallach em [22]. A teoria necessária para esse estudo envolve algumas noções de grupos de Lie e geometria Riemanniana. Há, portanto, no decorrer da exposição, uma introdução aos cơnceitos básicos necessários à nossa teoria. Ao leitor interessado em uma exposição menos introdutória a esses conceitos recomendamos [23] e [4].

\begin{abstract}
This work has the purpose of presenting a study on Lie groups equipped with a left invariant metric. In special we search for relations between topological and algebraic properties of the group and the associated algebra and geometrical properties, such as curvature. We study seccional, Ricci and scalar curvatures and search for examples, so we start with a three dimensional unimodular Lie group equipped with a left invariant metric. Finally we study the case of bi-invariant metric and the special case of strictly positive curvature. The main part of this work is based on the article Curvatures of Left Invariant Metrics on Lie Groups [17] by John Milnor. For the results about strictly positive curvature that were presented here we refer to Nolan Wallach [22]. Some theory on Lie groups and Riemannian manifolds is necessary. During the text there are some basic facts, but for the interested reader we recommend [23] and [4].
\end{abstract}




\section{Introdução}

Um grupo de Lie é um grupo topológico que possui estrutura de variedade diferenciável de maneira que as operações multiplicação e inversão são diferenciáveis. Entretanto, por trás de tal definição formal há décadas de desenvolvimento matemático envolvendo principalmente equações diferenciais e geometria. Por volta de 1870, através de Camille Jordan e principalmente de Felix Klein, Sophus Lie entrou em contato pela primeira vez com a importância da relação entre teoria de grupos e geometria.

De 1870 a 1873 Lie e Klein se aproximaram mais. Klein estudava propriedades geométricas de um tetraedro complexo e Lie considerou este problema de uma maneira totalmente original. Para desenvolver este problema geométrico, Lie essencialmente definiu o que seria um sistema fechado de transformações que comutam, o que mais tarde foi chamado de grupo de transformações. Em particular Lie estudou transformações que diferem muito pouco da identidade, as chamando de transformações infinitesimais e definiu os espaços infinitesimais. A parceria entre Klein e Lie durou de 1870 a 1873. Em 1870 eles publicaram em conjunto dois trabalhos [13], [14], relacionados com o problema do tetraedro complexo, onde estão as principais idéias de Lie sobre grupos de transformações. Um grupo de transformações do plano é um caso muito particular do que mais tarde definiu-se grupo de Lie e, além disso, no espaço vetorial das transformações infinitesimais Lie introduziu um produto que o transforma em uma álgebra, a mais tarde chamada álgebra de Lie. Mais precisamente, Lie considerou um grupo analítico local de transformações de uma vizinhança aberta da origem no espaço complexo $n$-dimensional $\mathbb{C}^{n}$ e o relacionou com o espaço infinitesimal. Lembramos que na época não existia uma definição formal de grupo, mesmo com a teoria de Galois desvendada por Ludwig Sylow.

Outro aspecto importante na teoria de Lie é a influência de Carl Gustav Jacobi. Também em 1870, enquanto se aproximava de Klein, Lie estudava a teoria geral de equações a derivadas parciais. Lie se interessava nas aplicações a esta teoria da teoria de grupos, ele procurava uma analogia entre os resultados obtidos com Klein sobre tetraedros complexos e a teoria de Galois que ressurgia na mesma época, envolvendo-se assim com a teoria de Jacobi em equações diferenciais parciais e sistemas dinâmicos. Através de tais relações Lie apresentou, num trabalho de três volumes publicados entre 1988 e 1893 com colaboração de F. Engel [15], importantes resultados. Em termos atuais, o primeiro resultado importante de Lie é o conhecido resultado que mostra que a aplicação exponencial é um difeomorfismo de uma vizinhança da identidade da álgebra numa vizinhança da identidade do grupo. O segundo que mencionamos aqui é a correspondência entre grupos e álgebras de Lie. Observamos aqui que esses e outros resultados exibidos por Lie em Theorie de Transformationsgruppen são locais. O tratamento global foi 
desenvolvido mais tarde, principalmente por Élie Cartan e Hermann Weyl.

O estudo dos espaços infinitesimais, ou álgebras de Lie, foi desenvolvido por outros matemáticos, principalmente Wilhelm Killing, Cartan e Weyl. Killing, em quatro artigos [12] publicados entre 1886 e 1890, tinha como objetivo principal classificar as álgebras de Lie simples. Mas Cartan aprofundou e completou os estudos de Killing, em 1894 [5]. Weyl, por volta de 1949, tornou-se particularmente interessado nestes estudos pela influência da teoria da relatividade. Durante este período Weyl entrou em contato com o trabalho de Cartan sobre representação de álgebras de Lie, em particular também se envolveu com os resultados de Issai Schur sobre representação do grupo ortogonal especial $S O(n)$ e invariantes em $S l(n, \mathbb{C})$. Em 1924 Weyl publicou dois trabalhos [24], [25] que estendem o que Schur fez para o grupo simplético, generalizando o que Cartan fez sobre representações irredutíveis de uma álgebra de Lie simples. Muito ainda pode-se dizer sobre o desenvolvimento desta teoria, recomendamos [8] ao leitor interessado.

Neste trabalho vamos considerar um grupo de Lie como uma variedade Riemanniana, isto é, uma variedade diferenciável equipada com uma métrica Riemanniana. Em particular estudamos aqui métricas invariantes à esquerda. Há muitas vantagens em estudar esse tipo de variedade Riemanniana que possui boas propriedades como a homogeneidade e completeza. Em especial o cálculo da curvatura torna-se mais simples, o que gera interessantes relações entre propriedades da álgebra, propriedades topológicas do grupo e propriedades de curvatura (seccional, de Ricci e escalar). As Seções 2, 3 e 4 deste trabalho estudam tais relações. Parte-se na Seção 5 à busca de exemplos, com objetivo de aplicar os resultados das seções anteriores. Consideramos assim um grupo de Lie de dimensão três unimodular e estudamos alguns exemplos como $S l(2, \mathbb{R})$, $S O(3), S U(2)$, o grupo de Lorentz, o grupo Euclidiano, grupo de Minkowski, grupo de Poincaré e grupo de Heisenberg. Na Seção 6 estudamos propriedades de curvatura de tais exemplos. Na Seção 7 consideramos o caso não-unimodular e também exibimos resultados sobre a curvatura. A Seção 8 dedica-se ao estudo do caso bi-invariante, isto é, uma métrica invariante à esquerda e à direita, onde o resultado principal é a caracterizar um grupo de Lie munido de uma métrica bi-invariante. Na Seção 9 caracterizamos os grupos de Lie com métrica invariante à esquerda flat, isto é, que possuem curvatura identicamente nula. Finalmente na Seção 10 estudamos dois teoremas de Nolan Wallach sobre curvatura estritamente positiva, em especial mostramos que $S U(2)$ é o único grupo de Lie simplesmente conexo munido de uma métrica invariante à esquerda de curvatura seccional estritamente positiva. 


\section{Curvatura Seccional}

Seja $G$ um grupo de Lie de dimensão n, e seja $\mathcal{G}$ a álgebra de Lie associada a $G$, formada pelos campos de vetores $C^{\infty}$ invariantes à esquerda, ou seja, tais que $\left(d L_{g}\right)_{h}(X(h))=X\left(L_{g}(h)\right)=$ $X(g h)$, para todo $g$ e $h$ pertencentes ao grupo $G$; ou equivalentemente $d L_{g} \circ X=X \circ L_{g}$. Consideremos agora a variedade Riemanniana $(G, \mathrm{~g})$ onde $\mathrm{g}(\mathrm{ou}\langle\cdot, \cdot\rangle$ ) é uma métrica Riemanniana em $G$ invariante à esquerda, ou seja, tal que as translações à esquerda são isometrias. Seja $\left\{e_{1}, \cdots, e_{n}\right\}$ uma base para $\mathcal{G}$. Observe que:

$$
<e_{i}, e_{j}>_{x}=<e_{i}(x), e_{j}(x)>=<\left(d L_{x}\right)_{1} e_{i}(1),\left(d L_{x}\right)_{1} e_{j}(1)>=<e_{i}, e_{j}>_{1}
$$

para todo $x \in \mathcal{G}$, onde 1 é a identidade de $G$. Portanto os coeficientes da métrica com relação a uma base de campos de vetores invariantes à esquerda são constantes iguais ao valor na identidade. Assim, uma métrica invariante à esquerda em $G$ restrita ao espaço tangente à identidade $T_{e} G \simeq \mathcal{G}$ define um produto interno em $\mathcal{G}$. Reciprocamente, dado um produto interno $\langle\cdot, \cdot\rangle_{e}$ em $\mathcal{G}$, tal estende-se naturalmente a uma métrica invariante à esquerda em $G$ definindo-se:

$$
<u, v>_{x}=<\left(d L_{x^{-1}}\right)_{x}(u),\left(d L_{x^{-1}}\right)_{x}(v)>_{e}
$$

para $x \in G, u, v \in T_{x} G$. Isso significa então que a restrição $\left.g \mapsto g\right|_{T_{1} G}$ é uma bijeção entre o conjunto das métricas invariantes à esquerda e produtos internos em $\mathcal{G}$, e segue que a família de métricas invariantes à esquerda distintas em $G$ possui dimensão $\frac{1}{2} n(n+1)$. Veremos neste trabalho que dependendo de métrica que é considerada, obtemos propriedades do grupo (como a curvatura) totalmente distintas.

Um grupo de Lie $G$ equipado com uma métrica invariante à esquerda é uma variedade homogênea e completa. De fato, dados $p, q$ pontos de $G$, a translação à esquerda definida por $L_{q p^{-1}}$ é uma isometria que leva $p$ a $q$. Lembremos que pelo teorema de Hopf-Rinow, para verificar que a variedade Riemanniana $G$ é completa, podemos mostrar que $G$ é completo como espaço métrico, isto é, toda sequência de Cauchy converge em $G$, com a distância Riemanniana. Como $G$ é localmente compacto, escolhemos $\epsilon$ pequeno de maneira que a bola fechada de raio $\epsilon$ e centro na origem seja compacta. Como a translação à esquerda $L_{g}$ é uma isometria, a bola fechada de raio $\epsilon$ e centro $g$ também é compacta. Seja $\left(x_{n}\right)_{n \in \mathbb{N}}$ uma sequência de Cauchy em $G$. Então dado $\delta>0$ qualquer, existe $n_{0}$ tal que para quaisquer $m, n \geq n_{0}, \mathbf{d}\left(x_{n}, x_{m}\right) \leq \delta$, onde d denota a distância Riemanniana em $G$. Tomemos $\delta=\epsilon$. Temos assim que toda sequência de Cauchy em $G$ está contida em algum compacto. Logo possui subsequência convergente e, portanto, converge. 
Lembremos alguns conceitos sobre variedades Riemannianas. Seja $(M, \mathbf{g})$ uma variedade Riemanniana. O teorema de Levi-Civita garante que existe uma única conexão afim $\nabla$ em $M$ que é simétrica e compatível com a métrica g. Tal conexão é chamada conexão de Levi-Civita ou de Riemann. Essa conexão associa a cada par $x, y$ de campos de vetores $C^{\infty}$ em $M$ o campo de vetores $\nabla_{x} y$ chamado de derivada covariante de $y$ na direção $x$. O operador $\nabla_{x} y$ é linear em $x$ mas não em $y$, isto é, $\nabla_{f x+g y} z=f \nabla_{x} z+g \nabla_{y} z$ e $\nabla_{x}(y+z)=\nabla_{x} y+\nabla_{x} z$, mas $\nabla_{x}(f y)=f \nabla_{x} y+x(f) y$, para todos $x, y, z$ campos de vetores $C^{\infty}$ em $M$ (tal conjunto será denotado por $\mathcal{X}(M))$ e $f, g$ funções $C^{\infty}$ em $M$. Além disso, como a conexão de Riemann $\nabla$ em $M$ é compatível com a. métrica, vale que $x<y, z\rangle=\left\langle\nabla_{x} y, z\right\rangle+\left\langle y, \nabla_{x} z\right\rangle$ para todos $x, y$ e $z \in \mathcal{X}(M)$.

No caso de $(G, \mathrm{~g})$ um grupo de Lie com uma métrica invariante à esquerda, temos que $<\nabla_{x} y, z>+\left\langle y, \nabla_{x} z>=0\right.$, para todos $x, y, z \in \mathcal{G}$. Com isso é possível para $x, y, z$ pertencentes à álgebra $\mathcal{G}$ simplificar a fórmula de Koszul:

$$
\begin{gathered}
<\nabla_{x} y, z>=\frac{1}{2}(<[x, y], z>-<[y, z], x>+ \\
<[z, x], y>+x<y, z>-z<x, y>+y<z, x>) \\
=\frac{1}{2}(<[x, y], z>-<[y, z], x>+<[z, x], y>) .
\end{gathered}
$$

$\mathrm{O}$ tensor de curvatura de Riemann $R$ é uma correspondência que associa a cada par $x, y \in$ $\mathcal{X}(M)$ uma aplicaşão $R_{(x, y)}: \mathcal{X}(M) \rightarrow \mathcal{X}(M)$ dada por $R_{x y}=R(x, y)=\nabla_{[x, y]}-\nabla_{x} \nabla_{y}+\nabla_{y} \nabla_{x}$ onde $\nabla$ é a conexão de Riemann de $M$. Dado um ponto $p$ de $M$ e um subespaço bidimensional $\Pi \subset T_{p} M$, o número real $K(x, y)=K(\Pi)$ dado por:

$$
K(\Pi)=K(x, y)=\frac{<R(x, y) x, y>}{\|x\|^{2}\|y\|^{2}-<x, y>^{2}}
$$

é chamado curvatura seccional do plano $\Pi$ em $p$ onde $\{x, y\}$ é uma base para $\Pi$. Sabemos que a curvatura seccional não depende de uma escolha particular de base, dependendo somente do ponto $p \in M$. Pela homogeneidade de um grupo de Lie equipado com métrica invariante à esquerda, podemos olhar para a curvatura seccional somente na identidade do grupo, de maneira que pode ser calculada diretamente na álgebra $\mathcal{G}$. A álgebra por sua vez pode ser descrita através das chamadas constantes estruturais $\alpha_{i j k}$ com relação a uma dada base ortonormal $\left\{e_{1}, \cdots, e_{n}\right\}$, definidas por:

$$
\left[e_{i}, e_{j}\right]=\sum_{k} \alpha_{i j k} e_{k}
$$

(observe que o colchete de Lie de dois campos de vetores invariantes à esquerda é também um campo de vetores invariante à esquerda, logo é combinação linear de elementos da base). 
Temos que $\alpha_{i j k}=<\left[e_{i}, e_{j}\right], e_{k}>$ e é fácil ver que $\alpha_{i j k}=-\alpha_{j i k}$. O lema seguinte diz que podemos expressar a curvatura seccional somente em função das constantes estruturais. O fato importante contido nesse lema é que em um grupo de Lie munido de uma métrica invariante à esquerda, a álgebra de Lie associada e o valor da métrica na identidade determinam completamente a conexão de Riemann.

Lema 1. Com as constantes estruturais $\alpha_{i j k}$ definidas como anteriormente, a curvatura seccional $k\left(e_{1}, e_{2}\right)$ é dada pela fórmula:

$$
\begin{gathered}
k\left(e_{1}, e_{2}\right)=\sum_{k}\left(\frac{1}{2} \alpha_{12 k}\left(-\alpha_{12 k}+\alpha_{2 k 1}+\alpha_{k 12}\right)\right. \\
\left.-\frac{1}{4}\left(\alpha_{12 k}-\alpha_{2 k 1}+\alpha_{k 12}\right)\left(\alpha_{12 k}+\alpha_{2 k 1}-\alpha_{k 12}\right)-\alpha_{k 11} \alpha_{k 22}\right) .
\end{gathered}
$$

Demonstração: Podemos escrever a fórmula de Koszul para os elementos da base ortonormal $\left\{e_{1}, \cdots, e_{n}\right\}$ da álgebra. Assim temos que:

$$
\nabla_{e_{i}} e_{j}=\frac{1}{2} \sum_{k}\left(\alpha_{i j k}-\alpha_{j k i}+\alpha_{k i j}\right) e_{k} .
$$

Agora reescrevamos as fórmulas da curvatura seccional e tensor de Riemann:

$$
\begin{gathered}
k\left(e_{1}, e_{2}\right)=<R\left(e_{1}, e_{2}\right) e_{1}, e_{2}> \\
R\left(e_{1}, e_{2}\right) e_{1}=\nabla_{\left[e_{1}, e_{2}\right]} e_{1}+\nabla_{e_{2}} \nabla_{e_{1}} e_{1}-\nabla_{e_{1}} \nabla_{e_{2}} e_{1} .
\end{gathered}
$$

Substituindo e usando a linearidade do operador $\nabla$, temos:

$$
\begin{gathered}
\nabla_{\left[e_{1}, e_{2}\right]} e_{1}=\nabla_{\sum_{k} \alpha_{12 k} e_{k}} e_{1}=\sum_{k} \alpha_{12 k} \nabla_{e_{k}} e_{1}= \\
\sum_{k} \alpha_{12 k}\left(\frac{1}{2} \sum_{t}\left(\alpha_{k 1 t}-\alpha_{1 t k}+\alpha_{t k 1}\right) e_{t}=\right. \\
=\frac{1}{2} \sum_{k, t} \alpha_{12 k}\left(\alpha_{k 1 t}-\alpha_{1 t k}+\alpha_{t k 1}\right) e_{t} . \\
\nabla_{e_{2}} \nabla_{e_{1}} e_{1}=\nabla_{e_{2}}\left(\frac{1}{2} \sum_{k}\left(\alpha_{11 k}-\alpha_{1 k 1}+\alpha_{k 11}\right) e_{k}\right)= \\
\frac{1}{2} \sum_{k}\left(\alpha_{11 k}-\alpha_{1 k 1}+\alpha_{k 11}\right) \nabla_{e_{2}} e_{k}=
\end{gathered}
$$




$$
\begin{gathered}
\frac{1}{2} \sum_{k}\left(\alpha_{11 k}-\alpha_{1 k 1}+\alpha_{k 11}\right)\left(\frac{1}{2} \sum_{t}\left(\alpha_{2 k t}-\alpha_{k t 2}+\alpha_{t 2 k}\right) e_{t}\right)= \\
\frac{1}{4} \sum_{k, t}\left(\alpha_{11 k}-\alpha_{1 k 1}+\alpha_{k 11}\right)\left(\alpha_{2 k t}-\alpha_{k t 2}+\alpha_{t 2 k}\right) e_{t} . \\
\nabla_{e_{1}} \nabla_{e_{2}} e_{1}=\nabla_{e_{1}}\left(\frac{1}{2} \sum_{k}\left(\alpha_{21 k}-\alpha_{1 k 2}+\alpha_{k 21}\right) e_{k}\right)= \\
\frac{1}{2} \sum_{k}\left(\alpha_{21 k}-\alpha_{1 k 2}+\alpha_{k 21}\right) \nabla_{e_{1}} e_{k}= \\
\frac{1}{2} \sum_{k}\left(\alpha_{21 k}-\alpha_{1 k 2}+\alpha_{k 21}\right)\left(\frac{1}{2} \sum_{t}\left(\alpha_{1 k t}-\alpha_{k t 1}+\alpha_{t 1 k}\right) e_{t}\right)= \\
\frac{1}{4} \sum_{k, t}\left(\alpha_{21 k}-\alpha_{1 k 2}+\alpha_{k 21}\right)\left(\alpha_{1 k t}-\alpha_{k t 1}+\alpha_{t 1 k}\right) e_{t} .
\end{gathered}
$$

Substituindo os valores encontrados em $\left\langle R\left(e_{1}, e_{2}\right) e_{1}, e_{2}>\right.$ e fazendo algumas simplificações, encontra-se a fórmula desejada.

A expressão do lema mostra que a curvatura seccional pode ser obtida conhecendo-se somente a álgebra de Lie e o valor da métrica na identidade, o que simplifica muito seu cálculo. Além disso, a curvatura seccional é um polinômio contínuo em função das constantes $\alpha_{i j k}$ e ela se anula sempre que as constantes se anulam.

Seja $L$ uma transformação linear $L: V \rightarrow V$ onde $V$ é um espaço vetorial de dimensão finita com um produto interno $\langle\cdot, \cdot\rangle$. Existe uma única transformação linear $L^{*}$ de $V$ em $V$ tal que $\langle L(x), y\rangle=\left\langle x, L^{*}(y)\right\rangle$ para todo $x$ e $y$ em $V$, chamada de adjunto de $L$. A transformação $L$ é chamada de anti-adjunta se $L^{*}=-L$. Para cada $x$ pertencente à álgebra $\mathcal{G}$ fixado, a transformação linear $y \mapsto[x, y]$ de $\mathcal{G}$ em $\mathcal{G}$ será denotada por $a d(x)$ ou $a d_{x}$ e será chamada de transformação ou representação adjunta de $\mathcal{G}$. Dado $G$ grupo de Lie equipado com uma métrica invariante à esquerda, seja $u$ um vetor na álgebra de Lie de $G$. Temos o seguinte lema:

Lema 2. Seja u pertencente à álgebra de Lie $\mathcal{G}$ fixado. Se a transformação linear ad(u) for anti-adjunta, então $k(u, v) \geq 0$, para todo $v$ pertencente a $\mathcal{G}$. A igualdade vale se e somente se $u$ é ortogonal ao subespaço $[v, \mathcal{G}]$. 
Demonstração: Sem perda de generalidade, podemos assumir que $u, v$ são ortonormais. Vamos completar $u, v$ a uma base ortonormal $e_{1}, \cdots, e_{n} \operatorname{com} e_{1}=u, e_{2}=v$. Por hipótese $a d^{*}\left(e_{1}\right)=-a d\left(e_{1}\right)$. Logo:

$$
<a d\left(e_{1}\right)\left(e_{i}\right), e_{j}>=-<e_{i}, a d\left(e_{1}\right)\left(e_{j}\right)>
$$

ou seja, $<\left[e_{1}, e_{i}\right], e_{j}>=-<e_{i},\left[e_{1}, e_{j}\right]>$. Segue que $\alpha_{1 i j}=-<e_{i}, \sum_{k} \alpha_{1 j k} e_{k}>=-\alpha_{1 j i}$. Daí extraímos as seguintes relações:

$$
\begin{gathered}
\alpha_{12 k}=-\alpha_{1 k 2}=\alpha_{k 12} \\
\alpha_{k 11}=-\alpha_{1 k 1}=-\left(-\alpha_{11 k}\right)=0 .
\end{gathered}
$$

Temos então que:

$$
\begin{gathered}
k\left(e_{1}, e_{2}\right)=\sum_{k} \frac{1}{2} \alpha_{12 k}\left(\alpha_{2 k 1}\right)-\frac{1}{4}\left(2 \alpha_{12 k}-\alpha_{2 k 1}\right)\left(\alpha_{2 k 1}\right) \\
=\sum_{k} \frac{\left(\alpha_{2 k 1}\right)^{2}}{4} \geq 0
\end{gathered}
$$

e é igual a zero se e somente se $\alpha_{2 k 1}=0$, ou seja, se e somente se $<\left[e_{2}, e_{k}\right], e_{1}>=0$ para todo $k$, logo se e somente se $u$ é ortogonal ao subespaço $[v, \mathcal{G}]$.

Observe que o adjunto de uma transformação, quando existe, depende do produto interno escolhido, ou seja, a hipótese da proposição anterior depende de uma escolha particular de métrica. O próximo corolário livra-nos desta escolha.

Corolário 1. Seja $G$ um grupo de Lie. Se u pertence ao centro da álgebra de Lie $\mathcal{G}$ de $G$, então para qualquer métrica invariante à esquerda, a desigualdade $k(u, v) \geq 0$ é satisfeita para todo $v \in \mathcal{G}$.

Demonstração: Se $u$ pertence ao centro de $\mathcal{G}$, então $[u, y]=0$, para todo $y$ em $\mathcal{G}$. Logo a transformação adjunta $a d(u)$ é nula. Como a transformação nula é sempre anti-adjunta, segue da proposição anterior que $k(u, v) \geq 0$, para todo $v$ em $\mathcal{G}$.

Para os próximos resultados, lembremos a definição de aplicação exponencial. Seja $X$ um campo de vetores invariante à esquerda. Sabemos como consequência do teorema de existência e unicidade de equações diferenciais que existe $\epsilon>0$ e uma curva integral $\phi_{X}:(-\epsilon, \epsilon) \rightarrow G$ $\operatorname{com} \phi_{X}(0)=1$. Não é difícil verificar que o campo $X$ é completo, ou seja, suas curvas integrais estão definidas em todo $\mathbb{R}$. A aplicação exponencial de $G$ é definida como sendo a aplicação 
$\exp : \mathcal{G} \rightarrow G$ que leva $X$ em $\phi_{X}(1)$. Observe que $\left.\frac{d}{d t}\right|_{t=0} \phi_{X}(s t)=s \phi_{X}^{\prime}(0)=s X$ e $\phi_{s X}(t)=\phi_{X}(s t)$. Em particular $\phi_{X}(t)=\phi_{t X}=\exp (t X)$ e assim $\left.\frac{d}{d t}\right|_{t=0} \exp (t X)=\left.\frac{d}{d t}\right|_{t=0} \phi_{X}(t)=X$. Também não é difícil concluir que a aplicação exponencial é diferenciável e sua diferencial na origem é a identidade.

Temos também a exponencial definida na variedade Riemanniana $G$. Seja $\Omega \subset T G$ (onde $T G$ é o fibrado tangente de $G$ ) definido por $\Omega=\left\{v \in T G: \gamma_{v}(1)\right.$ está definida $\}$ onde $\gamma_{v}$ é a geodésica com $\gamma_{v}^{\prime}(0)=v \in T_{p} G, \gamma_{v}(0)=p \in G$. É possível mostrar que $\Omega$ é aberto de $T G$. A aplicação exponencial, $\exp _{p}: \Omega \subset T G \rightarrow G$ em $p \in G$ é definida por $\exp _{p}(v)=\gamma_{v}(1)$. Temos que a. aplicação exponencial é diferenciável e, além disso, dado $p \in G$, a aplicação $\Phi: \Omega \rightarrow G \times G$ definida por $\Phi(v)=\left(\pi(v), \exp _{\pi(v)} v\right)$ é um difeomorfismo local de uma vizinhança de $0_{p}$ em $T M$ sobre uma vizinhança de $(p, p)$ em $G \times G$, onde $\pi: T G \rightarrow G$ é a projeção $\pi^{-1}(p)=T_{p} G$. Como consequência, dado $p \in G$, existe uma vizinhança $U$ de $p$ em $G$ e um $\epsilon>0$ tais que $\exp _{p}$ é um difeomorfismo entre a bola $B_{O_{p}}(\epsilon) \subset T_{p} G$ e sua imagem em $G$. Em geral as exponenciais do grupo e Riemanniana não coincidem, mas se o grupo $G$ é equipado com uma métrica biinvariante, isto é, invariante à esquerda e à direita; observe que nesse caso as geodésicas de $G$ são curvas integrais de campos invariantes à esquerda.

Precisamos também da definição de representação adjunta de um grupo de Lie $G$. Seja $g \in G$; podemos definir um automorfismo de $G, \operatorname{Inn}(g): G \rightarrow G, \operatorname{Inn}(g)(x)=g x g^{-1}$. Essa aplicação é de fato um automorfismo de $G$, chamado automorfismo interno definido por $g$. A diferencial $d(\operatorname{Inn}(g)): \mathcal{G} \rightarrow \mathcal{G}$ define um automorfismo de $\mathcal{G}$, que denotamos por $A d(g)$. Temos então:

$$
\operatorname{Ad}(g)(X)=\left.\frac{d}{d t}\right|_{t=0} \operatorname{Inn}(g)(\exp t X)=\left.\frac{d}{d t}\right|_{t=0} g \exp t X g^{-1}
$$

O homomorfismo $A d: g \in G \rightarrow A d(g) \in A u t(\mathcal{G})$ é chamado representação adjunta de $G$. A diferencial $d(A d)$ it define a representação adjunta de $\mathcal{G}$ em $\mathcal{G}$ que é denotada por ad. É possível mostrar que esta definição e a dada anteriormente usando o colchete coincidem, veja [23].

O seguinte resultado envolve métricas bi-invariantes e a aplicação $\operatorname{Ad}(g)$.

Proposição 1. Seja $G$ um grupo de Lie. Então $G$ possuir uma métrica bi-invariante é equivalente a existir um produto interno em $T_{e} G \simeq \mathcal{G}$ Ad-invariante, isto é, tal que

$$
<A d_{g}(u), A d_{g}(v)>=<u, v>
$$

para todos $u, v$ pertencentes a $\mathcal{G}$. Isto implica em a transformação adjunta ser anti-adjunta $e$ vale a recíproca se $G$ é conexo.

Demonstração: Suponhamos que $G$ possui uma métrica bi-invariante. Então vamos re- 
stringir essa métrica ao espaço tangente à identidade. Temos, para todo $g \in G$ e $u, v \in T_{e} G$ :

$$
\begin{gathered}
<A d_{g}(x), A d_{g}(y)>_{e}=<d\left(L_{g} R_{g^{-1}}\right)_{e}(x), d\left(L_{g} R_{g^{-1}}\right)_{e}(y)>_{e} \\
=<\left(d L_{g}\right)_{g^{-1}}\left(d R_{g^{-1}}\right)_{e}(x),\left(d L_{g}\right)_{g^{-1}}\left(d R_{g^{-1}}\right)_{e}(y)>_{e} \\
=<\left(d R_{g^{-1}}\right)_{e}(x),\left(d R_{g^{-1}}\right)_{e}(y)>_{g^{-1}}=<x, y>_{e} .
\end{gathered}
$$

Reciprocamente, suponhamos que existe um produto interno $\langle,, \cdot\rangle_{e}$ em $\mathcal{G}$ que é Adinvariante. Podemos estender esse produto interno a uma métrica invariante à esquerda fazendo $\left.\langle u, v\rangle_{g}=<\left(d L_{g^{-1}}\right)_{g}(u),\left(d L_{g^{-1}}\right)_{g}(v)\right\rangle_{e}$ para todos $u, v \in T_{g} G$ e $g \in G$. Vamos mostrar que essa métrica (que é evidentemente invariante à esquerda) também é invariante à direita. De fato:

$$
\begin{gathered}
<\left(d R_{h}\right)_{g}(u),\left(d R_{h}\right)_{g}(v)>_{R_{h}(g)} \\
=<\left(d L_{h^{-1}} g^{-1}\right)_{g h}\left(d R_{h}\right)_{g}(u),\left(d L_{h^{-1}} g^{-1}\right)_{g h}\left(d R_{h}\right)_{g}(v)>_{e} \\
=<d\left(L_{h^{-1}} L_{g^{-1}}\right)_{g h}\left(d R_{h}\right)_{g}(u), d\left(L_{h^{-1}} L_{g^{-1}}\right)_{g h}\left(d R_{h}\right)_{g}(v)>_{e} \\
=<\left(d L_{h^{-1}}\right)_{h}\left(d L_{g^{-1}}\right)_{g h}\left(d R_{h}\right)_{g}(u),\left(d L_{h^{-1}}\right)_{h}\left(d L_{g^{-1}}\right)_{g h}\left(d R_{h}\right)_{g}(v)>_{e} \\
=<\left(d L_{h^{-1}}\right)_{h} d\left(L_{g^{-1}} R_{h}\right)_{g}(u),\left(d L_{h^{-1}}\right)_{h} d\left(L_{g^{-1}} R_{h}\right)_{g}(v)>_{e} \\
=<\left(d L_{h^{-1}}\right)_{h} d\left(R_{h} L_{g^{-1}}\right)_{g}(u),\left(d L_{h^{-1}}\right)_{h} d\left(R_{h} L_{g^{-1}}\right)_{g}(v)>_{e} \\
=<\left(d L_{h^{-1}}\right)_{h}\left(d R_{h}\right)_{e}\left(d L_{g^{-1}}\right)_{g}(u),\left(d L_{h^{-1}}\right)_{h}\left(d R_{h}\right)_{e}\left(d L_{g^{-1}}\right)_{g}(v)>_{e} \\
=<d\left(L_{h^{-1}} R_{h}\right)_{e}\left(d L_{g^{-1}}\right)_{g}(u), d\left(L_{h^{-1}} R_{h}\right)_{e}\left(d L_{g^{-1}}\right)_{g}(v)>_{e} \\
=<A d_{h}\left(\left(d L_{g^{-1}}\right)_{g}(u)\right), A d_{h}\left(\left(d L_{g^{-1}}\right)_{g}(v)\right)>_{e} \\
=<\left(d L_{g^{-1}}\right)_{g}(u)_{1}\left(d L_{g^{-1}}\right)_{g}(v)>_{e}=<u, v>_{g} .
\end{gathered}
$$

Portanto, vale a recíproca. Agora suponhamos que existe um produto interno em $\mathcal{G}$ que é $A d$-invariante. Vamos mostrar que $a d_{x}^{*}=-a d_{x}$, para todo $x \in \mathcal{G}$. Temos por hipótese que $<A d_{g}(x), A_{g}(y)>=<x, y>$, para todo $x, y \in \mathcal{G}$ e para todo $g \in G$. Seja $g=\exp t z$, para $z \in \mathcal{G}$. Portanto $<A d_{\exp t z}(x), A d_{\exp t z}(y)>=<x, y>$. Logo:

$$
\begin{gathered}
\left.\frac{d}{d t}\right|_{t=0}<A d_{\exp t z}(x), A d_{\exp t z}(y)>=0 \Rightarrow \\
<\left.\frac{d}{d t}\right|_{t=0} A d_{\exp t z}(x),\left.A d_{\exp t z}(y)\right|_{t=0}>+<\left.A d_{\exp t z}(x)\right|_{t=0},\left.\frac{d}{d t}\right|_{t=0} A d_{\exp t z}(y)>=0 \Rightarrow \\
<\left.\frac{d}{d t}\right|_{t=0} \exp \left(\left(t a d_{z}\right)(x)\right), y>+<x,\left.\frac{d}{d t}\right|_{t=0} \exp \left(\left(\operatorname{tad}_{z}\right)(y)\right)>=0
\end{gathered}
$$


e segue que $\left\langle a d_{z}(x), y\right\rangle+\left\langle x, a d_{z}(y)\right\rangle=0$ o que mostra que a aplicação $a d_{z}$ é anti-adjunta para todo $z \in \mathcal{G}$. A volta vale para todas as passagens exceto a primeira. Gostaríamos de mostrar que:

$$
\left.\frac{d}{d t}\right|_{t=0}<A d_{\exp t z}(x), A d_{\exp t z}(y)>=0 \Rightarrow<A d_{\exp t z}(x), A d_{\exp t z}(y)>=<x, y>.
$$

Então fixemos $x, y, z$ pertencentes à $\mathcal{G}$. Seja $f_{x y z}: \mathbb{R} \rightarrow \mathbb{R}$ dada por

$$
f_{x y z}(t)=<A d_{\exp t z}(x), A d_{\exp t z}(y)>\text {. }
$$

Temos que $f_{x y z}(0)=<x, y>$ e $f_{x y z}^{\prime}(0)=0$. Agora observemos que:

$$
\begin{aligned}
& f_{x y z}^{\prime}(t)=\left.\frac{d}{d h}\right|_{h=0} f_{x y z}(t+h) \\
& =\left.\frac{d}{d h}\right|_{h=0}<A d_{\exp _{(t+h) z}}(x), A d_{\exp _{(t+h) z}}(y)>
\end{aligned}
$$

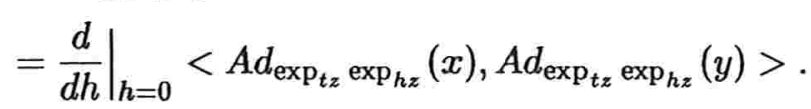

Chamemos $\tilde{x}=A d_{\exp _{h z}}(x)$ e $\tilde{y}=A d_{\exp _{h z}}(y)$. Então $f_{x y z}^{\prime}(t)=\left.\frac{d}{d h}\right|_{h=0} f_{\tilde{x} \tilde{y} z}(h)=0$. Logo $f_{x y z}(t)$ é constante, do que segue que $f_{x y z}(t)=\langle x, y\rangle$ para todos $x, y$, $z$ pertencentes a $\mathcal{G}$. Mostramos que $\left\langle A d_{g}(x), A d_{g}(y)>=<x, y>\right.$ para todos $x, y$ pertencentes a $\mathcal{G}$ e $g$ em uma vizinhança da identidade. Como $G$ é gerado por uma vizinhança da identidade, sendo $G$ conexo segue que $<\operatorname{Ad}_{g}(x), \operatorname{Ad}_{g}(y)>=<x, y>$ para todo $g \in G$.

Usando esta. proposição, podemos mostrar que um grupo de Lie admite uma métrica biinvariante construindo um produto interno $A d$-invariante na sua álgebra. Por exemplo, veremos na Seção 8 que se $G$ é compacto, conseguimos construir tal produto interno. Mais geralmente, mostraremos que um grupo de Lie admite uma métrica bi-invariante se e somente se ele é isomorfo a um produto cartesiano de um grupo de Lie compacto com um grupo vetorial aditivo (um $\mathbb{R}^{n}$ ). Na realidade esta proposição vale para um caso mais geral. Se $V$ é um espaço vetorial real (ou complexo), dizemos que a representação de $G$ em $V \rho: G \rightarrow A u t(V)$ é ortogonal (unitária) se $<\rho(g)(u), \rho(g)(v)>=<u, v>$ para todo $g \in G$ e $u, v \in V$. Então dada uma representação $\rho$ de um grupo de Lie compacto sobre um espaço vetorial real (complexo), existe um produto interno em $V$ sobre o qual $\rho$ é ortogonal (unitário).

Em um grupo de Lie com uma métrica bi-invariante, todas as curvaturas seccionais são maiores ou iguais a zero, melhor dizendo, a curvatura seccional satisfaz:

$$
k(x, y)=\frac{1}{4}<[x, y],[x, y]>
$$


De fato, se $G$ possui métrica bi-invariante, pela proposição anterior, $a d_{x}$ é anti-adjunta, para qualquer $x \in \mathcal{G}$. Logo:

$$
\begin{gathered}
<[z, x], y>=<a d_{z} x, y>=-<x, a d_{z} y> \\
=-<x,[z, y]>=<[y, z], x>.
\end{gathered}
$$

Usando a fórmula de Koszul, temos que $\left\langle\nabla_{x} y, z\right\rangle=\frac{1}{2}\langle[x, y], z\rangle$. Logo, $\nabla_{x}=\frac{1}{2} a d_{x}$. Substituindo no tensor de Riemann:

$$
\begin{gathered}
R_{x y}=\nabla_{[x, y]}-\nabla_{x} \nabla_{y}+\nabla_{y} \nabla_{x} \\
=\frac{1}{2} a d_{[x, y]}-\frac{1}{4} a d_{x} a d_{y}+\frac{1}{4} a d_{y} a d_{x} .
\end{gathered}
$$

Usando agora a fórmula de Jacobi, segue que $a d_{[x, y]}-a d_{x} a d_{y}+a d_{y} a d_{x}=0$. Logo, $R_{x y}=\frac{1}{4} a d_{[x, y]}$. Finalmente substituindo na fórmula da curvatura seccional, segue:

$$
\begin{gathered}
k(x, y)=<R_{x y} x, y>=\frac{1}{4}\left\langle a d_{[x, y]}(x), y>\right. \\
\left.=\frac{1}{4}<[[x, y], x], y\right\rangle=-\frac{1}{4}<[x,[x, y]], y> \\
=-\frac{1}{4}\left\langle a d_{x}([x, y]), y>=\frac{1}{4}\langle[x, y],[x, y]\rangle\right.
\end{gathered}
$$

É interessante observar que os casos de curvatura seccional estritamente positiva são raros. Um teorema de Wallach (veja [22]) que demonstraremos na Seção 10 diz que $S U(2)$ (matrizes $2 \times 2$ complexas unitárias com determinante igual a 1) é o único grupo de Lie simplesmente conexo que admite uma métrica invariante à esquerda com curvatura seccional estritamente positiva. Para o caso de curvatura seccional identicamente nula observamos que se a álgebra $\mathcal{G}$ é comutativa, então o colchete é identicamente nulo, dessa maneira as constantes estruturais são nulas, o que implica que para qualquer métrica invariante à esquerda a curvatura seccional é identicamente nula, pelo Lema 1. A recíproca não vale sempre, veremos na Seçao 6 que o grupo $E(2)$ constituído pelos movimentos rígidos do plano euclideano é um grupo de Lie não comutativo que possui métrica invariante à esquerda flat. Na Seção 9 classificaremos os grupos de Lie com métrica invariante à esquerda flat, onde mostraremos que a. caracterização nesse caso é através da álgebra.

O caso de curvatura seccional menor ou igual a zero foi classificado por R.Azencott e E.Wilson, veja [2]. Um exemplo deste caso é interessante a ser estudado. Antes precisamos da seguinte: 
Proposição 2. Seja $G$ um grupo de Lie com uma métrica invariante à esquerda $<\cdot, \cdot\rangle$. Suponhamos que a álgebra de Lie de $G, \mathcal{G}$, contenha um ideal $\mathcal{U}$ de codimensão 1 . Seja $b$ um vetor ortogonal a $\mathcal{U}$. Seja $L: \mathcal{U} \rightarrow \mathcal{U}$ a transformação ad(b) restrita a $\mathcal{U}$, ou seja, $L(u)=[b, u]$, para $u \in \mathcal{U}$. Seja $L^{*}$ o adjunto de $L$ e seja $S=\frac{1}{2}\left(L+L^{*}\right)$ a parte auto-adjunta de $L$. Então o operador derivada covariante $\nabla_{b}$ satisfaz $\nabla_{b} b=0$ e $\nabla_{b} u=\frac{1}{2}\left(L-L^{*}\right) u$, para cada $u \in \mathcal{U}$. Além disso, o operador $\nabla_{u}$ satisfaz $\nabla_{u} b=-S u$ e $\nabla_{u} v=\bar{\nabla}_{u} v+\langle S u, v\rangle b$, para todo $u$ e $v$ pertencentes a $\mathcal{U}$, onde $\bar{\nabla}$ é a conexão de Riemann para a subálgebra $\mathcal{U}$, onde $\mathcal{U}$ possui a métrica induzida de $\mathcal{G}$.

Demonstração: Usando a fórmula de Koszul:

$$
<\nabla_{x} y, z>=\frac{1}{2}(<[x, y], z>+<[z, x], y>-<[y, z], x>),
$$

temos que $\left\langle\nabla_{b} b, b\right\rangle=0$. Além disso, para todo $w \in \mathcal{U}$ :

$$
<\nabla_{b} b, w>=\frac{1}{2}(<[b, b], w>-<[b, w], b>+<[w, b], b>)
$$

a primeira parcela é igual a zero pois $[b, b]=0$, a segunda também é zero pois $[b, w] \in \mathcal{U}$ já que $\mathcal{U}$ é um ideal e $b$ é ortogonal a $\mathcal{U}$ e, pelo mesmo motivo, a terceira parcela também é zero. Logo $\nabla_{b} b=0$. Analogamente,

$$
\begin{aligned}
<\nabla_{b} u, w>=\frac{1}{2}(<[b, u], w>+<[w, b], u>)=\frac{1}{2}(<[b, u], w>-<u,[b, w]>) \\
=\frac{1}{2}\left(<L(u), w>-<L^{*}(u), w>\right)=\frac{1}{2}<\left(L-L^{*}\right)(u), w>,
\end{aligned}
$$

para qualquer $w$ em $\mathcal{U}$, em particular para $w=\left(L-L^{*}\right)(u)$. Portanto, $\nabla_{b} u=\frac{1}{2}\left(L-L^{*}\right)(u)$, para todo $u \in \mathcal{U}$. Temos também que:

$$
\begin{aligned}
<\nabla_{u} b, w>= & \frac{1}{2}(<[u, b], w>-<[b, w], u>)=\frac{1}{2}(<-L(u), w>+<-L(w), u>) \\
& =\frac{1}{2}\left(<-L(u), w>+<-L^{*}(u), w>\right)=<-S(u), w>,
\end{aligned}
$$

para todo $w \in \mathcal{U}$. Logo, $\nabla_{u} b=-S(u)$, para todo $u \in \mathcal{U}$.

Para concluir a demonstração, queremos mostrar que $\nabla_{u} v=\bar{\nabla}_{u} v+\langle S u, v\rangle b$. Vamos calcular a componente na direção $b$ :

$$
\begin{gathered}
\left\langle\nabla_{u} v, b>=\frac{1}{2}(-<[v, b], u>+<[b, u], v>)\right. \\
=\frac{1}{2}\left(<L^{*}(v), u>+\langle L(u), v>)=\frac{1}{2}\left(\left\langle\left(L+L^{*}\right)(u), v>\right) .\right.\right.
\end{gathered}
$$

Logo, $\left\langle\nabla_{u} v, b\right\rangle=\langle S(u), v\rangle$. Agora calculemos a componente ortogonal a $b$. Seja $w$ pertencente a. $\mathcal{U}$ qualquer:

$$
<\nabla_{u} v, w>=\frac{1}{2}(<[u, v], w>-<[v, w], u>+<[w, u], v>)
$$


Sendo $u, v, w$ pertencentes à $\mathcal{U}$, temos que $\left\langle\nabla_{u} v, w\right\rangle=\left\langle\bar{\nabla}_{u} v, w\right\rangle$. Portanto, $\nabla_{u} v=\bar{\nabla}_{u} v+<$ $S u, v>b$. Isto termina a demonstração.

O seguinte exemplo é interessante:

\subsection{Exemplo:}

Seja $G$ um grupo de Lie com métrica invariante à esquerda $g$ e seja $\mathcal{G}$ a álgebra de Lie de $G$ com dimensão maior ou igual a 2. Suponhamos que o colchete $[x, y]$ é uma combinação linear de $x$ e $y$, isto é, $[x, y]=\alpha x+\beta y$ onde a princípio $\alpha$ e $\beta$ dependem de $x$ e $y$. Queremos mostrar que $[x, y]=l(y) x+l(x) y$, onde $l: \mathcal{G} \rightarrow R$ é um funcional linear. Queremos concluir finalmente que a curvatura seccional é constante e estritamente negativa.

Seja $x \in \mathcal{G}$ fixado. Seja $\mathbb{R} x=\{a x: a \in \mathbb{R}\}$ um subespaço vetorial de $\mathcal{G}$ e considere o quociente $\mathcal{G} / \mathbb{R} x=\{\bar{v}: v \in \mathcal{G}\}$ onde $\bar{v}=\{u \in \mathcal{G}: u \sim v\}=\{u \in \mathcal{G}: u-v \in \mathbb{R} x\}$. Seja $\pi: \mathcal{G} \rightarrow \mathcal{G} / \mathbb{R} x$ a projeção $\pi(y)=\bar{y}=\{u \in \mathcal{G}: u-y=k x, k \in \mathbb{R}\}$. Como $\mathbb{R} x \subset \operatorname{ker}\left(a d_{x}\right)$, a aplicação ajunta $a d_{x}: \mathcal{G} \rightarrow \mathcal{G}$ induz uma aplicação bem definida de $\mathcal{G} / \mathbb{R} x$ em $\mathcal{G} / \mathbb{R} x$ denotada por $\overline{a d}_{x}$, e dada por $\overline{a d}_{x}(\bar{y})=\overline{[x, y]}$. Temos que $\overline{[x, y]}=\alpha \bar{x}+\beta \bar{y}=\alpha \overline{0}+\beta \bar{y}=\beta \bar{y}$. Como $\overline{a d}_{x}$ é linear, $\beta$ não depende de $y, \operatorname{logo} \beta=l(x)$, para $l: \mathcal{G} \rightarrow \mathbb{R}$ uma função que não sabemos ser linear. Portanto $\overline{[x, y]}=\overline{a d}_{x}(\bar{y})=l(x) \bar{y}$. Observe que $[x, y] \in \overline{[x, y]}$. Logo $[x, y] \in l(x) \bar{y}$, segue que $[x, y]-l(x) y=k x$ onde $k$ pertence a $\mathbb{R}$, ou seja, $[x, y] \equiv l(x)(y) \bmod \mathbb{R} x$. Seja $\left\{x, y_{1}, \cdots, y_{n-1}\right\}$ uma base de $\mathcal{G}$. Podemos escrever a matriz de $\operatorname{ad}(x)$ nesta base:

$$
\begin{gathered}
a d_{x}(x)=0 \\
a d_{x}\left(y_{1}\right)=\left[x, y_{1}\right] \equiv l(x) y_{1} \quad \bmod \mathbb{R} x \\
\vdots \\
a d_{x}\left(y_{n-1}\right) \equiv l(x) y_{n-1} \quad \bmod \mathbb{R} x .
\end{gathered}
$$

Segue que o traço de $a d_{x}$ nesta base satisfaz $\operatorname{tr}\left(a d_{x}\right)=(n-1) l(x)$. Portanto, $l$ é linear em $x$. Podemos trocar $x$ por $y$ no início do exemplo e analogamente encontramos que $[x, y]+l(y) x=c y$ onde $c \in \mathbb{R}$. Suponhamos que $x, y$ são linearmente independentes. Anteriormente tínhamos $[x, y]-l(x) y=k x$ com $k \in \mathbb{R}$. Juntando as duas equações e usando que $x$ e $y$ são l.i. segue que $[x, y]=l(x) y-l(y) x$. Supondo $x, y$ linearmente dependentes, segue a mesma fórmula.

Queremos agora achar a curvatura seccional onde supomos que $l$ é um funcional não nulo. Seja $\mathcal{U}$ o núcleo do operador $l$, que é um ideal comutativo, pois se $x \in \mathcal{G}$ e $y \in \mathcal{U}$ então 
$[x, y]=l(x) y-l(y) x=l(x) y \in \mathcal{U}$. Escolhamos um vetor unitário $b$ ortogonal a $\mathcal{U}$ e seja $\lambda=l(b)$. Usando as notações da Proposição 2, temos que $L(u)=[b, u]=l(b) u-l(u) b=l(b) u$, pois $u$ pertence ao kernel de $l$. Logo $L(u)=\lambda u$. Aplicando a proposição 2 , segue que $\nabla_{b}$ é identicamente nulo, pois $L=L^{*}$. Seja $z$ pertencente a $\mathcal{G}$, que podemos escrever como $u+k b$, onde $k$ é uma constante real e $u \in \mathcal{U}$. Queremos calcular $\nabla_{u} z$. Substituindo, $\nabla_{u} z=\nabla_{u}(u+k b)=\nabla_{u} u+k \nabla_{u} b$. De acordo com a proposição $2, \nabla_{u} u=\bar{\nabla}_{u} u+<S u, u>b$. Mas $\bar{\nabla}_{u} u=0$ (pois $\mathcal{U}$ é comutativo) e $k \nabla_{u} b=-k S u$. Logo $\nabla_{u} z=0+<S u, u>b-k S u$. Como $k=<z, b>$, temos que $\nabla_{u} z=<S u, u>b-<z, b>S u$. Usando que $S u=\lambda u$, temos que $\nabla_{u} z=\lambda(<u, u>b-<$ $z, b>u)=\lambda(<u, z>b-<z, b>u)$.

Com isso podemos mostrar que o tensor de Riemann é dado por:

$$
R_{x y}(z)=\lambda^{2}(x<y, z>-y<x, z>)
$$

para quaisquer $x, y, z$ pertencentes a $\mathcal{G}$. Se $x=b, y=u \in \mathcal{U}$ então $\nabla_{[b, u]}=\lambda \nabla_{u}$ e como $\nabla_{b} \equiv 0$, temos $R_{b u}=\nabla_{[b, u]}-\nabla_{b} \nabla_{u}+\nabla_{u} \nabla_{b}=\lambda \nabla_{u}$. Este resultado coincide com a fórmula desejada.

Agora suponhamos que $x, y$ pertençam a $\mathcal{U}$ e seja $z \in \mathcal{G}$ qualquer. Temos que $R_{x y}(z)=$ $\nabla_{[x, y]} z-\nabla_{x} \nabla_{y} z+\nabla_{y} \nabla_{x} z$ e o colchete $[x, y]$ é dado por $l(x) y-l(y) x$. Como por hipótese $x$ e $y$ pertencem ao kernel de $l$, o colchete é nulo. Logo $\nabla_{[x, y]} z$ é nulo. Além disso:

$$
\nabla_{x} \nabla_{y} z=\lambda\left(b<x, \nabla_{y} z>-x<b, \nabla_{y} z>\right)
$$

e o colchete $[x, y]$ é nulo, $[y, z]=l(y) z-l(z) y=-l(z) y$ e $[z, x]=l(z) x$. Substituindo na fórmula de Koszul:

$$
\begin{gathered}
<x, \nabla_{y} z>=\frac{1}{2}(-<l(z) y, x>-<l(z) x, y>)=-l(z)<x, y> \\
<b, \nabla_{y} z>=\frac{1}{2}(0+\lambda<y, z>-<[z, b], y>)=\lambda<z, y>.
\end{gathered}
$$

Logo:

$$
\nabla_{x} \nabla_{y} z=-\lambda l(z)<x, y>b-\lambda^{2}<z, y>x
$$

Analogamente:

$$
\begin{gathered}
\nabla_{y} \nabla_{x} z=\lambda\left(b<y, \nabla_{x} z>-y<b, \nabla_{x} z>\right), \\
<y, \nabla_{x} z>=-l(z)<x, y>, \\
<b, \nabla_{x} z>=\lambda<z, x>, \\
\nabla_{y} \nabla_{x} z=\lambda(-l(z))<x, y>b-\lambda^{2}<z, x>y .
\end{gathered}
$$

Substituindo na fórmula do tensor de Riemann conquistamos exatamente a fórmula $R_{x y}(z)=$ $\lambda^{2}(x<y, z>-y<x, z>)$. A fórmula para $x, y \in \mathcal{G}$ é obtida considerando a bilinearidade de $R_{x y}$. 
Lembremos que a curvatura seccional é dada por $k(x, y)=<R_{x y}(x), y>$, para $x, y$ unitários. Substituindo, temos:

$$
k(x, y)=\lambda^{2}\left(<x, y>^{2}-<x, x><y, y>\right) .
$$

Portanto, a curvatura seccional é igual a $-\lambda^{2}$, quando $x, y$ são dois vetores quaisquer ortonormais na álgebra, o que conclui o exemplo.

Veremos na próxima seção (Teorema 3) que estes exemplos que encontramos são raros. Outros exemplos de curvatura estritamente negativa serão estudados na seção 6. Em 1974, E. Heintze [9] classificou as métricas invariantes à esquerda com curvatura seccional estritamente negativa. Ele mostrou que nesse caso a álgebra de Lie se decompõe em uma soma direta $[\mathcal{G}, \mathcal{G}] \oplus$ $\mathbb{R} x$ para algum $x \in \mathcal{G}$ tal que todos os autovalores de $a d(x)$ restrita a $[\mathcal{G}, \mathcal{G}]$ possuem parte real estritamente positiva. 


\section{Curvatura de Ricci}

Seja $x=e_{n}$ um vetor unitário em $T_{p} G$ e $\left\{e_{1}, \cdots, e_{n-1}\right\}$ base do hiperplano de $T_{p} G$ ortogonal a. $x$. Considere:

$$
\operatorname{Ric}_{p}(x)=\frac{1}{n-1} \sum_{i=1}^{n-1}<R\left(x, e_{i}\right) x, e_{i}>
$$

Sejam $u, v$ pertencentes a $T_{p} G$. Façamos $Q(x, y)=$ traço da aplicação $z \mapsto R(x, z) y . Q$ é bilinear pois o tensor de Riemann é bilinear. Seja $\left\{e_{1}, e_{2}, \cdots, e_{n-1}, e_{n}=x\right\}$ uma base ortonormal de $T_{p} G$. Temos:

$$
Q(x, y)=\sum_{i=1}^{n}<R\left(x, e_{i}\right) y, e_{i}>=\sum_{i=1}^{n}<R\left(y, e_{i}\right) x, e_{i}>=Q(y, x)
$$

onde a segunda igualdade é uma propriedade do tensor de Riemann. Logo $Q$ é bilinear simétrica. Agora:

$$
Q(x, x)=\sum_{i=1}^{n}<R\left(x, e_{i}\right) x, e_{i}>=\sum_{i=1}^{n-1}<R\left(x, e_{i}\right) x, e_{i}>=(n-1) R i c_{p}(x) .
$$

Seja $r(x)=Q(x, x)$. Se $x$ é um vetor unitário, chamamos $r(x)$ de curvatura de Ricci na direção $x$. A curvatura de Ricci na direção $x$ é soma de curvaturas seccionais de planos gerados por $x$ e elementos de uma base ortonormal. Podemos olhar isso também como a contração do tensor de curvatura.

Para alguns cálculos, pode ser mais conveniente trabalhar com a chamada transformação de Ricci auto-adjunta, definida por $\widehat{r}(x)=\sum_{i} R_{e_{i} x}\left(e_{i}\right)$ e que está relacionada com a forma quadrática $r$ pela identidade $r(x)=<\widehat{r}(x), x>$. Os autovalores de $\widehat{r}$ são chamados de curvaturas principais de Ricci. Seja $\left\{e_{1}, \cdots, e_{n}\right\}$ uma base ortonormal de $T_{p} G$ consistindo de autovetores de $\widehat{r}$. Dessa maneira $r$ é diagonalizável e $r\left(\xi_{1} e_{1}+\cdots+\xi_{n} e_{n}\right)=\sum_{i} r\left(e_{i}\right) \xi_{i}^{2}$ onde, na realidade, cada $r\left(e_{i}\right)$ é uma curvatura principal de Ricci.

No caso de um grupo de Lie equipado com métrica invariante à esquerda, a curvatura de Ricci, assim como a curvatura seccional, pode ser calculada somente na álgebra de Lie associada. É intuitivo pensar então que teremos propriedades parecidas com aquelas mostradas na seção anterior. De fato, temos o seguinte lema:

Lema 3. Seja $G$ um grupo de Lie munido de uma métrica invariante à esquerda e seja u vetor unitário pertencente à álgebra associada $\mathcal{G}$. Se a transformação linear ad(u) é anti-adjunta, então $r(u) \geq 0$ onde a igualdade vale se e somente se u é ortogonal a $[\mathcal{G}, \mathcal{G}]$. 
Demonstração: Usando a notação anterior, temos que:

$$
r(u)=\sum_{i=1}^{n}<R\left(u, e_{i}\right) u, e_{i}>=\sum_{i=1}^{n} k\left(u, e_{i}\right) .
$$

Pelo Lema 2 da seção anterior, se $a d(u)$ é anti-adunta, $k(u, v) \geq 0$ para todo $v$ em $\mathcal{G}$. Logo $r(u) \geq 0$ e $r(u)$ é igual a zero se e somente se $k\left(u, e_{i}\right)=0$, para todo $i$, ou seja, se e somente se $u$ é ortogonal ao subespaço $\left[e_{i}, \mathcal{G}\right]$ para todo $i$, pelo Lema 2. Isto termina a demonstração.

Vimos também na seção anterior que se $u$ pertence ao centro da álgebra $\mathcal{G}$, então a aplicação $a d(u)$ é identicamente nula e portanto é anti-adjunta. Logo, se $u$ pertence ao centro de $\mathcal{G}$, $r(u) \geq 0$. Resultados mais interessantes aparecem agora, o que é de se esperar já que a contração do tensor de curvatura simplifica muito seu cálculo. Agora surge a primeira relação entre uma propriedade topológica do grupo com uma propriedade de curvatura. O próximo teorema é uma espécie de recíproca Teorema de Bonnet-Myers:

Teorema 1. Um grupo de Lie conexo admite uma métrica invariante à esquerda com todas as curvaturas de Ricci estritamente positivas se e somente se ele é compacto e possui grupo fundamental finito.

Demonstração: Suponhamos que $G$ admite uma métrica invariante à esquerda com todas as curvaturas de Ricci estritamente positivas. Precisamos garantir que neste caso as curvaturas de Ricci não se aproximam de zero, isto é, $r(x) \geq \delta>0$, para todo $x$ e para algum $\delta>0$. Com isto podemos usar o teorema de Bonnet-Myers. Este teorema diz exatamente que se a curvatura de Ricci satisfaz tal desigualdade, então a variedade é compacta e possui grupo fundamental finito. Considere a transformação de Ricci auto-adjunta $\hat{r}$ caracterizada por $r(x)=\langle\hat{r}(x), x>$. Existe uma base ortonormal $\left\{e_{1}, \cdots, e_{n}\right\}$ em $\mathcal{G}$ formada por autovetores de $\hat{r}$. Logo, $\hat{r}\left(e_{i}\right)=\lambda_{i} e_{i}$ e $r\left(e_{i}\right)=\lambda_{i}$. Como a variedade é homogênea, os autovalores são constantes (por isso estamos olhando somente para a identidade) e são estritamente positivos, por hipótese. Peguemos o menor deles, e chamemos de $M$. Qualquer $x$ na álgebra é dado por $x=\sum_{i} c_{i} e_{i}$, onde $c_{i} \in \mathbb{R}$. Então:

$$
r(x)=r\left(c_{1} e_{1}+\cdots+c_{n} e_{n}\right)=\sum_{i} r\left(e_{i}\right) c_{i}^{2} \geq M \sum_{i} c_{i}^{2}>0,
$$

pois $M>0$ e $c_{i}$ não são todos nulos. Assim temos $r(x) \geq \delta>0$ para todo $x$, e podemos usar o teorema de Bonnet-Myers.

Reciprocamente suponhamos que $G$ é compacto e possui grupo fundamental finito. Como $G$ é compacto, possui uma métrica bi-invariante (veja Seção 8) tal que ad(x) é anti-adjunta, 
para todo $x$ em $\mathcal{G}$. Pelo Lema $3, r(x) \geq 0$, para todo $x$ na álgebra. Vamos mostrar que a igualdade não ocorre, para isso mostremos que $\mathcal{G}=[\mathcal{G}, \mathcal{G}]$. Lembremos que todo grupo de Lie possui um recobrimento $\pi: \tilde{G} \rightarrow G$, onde $\tilde{G}$ é um grupo de Lie simplesmente conexo e $\pi$ é um homomorfismo de grupos de Lie. Além disso, a cardinalidade do recobrimento (universal) é igual ao número de elementos do grupo fundamental. Como $G$ é compacto e o número de folhas é finito, segue que $\tilde{G}$ é compacto.

Suponhamos por absurdo que $\mathcal{G} \neq[\mathcal{G}, \mathcal{G}]$. Temos que $\mathcal{H}=[\mathcal{G}, \mathcal{G}]$ é uma subálgebra de $\mathcal{G}$ e, mais do que isso, é um ideal em $\mathcal{G}$. Pelo teorema de Lie, existe um único subgrupo conexo $H$ de $G$ tal que a álgebra de Lie de $H$ é $\mathcal{H}$. Na realidade, $H$ é subgrupo normal de $G$ de maneira que podemos fazer o quociente $G / H$. Podemos mostrar ainda que a álgebra de Lie do quociente é isomorfa à $\mathcal{G} / \mathcal{H}$ que, por sua vez, é uma álgebra de Lie abeliana e, portanto, isomorfa a um $\mathbb{R}^{n}$. Podemos considerar assim a aplicação exponencial exp : $\mathbb{R}^{n} \rightarrow G / H$ como um homomorfismo de grupos (onde estamos considerando agora o grupo aditivo $\mathbb{R}^{n}$ ). $O$ grupo $G / H$ é compacto (e completo), portanto possui uma métrica bi-invariante, segue que as exponenciais do grupo e Riemanniana coincidem. Podemos agora utilizar o teorema de Hopf-Rinow para concluir que esta aplicação é sobrejetora, e mais ainda, é um recobrimento. Com estas informações construimos o seguinte diagrama:

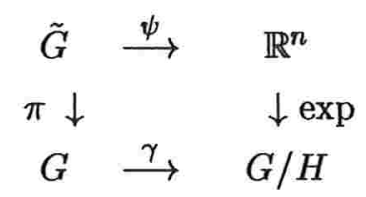

Temos então $\pi$ a aplicação de recobrimento e $\gamma: G \rightarrow G / H$ a aplicação natural que leva um elemento de $G$ em uma classe lateral em $G / H$. Pela propriedade de levantamento de recobrimentos, como $\tilde{G}$ é simplesmente conexo, temos induzido um homomorfismo de grupos de Lie $\psi$ entre $\tilde{G}$ e $\mathbb{R}^{n}$. Portanto, $\psi(\tilde{G})$ é um subgrupo compacto e conexo de $\mathbb{R}^{n}$, do que segue $\psi(\tilde{G})=\{0\}$. Mas ao mesmo tempo, $\exp (0)=e_{G / H}, \operatorname{logo} G / H=\{e\}$, absurdo.

Veremos na Seção 8 que se $G$ é compacto e simples, então a métrica bi-invariante que existe em $G$ é única a menos de multiplicação por uma constante positiva. Além disso, esta métrica possui curvatura de Ricci estritamente positiva e constante. Variedades Riemannianas que possuem curvatura de Ricci constante são usualmente chamadas de variedades de Einstein.

No caso de variedades Riemannianas em geral, mencionamos o resultado obtido por Richard Hamilton em 1982 ( [7]). Utilizando técnicas de equações diferenciais parciais, Hamilton mostrou que se $M$ é uma variedade Riemanniana simplesmente conexa, compacta de dimensão três com todas as curvaturas de Ricci estritamente positivas, então $M$ é difeomorfa à esfera $\mathbf{S}^{3}$. 
É interessante estudarmos os casos de grupos de Lie que admitem curvatura de Ricci identicamente nula ou menor ou igual a zero (constante ou não). Desta vez relacionaremos propriedades da álgebra associada com propriedades de curvatura. A próxima proposição é análoga ao Lema 3 desta seção.

Proposição 3. Se u é ortogonal à imagem $[\mathcal{G}, \mathcal{G}]$ então $r(u) \leq 0$. A igualdade vale se e somente se ad(u) é anti-adjunta.

Demonstração: Por hipótese, $u$ é ortogonal a $[\mathcal{G}, \mathcal{G}]$, logo $u^{\perp} \supseteq[\mathcal{G}, \mathcal{G}]$. Então $\mathcal{U}=u^{\perp}$ é um ideal de $\mathcal{G}$ (pois se $x \in \mathcal{G}, y \in \mathcal{U},[x, y] \in[\mathcal{G}, \mathcal{G}] \subseteq \mathcal{U}$ ) e possui codimensão 1. Queremos agora aplicar a Proposição 2 da seção anterior para $u$ e para o ideal $\mathcal{U}$. Temos que $r(u)=\sum_{i=1}^{n-1} k\left(u, v_{i}\right)$ onde $\left\{v_{1}, \cdots, v_{n-1}\right\}$ é base ortonormal para $\mathcal{U}$. Tomemos tal base consistindo de autovetores da transformação auto-adjunta $S$ (ou seja, $S v_{i}=\lambda_{i} v_{i}$ ). Seja $v$ um vetor unitário qualquer. Então:

$$
\begin{gathered}
k(u, v)=<R_{u v}(u), v>=<\nabla_{[u, v]} u-\nabla_{u} \nabla_{v} u+\nabla_{v} \nabla_{u} u, v> \\
=<\nabla_{[u, v]} u, v>-<\nabla_{u} \nabla_{v} u, v>+<\nabla_{v} \nabla_{u} u, v> \\
=<\nabla_{L(v)} u, v>-<\frac{1}{2}\left(L-L^{*}\right) \nabla_{v} u>+0 \\
=<-S L v, v>+<\frac{1}{2}\left(L-L^{*}\right) S v, v>
\end{gathered}
$$

Tomando $v$ autovetor e notando que $\left.<L v_{i}, v_{i}\right\rangle=<v_{i}, L^{*} v_{i}>=\lambda_{i}$, temos:

$$
\begin{gathered}
k\left(u, v_{i}\right)=-\left\langle S L v_{i}, v_{i}>+\frac{1}{2}<L S v_{i}, v_{i}>-\frac{1}{2}\left\langle L^{*} S v_{i}, v_{i}>\right.\right. \\
=-<S L v_{i}, v_{i}>+\frac{1}{2}\left\langle L \lambda_{i} v_{i}, v_{i}>-\frac{1}{2}\left\langle L^{*} \lambda_{i} v_{i}, v_{i}>\right.\right.
\end{gathered}
$$

e as duas últimas parcelas se cancelam. Logo $k\left(u, v_{i}\right)=-\left\langle S L v_{i}, v_{i}\right\rangle=-\left\langle\lambda_{i} L v_{i}, v_{i}\right\rangle=$ $-\lambda_{i}\left\langle L v_{i}, v_{i}\right\rangle=-\lambda_{i}^{2}$. Substituindo, temos que $r(u)=\sum_{i=1}^{n-1} k\left(u, v_{i}\right)=-\sum_{i=1}^{n-1} \lambda_{i}^{2} \leq 0$ e é igual a zero se e somente se todos os autovalores de $S$ são nulos, o que é equivalente a $S \equiv 0$, que por sua vez é equivalente a aplicação ad ser anti-adjunta.

Combinando a proposição anterior e o Lema 3 obtemos o seguinte teorema:

Teorema 2. Suponha que $\mathcal{G}$ é uma álgebra de Lie nilpotente e não comutativa. Então para qualquer métrica invariante à esquerda existe uma direção de curvatura de Ricci estritamente negativa e uma direção de curvatura de Ricci estritamente positiva. 
Demonstração: Por hipótese $\mathcal{G}$ é nilpotente. Isto significa que algum termo da série:

$$
\mathcal{G} \supset[\mathcal{G}, \mathcal{G}] \supset[\mathcal{G},[\mathcal{G}, \mathcal{G}]] \supset \cdots
$$

é zero. Escolha $u$ vetor unitário no último termo não nulo da série. Afirmamos que tal $u$ pertence ao centro de $\mathcal{G}$. De fato, seja $y \in \mathcal{G}$ qualquer. Então $[u, y] \in[$ último termo não nulo, $\mathcal{G}]=\{0\}$. Portanto, $r(u) \geq 0$ pois $a d(u)$ é anti-adjunta. Como $u \in[\mathcal{G}, \mathcal{G}], u$ não é ortogonal a $[\mathcal{G}, \mathcal{G}]$. Logo $r(u)>0$, pelo Lema 3 .

Seja $\mathcal{Z}=$ centro $\mathcal{G}$. Se $\mathcal{G}=[\mathcal{G}, \mathcal{G}]+\mathcal{Z}$, então $[\mathcal{G}, \mathcal{G}]=[\mathcal{G},[\mathcal{G}, \mathcal{G}]+\mathcal{Z}]=[\mathcal{G},[\mathcal{G}, \mathcal{G}]]$. Dessa forma, a série estabilizaria em $[\mathcal{G}, \mathcal{G}]$, absurdo. Portanto, $\mathcal{G}$ não pode ser escrito como $[\mathcal{G}, \mathcal{G}]+\mathcal{Z}$ e existe um vetor unitário $v$ tal que $v \perp[\mathcal{G}, \mathcal{G}]$ e $v$ não pertence a $\mathcal{Z}$. Afirmamos que $a d(v)$ não é anti-adjunta. Suponhamos que sim. A transformação $a d(v)$ é nilpotente, digamos, de índice $k \geq 2$ e sejam $x, y \in \mathcal{G}$ quaisquer. Então:

$$
0=<a d_{v}^{k}(x), y>=<a d_{v}\left(a d_{v}^{k-1}(x)\right), y>=-<a d_{v}^{k-1}(x), a d_{v}(y)>.
$$

Como $y$ é qualquer, podemos tomar $y=a d_{v}^{k-2}(x)$, o que resulta em contradição. Logo $a d$ não é anti-adjunta. Portanto $r(v)<0$, pela Proposição 3 .

Com maior generalidade, temos o seguinte teorema:

Teorema 3. Suponhamos que a álgebra de Lie de $G$ contém três vetores linearmente independentes $x, y, z$ tais que $[x, y]=z$. Então existe uma métrica invariante à esquerda em $G$ tal que $r(x)<0$ er $r(z)>0$.

Demonstração: Completemos $x, y, z$ a uma base de $\mathcal{G}$, denotemos tal base por $B=\left\{b_{1}=\right.$ $\left.x, b_{2}=y, b_{3}=z, \cdots, b_{n}\right\}$. Seja $\epsilon>0$ dado. Então defina:

$$
e_{1}=\epsilon b_{1}, \quad e_{2}=\epsilon b_{2}, \quad e_{i}=\epsilon^{2} b_{i}, i \geq 3 .
$$

Existe uma única métrica invariante à esquerda $\mathrm{g}_{\epsilon}$ em $G$ tal que $C=\left\{e_{1}, \cdots, e_{n}\right\}$ é ortonormal. As constantes estruturais de $\mathcal{G}_{\epsilon}$ com relação à base $C$ são dadas por:

$$
\begin{gathered}
\alpha_{12 k}=<\left[e_{1}, e_{2}\right], e_{k}>=<\left[\epsilon b_{1}, \epsilon b_{2}\right], e_{k}>=<\epsilon^{2}\left[b_{1}, b_{2}\right], e_{k}> \\
=<\epsilon^{2} b_{3}, e_{k}>=<e_{3}, e_{k}>=\delta_{3 k} .
\end{gathered}
$$

Sejam $i, j$ diferentes de 1,2 :

$$
\alpha_{i j 1}=<\left[e_{i}, e_{j}\right], e_{1}>=<\left[\epsilon^{2} b_{i}, \epsilon^{2} b_{j}\right], e_{1}>=<\epsilon^{4}\left[b_{i}, b_{j}\right], e_{1}>
$$




$$
=\epsilon^{4}<\left[b_{i}, b_{j}\right], e_{1}>=\epsilon^{4}<\sum_{i} \beta_{i j t} b_{t}, e_{1}>=\epsilon^{3} \beta_{i j 1},
$$

onde $\beta_{i j k}$ são as constantes estruturais de $\mathcal{G}$ com relação à base $B$. Analogamente, $\alpha_{i j 2}=\epsilon^{3} \beta_{i j 2}$ e para $i, j, k$ diferentes de 1,2 temos $\alpha_{i j k}=\epsilon^{2} \beta_{i j k}$.

Fazendo o limite $\epsilon \rightarrow 0$, percebe-se que as constantes estruturais (como são funções contínuas de $\epsilon$ ) convergem para um valor definido. Temos que $\alpha_{12 k} \rightarrow \delta_{3 k}$, e o restante vai a zero. Essas constantes estruturais do limite definem uma nova álgebra $\mathcal{G}_{0}$ não comutativa. Em $\mathcal{G}_{0}$, o colchete satisfaz

$$
\begin{gathered}
{\left[e_{1}, e_{2}\right]=-\left[e_{2}, e_{1}\right]=e_{3},} \\
{\left[e_{i}, e_{j}\right]=0,}
\end{gathered}
$$

caso contrário. Existe em $\mathcal{G}_{0}$ uma única métrica $\mathrm{g}_{0}$ tal que a base $C$ é ortonormal. Usando o Lema 3 e Proposição 3 , temos $r\left(e_{1}\right)<0$ pois $e_{1}$ é ortogonal a $\left[\mathcal{G}_{0}, \mathcal{G}_{0}\right]$ e $\operatorname{ad}\left(e_{1}\right)$ não é antiadjunta. E temos que $r\left(e_{3}\right)>0$ pois $a d\left(e_{3}\right)$ é anti-adjunta (é identicamente nula) e $e_{3}$ não é ortogonal a $\left[\mathcal{G}_{0}, \mathcal{G}_{0}\right]$. De fato, se $\operatorname{ad}\left(e_{1}\right)$ fossa anti-adjunta, teríamos $<\operatorname{ad}\left(e_{1}\right)\left(e_{2}\right), e_{3}>=-<$ $e_{2}, a d\left(e_{1}\right)\left(e_{3}\right)>$. Portanto:

$$
\begin{gathered}
<\left[e_{1}, e_{2}\right], e_{3}>=-<e_{2},\left[e_{1}, e_{3}\right]>\Rightarrow \\
<e_{3}, e_{3}>=0
\end{gathered}
$$

o que é absurdo. Além disso, $e_{3}$ não é ortogonal a $[\mathcal{G}, \mathcal{G}]$ pois $\left\langle e_{3},\left[e_{1}, e_{2}\right]\right\rangle=1$. Portanto, $r\left(e_{1}\right)<0<r\left(e_{3}\right)$ em $\mathcal{G}_{0}$. Como a curvatura de Ricci é um polinômio contínuo em função das constantes estruturais (pois assim o é a curvatura seccional, conforme mostramos na seção anterior), segue que $r\left(e_{1}\right)<0<r\left(e_{3}\right)$ para a métrica $\mathrm{g}_{\epsilon}$, para $\epsilon$ suficientemente próximo a zero.

Esta proposição mostra que muitos grupos de Lie possuem uma métrica invariante à esquerda cuja curvatura de Ricci admite direções de curvatura positiva e direções de curvatura negativa. Em constraste temos o exemplo 2.1 da seção anterior, em que o colchete é uma combinação linear de $x$ e $y$.

Um grupo de Lie $G$ é chamado de unimodular se sua álgebra é unimodular. Aqui definiremos uma álgebra de Lie $\mathcal{G}$ como sendo unimodular se o traço da aplicação adjunta $a d_{x}$ for identicamente nulo para todo $x$ pertencente à álgebra. Agora seja $\mathcal{G}$ uma álgebra qualquer. Vimos na. Seção 2 que vale a identidade $a d_{[x, y]}=a d_{x} a d_{y}-a d_{y} a d_{x}$, para todos $x, y$ pertencentes à álgebra. Logo $\operatorname{tr}\left(a d_{[x, y]}\right) \equiv 0$, para todos $x, y$. Segue que a aplicação $\phi: \mathcal{G} \rightarrow \mathbb{R}$ definida por $\phi(x)=\operatorname{tr}\left(a d_{x}\right)$ é um homomorfismo de álgebras de Lie. O kernel desta aplicação, 
$\mathcal{U}=\left\{x \in \mathcal{G} ; \operatorname{tr}\left(a d_{x}\right)=0\right\}$ é um ideal, isto é, $[\mathcal{G}, \mathcal{U}] \subseteq \mathcal{U}$ que contém o ideal comutador $[\mathcal{G}, \mathcal{G}]$. $\mathcal{U}$ é uma álgebra de Lie unimodular chamada de kernel unimodular de $\mathcal{G}$. O seguinte lema envolve esses conceitos com propriedades da curvatura de Ricci.

Lema 4. Seja $G$ um grupo de Lie conexo que possui uma métrica invariante à esquerda com todas as curvaturas de Ricci estritamente positivas. Então $G$ é unimodular.

Demonstração: Suponhamos por absurdo que $G$ não é unimodular. Podemos escolher um vetor unitário $b$ ortogonal a kernel unimodular de $\mathcal{G}$ (de fato, se não houvesse tal vetor, teríamos que o kernel unimodular seria o próprio grupo, absurdo pois estamos supondo $G$ não unimodular). Então $\operatorname{tr}\left(a d_{b}\right) \neq 0$ e se $a d_{b}$ fosse anti-adjunta teríamos $a d_{b}=-a d_{b}^{*}$ e portanto. $\operatorname{tr}\left(a d_{b}\right)=-\operatorname{tr}\left(a d_{b}^{*}\right)=-\operatorname{tr}\left(a d_{b}\right)$, absurdo. Logo $a d_{b}$ não é anti-adjunta. Pela Proposição 3 , $r(b)<0$, absurdo. 


\section{Curvatura Escalar}

Seja $\left\{e_{1}, \cdots, e_{n}\right\}$ uma base de vetores tangentes a $G$ no ponto $p$. O número real:

$$
\rho=r\left(e_{1}\right)+\cdots+r\left(e_{n}\right)=2 \sum_{i<j} k\left(e_{i}, e_{j}\right)
$$

é chamado de curvatura escalar no ponto $p$.

No próximo lema estamos considerando a curvatura escalar em $\mathcal{G}$ e denotando-a por $\rho=\rho(\mathcal{G})$. Com a notação da Proposição 2 da Seção $1, \mathcal{U}$ é uma álgebra de Lie com a métrica induzida por $\mathcal{G}$ e podemos considerar $\rho(\mathcal{U})$. Neste caso temos o seguinte lema:

Lema 5. A curvatura escalar $\rho(\mathcal{G})$ associada à álgebra de Lie $\mathcal{G}$ é igual a $\rho(\mathcal{U})-\operatorname{tr}\left(S^{2}\right)-(\operatorname{tr}(S))^{2}$.

Demonstração: Sejam $u, v$ vetores ortonormais em $\mathcal{U}$. Comparando a curvatura seccional $k(u, v)$ em $\mathcal{G}$ e $\bar{k}(u, v)$ em $\mathcal{U}$, temos que $k(u, v)=\bar{k}(u, v)+\langle S u, v\rangle^{2}-\langle S u, u\rangle\langle S v, v\rangle$ (é a equação de Gauss). Escolhamos agora uma base ortonormal $\left\{u_{1}, \cdots, u_{n-1}\right\}$ de $\mathcal{U}$ formada de autovetores de $S$. Segue que $k\left(u_{i}, u_{j}\right)=\bar{k}\left(u_{i}, u_{j}\right)-\lambda_{i} \lambda_{j}$, para $i \neq j$. Fixando $i$ e somando em $j$, temos que:

$$
\begin{gathered}
\sum_{j} k\left(u_{i}, u_{j}\right)=\sum_{j} \bar{k}\left(u_{i}, u_{j}\right)-\sum_{j} \lambda_{i} \lambda_{j} \Rightarrow \\
r\left(u_{i}\right)=\bar{r}\left(u_{i}\right)-\lambda_{i} \operatorname{tr}(S) .
\end{gathered}
$$

Temos que $\left\{b, u_{1}, \cdots, u_{n-1}\right\}$ é uma base ortonormal de $\mathcal{G}$. Vimos na Proposição 3 da Seção 2 que $r(b)=-\operatorname{tr}\left(S^{2}\right)$. Logo:

$$
r(b)+r\left(u_{1}\right)+\cdots+r\left(u_{n-1}\right)=-\operatorname{tr}\left(S^{2}\right)+\bar{r}\left(u_{1}\right)-\lambda_{1} \operatorname{tr}(S)+\cdots+\bar{r}\left(u_{n-1}\right)-\lambda_{n-1} \operatorname{tr}(S)
$$

portanto,

$$
\rho(\mathcal{G})=\rho(\mathcal{U})-\operatorname{tr}(S)^{2}-\operatorname{tr}\left(S^{2}\right)
$$

Teorema 4. Seja $G$ um grupo de Lie solúvel. Então toda métrica invariante à esquerda em $G$ ou é flat, ou possui curvatura escalar estritamente negativa.

Demonstração: A demonstração será feita por indução sobre a dimensão da álgebra $\mathcal{G}$. Seja $n$ tal dimensão. O caso $n=1$ é imediato pois assim a álgebra é abeliana e, portanto, qualquer métrica invariante à esquerda é flat. Estamos supondo $\mathcal{G} \neq\{0\}$ solúvel. Então temos que $\mathcal{G} \neq[\mathcal{G}, \mathcal{G}]$ pois se fosse igual a série derivada estabilizaria, absurdo. Temos que $[\mathcal{G}, \mathcal{G}]$ é um ideal em $\mathcal{G}$ e existe um subespaço $\mathcal{U}$ de $\mathcal{G}$ de codimensão 1 contendo $[\mathcal{G}, \mathcal{G}]$. Na realidade $\mathcal{U}$ é um ideal em $\mathcal{G}$ e, por isso, é uma subálgebra solúvel. Pela hipótese de indução, temos que $\rho(\mathcal{U}) \leq 0$. 
Portanto, $\rho(\mathcal{G}) \leq-\operatorname{tr}\left(S^{2}\right)-\operatorname{tr}(S)^{2} \leq 0$. Ou seja, $\rho(\mathcal{G})<0$ ou $\rho(\mathcal{G})=0$. Queremos verificar que se $\rho(\mathcal{G})=0$, então a métrica é flat. A igualdade vale se $\rho(\mathcal{U})=0$ e se $-\operatorname{tr}\left(S^{2}\right)-\operatorname{tr}(S)^{2}=0$. Como a matriz de $S$ é diagonal, tal igualdade implica que $S=0$. Vamos mostrar que se tais fatos ocorrem, então $\mathcal{G}$ é flat, isto é, o tensor de curvatura $R$ é identicamente nulo.

Se $S=0$, pela Proposição 2 da seção 1 (e usando toda aquela notação), temos que $\nabla_{u} v=\bar{\nabla}_{u} v$ para cada $u, v$ no ideal $\mathcal{U}$. Portanto, $R_{u v}(w)=\bar{R}_{u v}(w)$, para $u, v$ e $w$ no ideal. Como $\rho(\mathcal{U})$ é nulo, temos pela hipótese de indução que $\mathcal{U}$ é flat e então $\bar{R}=0$, o que implica $R_{u v}(w)=0$ para todos $u, v, w$ em $\mathcal{U}$. Também pela proposição citada, temos $\nabla_{u} b=-S u$, logo se $S=0$, segue que $\nabla_{u} b=0$, para todo $u \in \mathcal{U}$. Se $x$ pertence a $\mathcal{G}$, então $x=u+k b$ onde $u \in \mathcal{U}$ e $k$ é uma constante e $b$ é um vetor unitário ortogonal a $\mathcal{U}$. Logo $\nabla_{x} b=\nabla_{u+k b} b=\nabla_{u} b+k \nabla_{b} b=0$. Segue que $R_{x y}(b)=0$ para quaisquer $x, y$ em $\mathcal{G}$. Sabemos que vale $\left\langle R_{x y}(b), z\right\rangle=\left\langle R_{b z}(x), y\right\rangle$. Portanto $R_{b z}=0$ para qualquer $z$. Podemos calcular $R_{x y}(z)$ para quaisquer $x, y, z$ em $\mathcal{G}$; basta escrever cada elemento como $u+k b$ com $u \in \mathcal{U}$ e $k$ uma constante, usar que $R$ é linear em $x, y$ e $z$ e usar o que mostramos anteriormente. Segue que $R$ é identicamente nulo. Portanto $\mathcal{G}$ é flat e isto termina a demonstração.

R. Azencott e E. Wilson mostraram em [2] que se um grupo de Lie $G$ possui uma métrica invariante à esquerda com todas as curvaturas seccionais menores ou iguais a zero, então ele é solúvel. Se além disso o grupo é unimodular então tal métrica com todas as curvaturas seccionais menores ou iguais a zero é, na realidade, flat. Ou seja, nesse caso toda métrica invariante à esquerda em $G$ possui algum plano de curvatura seccional estritamente positivo, a não ser que tal métrica seja de curvatura identicamente nula. Suponhamos que $G$ é unimodular e solúvel. Pelo resultado anterior, toda métrica invariante à esquerda em $G$ ou é flat ou possui curvatura escalar estritamente negativa. Se não é flat, como $G$ é unimodular, pelo resultado de Azencott e Wilson, existem planos de curvatura seccional esritamente positiva. Ao mesmo tempo se a métrica não é flat e como $G$ é solúvel, pelo resultado demonstrado anteriormente existem planos de curvarura seccional estritamente negativa. Mostramos assim o seguinte corolário:

Corolário 2. Seja $G$ um grupo de Lie solúvel e unimodular. Então toda métrica invariante à esquerda em $G$ ou é flat ou possui planos com curvatura seccional positiva e planos com curvatura seccional negativa.

Um critério muito geral está contido no próximo teorema:

Teorema 5. Se a álgebra de Lie $\mathcal{G}$ de $G$ é não comutativa, então $G$ possui uma métrica invariante à esquerda com curvatura escalar estritamente negativa. 
Demonstração: Observe que se $x, y, \mathrm{e}[x, y]$ fossem sempre linearmente dependentes então estaríamos lidando com o exemplo da seção 1 e neste caso mostramos que a curvatura seccional é estritamente negativa, para qualquer escolha de métrica invariante à esquerda. Podemos excluir esse caso e supor que existem vetores linearmente independentes $x, y$ e $z$ na álgebra de Lie de $G$ tais que $z=[x, y]$. Completemos $x, y, z$ a uma base de $\mathcal{G}$, denotemos tal base por $B=\left\{b_{1}=x, b_{2}=y, b_{3}=z, \cdots, b_{n}\right\}$. Seja $\epsilon>0$ dado. Então defina:

$$
e_{1}=\epsilon b_{1}, e_{2}=\epsilon b_{2}, e_{i}=\epsilon^{2} b_{i}, i \geq 3
$$

Existe uma única métrica invariante à esquerda $g_{\epsilon}$ em $G$ tal que $C=\left\{e_{1}, \cdots, e_{n}\right\}$ é ortonormal.

Analogamente a que foi feito no Teorema 3 da seção anterior, podemos estudar as constantes estruturais $\alpha_{i j k}$ de $\mathcal{G}$ com relação à $C$. Sabemos assim que $\alpha_{12 k}=\delta_{3 k}$. e para $i, j \neq 1,2$, $\alpha_{i j 1}=\epsilon^{3} \beta_{i j 1}$, onde $\beta_{i j 1}$ são as constantes estruturais de $\mathcal{G}$ com relação à base $B$. Temos também $\alpha_{i j 2}=\epsilon^{3} \beta_{i j 2}$. Para $i, j, k \neq 1,2$ temos $\alpha_{i j k}=\epsilon^{2} \beta_{i j k}$. Façamos agora o limite $\epsilon \rightarrow 0$. As constantes estruturais de $\mathcal{G}$ tendem para valores definidos de maneira que temos definida uma nova álgebra $\mathcal{G}_{0}$, equipada com uma métrica $\mathrm{g}_{0}$ tal que $C$ é ortonormal. Observe agora que no limite, $\alpha_{123}=<\left[e_{1}, e_{2}\right], e_{3}>=1$, logo a álgebra $\mathcal{G}_{0}$ não é comutativa. Observe também que no limite temos que $\alpha_{i j k}=0$ para os outros casos. Portanto $\left[e_{i},\left[e_{j}, e_{k}\right]\right]=0$ para todo $i, j, k$, o que mostra que $\mathcal{G}_{0}$ é uma álgebra nilpotente e, portanto, solúvel. Pelo resultado anterior, toda métrica invariante à esquerda em $G_{0}$ ou é flat ou possui curvatura escalar estritamente negativa. Como a álgebra de Lie $\mathcal{G}_{0}$ de $G_{0}$ não é comutativa, pelo Teorema 3 da Seção 3, toda métrica invariante à esquerda em $G_{0}$ possui uma direção de curvatura de Ricci estritamente positiva e uma direção de curvatura de Ricci estritamente negativa. Logo $G_{0}$ não é flat, e assim $\rho\left(\mathcal{G}_{0}\right)<0$. Lembremos agora pelo Lema 1 da Seção 1 que a curvatura seccional é um polinômio contínuo em função das constantes estruturais da álgebra, portanto assim o é a curvatura escalar. Então $\rho\left(\mathcal{G}, \mathbf{g}_{\epsilon}\right)<0$ para $\epsilon$ suficientemente pequeno.

Na. Seção 10 estudaremos o caso de curvatura escalar estritamente positiva. Veremos que se $G$ contém um subgrupo compacto não comutativo então $G$ admite uma métrica invariante à esquerda de curvatura escalar estritamente positiva. Este resultado foi apresentado por N. Wallach. 


\section{Grupos de Lie de Dimensão Três}

Seja $\mathcal{G}$ um espaço vetorial de dimensão três. Uma orientação para $\mathcal{G}$ é uma classe de equivalência de bases ordenadas, onde a base ordenada $\left\{b_{1}, b_{2}, b_{3}\right\}$ tem a mesma orientação da base $\left\{b_{1}^{\prime}, b_{2}^{\prime}, b_{3}^{\prime}\right\}$ se $b_{i}^{\prime}=\sum_{i j} a_{i j} b_{j} \operatorname{com} \operatorname{det}\left(a_{i j}\right)>0$ e tem orientação oposta $\operatorname{se} \operatorname{det}\left(a_{i j}\right)<0$. Seja então $G$ um grupo de Lie conexo de dimensão 3 com uma métrica invariante à esquerda e seja $\mathcal{G}$ sua álgebra com uma orientação escolhida, de maneira que podemos definir o produto vetorial em $\mathcal{G}$. Para $u$ e $v$ em $\mathcal{G}$, o produto vetorial de $u$ por $v$, denotado por $u \times v$, é tal que $u \times v$ é ortogonal a $u$ e $v$ e possui comprimento igual à raiz quadrada de $\langle u, u\rangle\langle v, v\rangle-\langle u, v\rangle^{2}$. A direção de $u \times v$ fica determinada quando assumimos que a tripla $\{u, v, u \times v\}$ é orientada positivamente sempre que $u$ e $v$ são linearmente independentes. Para $G$ e $\mathcal{G}$ dessa maneira, temos o seguinte lema:

Lema 6. $O$ colchete em $\mathcal{G}$ está relacionado com o produto vetorial através de $[u, v]=L(u \times v)$, onde $L$ é uma aplicação linear de $\mathcal{G}$ em $\mathcal{G}$ unicamente definida $e[\cdot, \cdot]: \mathcal{G} \times \mathcal{G} \rightarrow \mathcal{G}$ é a operação colchete da álgebra. Além disso, $G$ é unimodular se e somente se $L$ é auto-adjunta.

Demonstração: Seja $\left\{e_{1}, e_{2}, e_{3}\right\}$ uma base ortonormal de $\mathcal{G}$ orientada positivamente. Podemos definir uma única transformação linear $L: \mathcal{G} \rightarrow \mathcal{G}$ através dos seus valores em tal base:

$$
\begin{aligned}
& L\left(e_{1}\right)=\left[e_{2}, e_{3}\right], \\
& L\left(e_{2}\right)=\left[e_{3}, e_{1}\right], \\
& L\left(e_{3}\right)=\left[e_{1}, e_{2}\right] .
\end{aligned}
$$

Temos que $L\left(e_{1} \times e_{2}\right)=L\left(e_{3}\right)=\left[e_{1}, e_{2}\right]$, e o mesmo vale para os outros elementos da base. Portanto, pela linearidade, $L(u \times v)=[u, v]$ para todos $u, v$ em $\mathcal{G}$. Podemos escrever $L\left(e_{i}\right)=$ $\sum_{j} a_{i j} e_{j}$. Por exemplo, temos $L\left(e_{1}\right)=a_{11} e_{1}+a_{12} e_{2}+a_{13} e_{3}$. Além disso, temos:

$$
\begin{gathered}
a d\left(e_{1}\right)\left(e_{1}\right)=0 e_{1}+0 e_{2}+0 e_{3}, \\
\operatorname{ad}\left(e_{1}\right)\left(e_{2}\right)=\left[e_{1}, e_{2}\right]=L\left(e_{3}\right)=a_{31} e_{1}+a_{32} e_{2}+a_{33} e_{3}, \\
\operatorname{ad}\left(e_{1}\right)\left(e_{3}\right)=\left[e_{1}, e_{3}\right]=-L\left(e_{2}\right)=-a_{21} e_{1}-a_{22} e_{2}-a_{23} e_{3},
\end{gathered}
$$

portanto $\operatorname{tr} \operatorname{ad}\left(e_{1}\right)=-a_{23}+a_{32}$. Analogamente temos que $\operatorname{tr}$ ad $\left(e_{2}\right)=-a_{31}+a_{13}$ e $\operatorname{tr}$ ad $\left(e_{3}\right)=$ $-a_{12}+a_{21}$. O grupo $G$ é unimodular se e somente se $t r a d \equiv 0$. Logo, se e somente se $a_{23}=a_{32}$, $a_{31}=a_{13}$ e $a_{12}=a_{21}$. Isto implica que a matriz $a_{i j}$ é simétrica o que é equivalente a dizer que $L$ é auto-adjunta. 
Mostramos que se $G$ é unimodular, temos $L$ auto-adjunta. Portanto, existe uma base ortonormal (que podemos assumir ser orientada positivamente) $\left\{e_{1}, e_{2}, e_{3}\right\}$ de $\mathcal{G}$ formada de auto-vetores de $L$. Neste caso temos, por exemplo, $\left[e_{1}, e_{2}\right]=L\left(e_{1} \times e_{2}\right)=L\left(e_{3}\right)=\lambda_{3} e_{3}$. Analogamente, obte$\operatorname{mos}\left[e_{3}, e_{1}\right]=\lambda_{2} e_{2}$ e $\left[e_{2}, e_{3}\right]=\lambda_{1} e_{1}$. Conseguimos obter o comportamento do colchete em um grupo de Lie unimodular $G$ de dimensão 3 . Observe que se mudarmos a orientação da álgebra, então o produto vetorial mudará de sinal e consequentemente $L$ e seus autovalores mudarão de sinal. Gostaríamos de saber como se comporta a curvatura neste caso. Para isto introduzimos a. seguinte notação:

$$
\mu_{i}=\frac{1}{2}\left(\lambda_{1}+\lambda_{2}+\lambda_{3}\right)-\lambda_{i}
$$

Temos o seguinte lema:

Lema 7. $A$ base $\left\{e_{1}, e_{2}, e_{3}\right\}$ escolhida como anteriormente diagonaliza a forma quadrática de Ricci. As curvaturas principais de Ricci são dadas por $r\left(e_{1}\right)=2 \mu_{2} \mu_{3}, r\left(e_{2}\right)=2 \mu_{1} \mu_{3}$ er $r\left(e_{3}\right)=$ $2 \mu_{1} \mu_{2}$.

Demonstração: Primeiro, mostremos que vale a seguinte fórmula:

$$
\nabla_{e_{i}} v=\mu_{i} e_{i} \times v
$$

para todo $v \in \mathcal{G}$. Pela linearidade do operador $\nabla$ e do produto vetorial, basta verificar a fórmula para os elementos da base $\left\{e_{1}, e_{2}, e_{3}\right\}$. Usando que $\nabla_{e_{i}} e_{j}=\sum_{k} \frac{1}{2}\left(\alpha_{i j k}-\alpha_{j k i}+\alpha_{k i j}\right) e_{k}$, temos:

$$
\begin{gathered}
\nabla_{e_{1}} e_{2}=\sum_{k} \frac{1}{2}\left(\alpha_{12 k}-\alpha_{2 k 1}+\alpha_{k 12}\right) e_{k} \\
=\frac{1}{2}\left[\left(\alpha_{121}-\alpha_{211}+\alpha_{112}\right) e_{1}+\left(\alpha_{122}-\alpha_{221}+\alpha_{212}\right) e_{2}+\left(\alpha_{123}-\alpha_{231}+\alpha_{312}\right) e_{3}\right] \\
=\frac{1}{2}\left(\alpha_{123}-\alpha_{231}+\alpha_{312}\right) e_{3},
\end{gathered}
$$

pois as duas primeiras somas são zero já que $\alpha_{121}=<\left[e_{1}, e_{2}\right], e_{1}>=<\lambda_{3} e_{3}, e_{1}>=0$ e $\alpha_{122}=<$ $\left[e_{1}, e_{2}\right], e_{2}>=0$. Observemos que $\alpha_{123}=<\left[e_{1}, e_{2}\right], e_{3}>=\lambda_{3}, \alpha_{231}=\lambda_{1}$ e $\alpha_{312}=\lambda_{2}$. Logo:

$$
\nabla_{e_{1}} e_{2}=\frac{1}{2}\left(\lambda_{3}-\lambda_{1}+\lambda_{2}\right) e_{3}=\mu_{1} e_{3}=\mu_{1}\left(e_{1} \times e_{2}\right) \text {. }
$$

Analogamente mostramos que vale para outras escolhas de elementos da base $\left\{e_{1}, e_{2}, e_{3}\right\}$. É fácil verificar também para os elementos da base a seguinte identidade:

$$
e_{1} \times\left(e_{2} \times v\right)-e_{2} \times\left(e_{1} \times v\right)-\left(e_{1} \times e_{2}\right) \times v=0 .
$$


Substituindo a fórmula que encontramos para a conexão na fórmula do tensor de Riemann:

$$
\begin{gathered}
R\left(e_{1}, e_{2}\right) v=\nabla_{\lambda_{3} e_{3}} v-\nabla_{e_{1}}\left(\mu_{2} e_{2} \times v\right)+\nabla_{e_{2}}\left(\mu_{1} e_{1} \times v\right) \\
=\lambda_{3} \mu_{3} e_{3} \times v-\mu_{2} \mu_{1} e_{1} \times\left(e_{2} \times v\right)+\mu_{1} \mu_{2} e_{2} \times\left(e_{1} \times v\right) \\
=\left(\lambda_{3} \mu_{3}-\mu_{2} \mu_{1}\right) e_{3} \times v
\end{gathered}
$$

Analogamente mostramos que:

$$
\begin{aligned}
& R\left(e_{1}, e_{3}\right) v=\left(\mu_{1} \mu_{3}-\lambda_{2} \mu_{2}\right) e_{2} \times v \\
& R\left(e_{2}, e_{3}\right) v=\left(\lambda_{1} \mu_{1}-\mu_{3} \mu_{2}\right) e_{1} \times v
\end{aligned}
$$

Assim conseguimos encontrar a transformação auto-adjunta de Ricci $\hat{r}, \hat{r}\left(e_{1}\right), \hat{r}\left(e_{2}\right)$ e $\hat{r}\left(e_{3}\right)$. Por exemplo, temos:

$$
\begin{gathered}
\hat{r}\left(e_{1}\right)=\left(\lambda_{3} \mu_{3}-\mu_{1} \mu_{2}\right) e_{1}+\left(\lambda_{2} \mu_{2}-\mu_{1} \mu_{3}\right) e_{1} \\
=\left(\mu_{3}\left(\lambda_{3}-\mu_{1}\right)+\mu_{2}\left(-\mu_{1}+\lambda_{2}\right)\right) e_{1}=\left(\mu_{2} \mu_{3}+\mu_{3} \mu_{2}\right) e_{1},
\end{gathered}
$$

lembrando que $\lambda_{3}-\mu_{1}=\mu_{2}$ e $\lambda_{2}-\mu_{1}=\mu_{3}$. Analogamente calculamos $\hat{r}\left(e_{2}\right)=\left(2 \mu_{1} \mu_{3}\right) e_{2}$ e $\hat{r}\left(e_{3}\right)=\left(2 \mu_{1} \mu_{2}\right) e_{3}$. Portanto, $\left\{e_{1}, e_{2}, e_{3}\right\}$ é uma base de $\mathcal{G}$ formada de autovetores de $\hat{r}$; segue que $r\left(e_{1}\right)=2 \mu_{2} \mu_{3}, r\left(e_{2}\right)=2 \mu_{1} \mu_{3}$ e $r\left(e_{3}\right)=2 \mu_{1} \mu_{2}$.

Em particular, a curvatura escalar é dada por $\rho=2\left(\mu_{2} \mu_{3}+\mu_{1} \mu_{3}+\mu_{1} \mu_{2}\right)$. O produto $r\left(e_{1}\right) r\left(e_{2}\right) r\left(e_{3}\right)=8\left(\mu_{1} \mu_{2} \mu_{3}\right)^{2}$ é sempre maior ou igual a zero e será igual a zero se algum $r\left(e_{i}\right)$ for nulo. Suponhamos sem perda de generalidade que $r\left(e_{1}\right)=0$. Então nesse caso, ou $\mu_{2}$ ou $\mu_{3}$ são iguais a zero. Suponhamos (s.p.g.) que $\mu_{2}=0$. então temos $r\left(e_{3}\right)=2 \mu_{1} \mu_{2}=0$. Logo, se o determinante for zero, então pelo menos duas curvaturas principais de Ricci são zero.

Agora suponhamos que o produto $r\left(e_{1}\right) r\left(e_{2}\right) r\left(e_{3}\right)$ é diferente de zero. Isto quer dizer que todos os $r\left(e_{i}\right)$ são não-nulos. Por exemplo, $r\left(e_{1}\right) \neq 0$. Então $2 \mu_{2} \mu_{3} \neq 0$. Logo $\mu_{2} \neq 0$ e $\mu_{3} \neq 0$. Podemos fazer todas as inversas, já que são todos não nulos. De fato, temos:

$$
\mu_{2}=\frac{1}{2} r\left(e_{1}\right) \mu_{3}^{-1}=\frac{1}{2} r\left(e_{1}\right) 2 \mu_{1} r\left(e_{2}\right)^{-1}=r\left(e_{1}\right) r\left(e_{2}\right)^{-1} \mu_{1}=r\left(e_{1}\right) r\left(e_{2}\right)^{-1} \frac{1}{2} r\left(e_{3}\right) \mu_{2}^{-1} .
$$


Portanto, $\left(\mu_{2}\right)^{2}=\frac{1}{2} r\left(e_{1}\right) r\left(e_{2}\right)^{-1} r\left(e_{3}\right)$. Analogamente, $\left(\mu_{3}\right)^{2}=\frac{1}{2} r\left(e_{1}\right) r\left(e_{3}\right)^{-1} r\left(e_{2}\right)$ e $\left(\mu_{1}\right)^{2}=$ $\frac{1}{2} r\left(e_{2}\right) r\left(e_{1}\right)^{-1} r\left(e_{3}\right)$. Lembre-se também que $\mu_{1}+\mu_{2}=\lambda_{3}, \mu_{2}+\mu_{3}=\lambda_{1}$ e $\mu_{1}+\mu_{3}=\lambda_{2}$. Então podemos escrever cada $\lambda_{i}$ em função das curvaturas principais de Ricci $r\left(e_{i}\right)$.

Se mudamos a orientação de $\mathcal{G}$, então o produto vetorial muda de sinal, portanto $L$ muda de sinal e assim cada $\lambda_{i}$ muda de sinal, portanto cada $\mu_{i}$ muda de sinal. Cada $r\left(e_{i}\right)$ é produto de dois $\mu_{i}$, logo o sinal de $r\left(e_{i}\right)$ é mantido, como se espera.

Sejam $\eta, \zeta, \xi$ constantes quaisquer. Suponhamos agora que escolhemos uma métrica em $G$ tal que a base $\left\{\eta \zeta e_{1}, \xi \zeta e_{2}, \xi \eta e_{3}\right\}$ é ortonormal. Temos a aplicação $L: \mathcal{G} \rightarrow \mathcal{G}$ definida por:

$$
\begin{gathered}
L\left(\eta \zeta e_{1}\right)=\left[\xi \zeta e_{2}, \xi \eta e_{3}\right]=\left(\xi^{2} \lambda_{1}\right) \zeta \eta e_{1}, \\
L\left(\xi \zeta e_{2}\right)=\left[\xi \eta e_{3} \eta \zeta e_{1}\right]=\left(\eta^{2} \lambda_{2}\right) \xi \zeta e_{2}, \\
L\left(\xi \eta e_{3}\right)=\left(\zeta^{2} \lambda_{3}\right) \xi \eta e_{3} .
\end{gathered}
$$

Portanto as constantes estruturais da nova base $\left\{\eta \zeta e_{1}, \xi \zeta e_{2}, \xi \eta e_{3}\right\}$ são múltiplos positivos de $\lambda_{i}$, ou seja, podemos alterar um pouco a métrica de maneira que os autovalores de $L$ se tornem $+1,-1$, ou 0 . Além disso, podemos assumir que no máximo uma das constantes $\lambda_{1}, \lambda_{2}, \lambda_{3}$ é negativa já que caso duas delas forem negativas, mudamos a orientação da álgebra de maneira que todas elas mudam de sinal. Contabilizando, temos seis possibilidades para os sinais de $\lambda_{1}$, $\lambda_{2}$ e $\lambda_{3}$. Exibiremos agora exemplos de cada uma delas. Nos próximos exemplos, denotaremos por $M(n, \mathbf{K})$ o conjunto das matrizes $n \times n$ com coeficientes em um corpo $\mathbf{K}$ e por $G l(n, \mathbf{K})$ o conjunto das matrizes $n \times n$ de determinante não nulo com coeficientes em $\mathbf{K}$.

\section{$\mathbf{5 . 1} S l(2, \mathbb{R})$}

O conjunto $S l(2, \mathbb{R})$ é formado pelas matrizes $2 \times 2$ reais com determinante igual a 1 ; não é difícil ver que tal conjunto é um subgrupo abstrato de $G l(2, \mathbb{R})$. De fato, sejam $A, B$ pertencentes a. $S l(2, \mathbb{R})$, então $\operatorname{det}(A B)=\operatorname{det} A \operatorname{det} B=1, \operatorname{edet}\left(A^{-1}\right)=(\operatorname{det} A)^{-1}=1$. Portanto $A B, A^{-1} \in$ $S l(2, \mathbb{R})$. Além disso, podemos mostrar que $S l(2, \mathbb{R})$ é uma subvariedade fechada mergulhada de $G l(2, \mathbb{R})$.

Consideremos a função $f: G l(2, \mathbb{R}) \rightarrow \mathbb{R}$ dada pelo determinante $f(X)=\operatorname{det} X$. Temos que $S l(2, \mathbb{R})=\{X \in G l(2, \mathbb{R}) ; f(X)-1=0\}$. Se mostrarmos que tal função é independente em $S l(2, \mathbb{R})$ então $S l(2, \mathbb{R})$ é subvariedade fechada mergulhada de $G l(2, \mathbb{R})$. Mostrar que tal função é independente em $S l(2, \mathbb{R})$ significa mostrar que a diferencial $(d f)_{x}$ é linearmente independente 
(não-nula) para todo $x \in S l(2, \mathbb{R})$. De fato, temos:

$$
d f=\left|\begin{array}{ll}
d x_{1}^{1} & x_{2}^{1} \\
d x_{1}^{2} & x_{2}^{2}
\end{array}\right|+\left|\begin{array}{ll}
x_{1}^{1} & d x_{2}^{1} \\
x_{1}^{2} & d x_{2}^{2}
\end{array}\right|
$$

e portanto:

$$
(d f)_{x}=\left|\begin{array}{ll}
\left(d x_{1}^{1}\right)_{x} & x_{2}^{1}(x) \\
\left(d x_{1}^{2}\right)_{x} & x_{2}^{2}(x)
\end{array}\right|+\left|\begin{array}{ll}
x_{1}^{1}(x) & \left(d x_{2}^{1}\right)_{x} \\
x_{1}^{2}(x) & \left(d x_{2}^{2}\right)_{x}
\end{array}\right|
$$

onde se $a$ é uma matriz qualquer em $G l(2, \mathbb{R})$, então $x_{i}^{j}(a)=a_{i}^{j}$. Se existisse um elemento $x \in S l(2, \mathbb{R})$ tal que $(d f)_{x} \equiv 0$, então poderíamos ter $(d f)_{x}(x)=2 \operatorname{det} x=0$, absurdo. Logo $(d f)_{x}$ é linearmente independente e $S l(2, \mathbb{R})$ é subvariedade fechada mergulhada de $G l(2, \mathbb{R})$. Logo, as operações de grupo de Lie de $G l(2, \mathbb{R})$, a saber, $G l(2, \mathbb{R}) \times G l(2, \mathbb{R}) \rightarrow G l(2, \mathbb{R})$ que faz $(A, B) \mapsto A \cdot B$ e $G l(2, \mathbb{R}) \rightarrow G l(2, \mathbb{R})$ tal que $A \mapsto A^{-1}$, quando restritas à $S l(2, \mathbb{R})$, continuam $C^{\infty}$. Logo $S l(2, \mathbb{R})$ é subgrupo de Lie fechado de $G l(2, \mathbb{R})$ de dimensão 3 .

Seja $X \in M(2, \mathbb{R})$, onde lembramos que estamos considerando $M(2, \mathbb{R})$ como álgebra de Lie de $G l(2, \mathbb{R})$, e $\exp t X$ subgrupo a um parâmetro de $G l(2, \mathbb{R})$. Lembremos que se $\phi: G \rightarrow H$ é um homomorfismo de grupos de Lie então vale que $\phi(\exp t X)=\exp t\left((d \phi)_{e}(X)\right)$, onde $X$ pertence à álgebra de Lie de $G$. No caso deste exemplo temos que $\operatorname{det}(\exp t X)=\exp t(\operatorname{tr} X)$, onde $X \in M(2, \mathbb{R})$. Lembremos agora que um elemento $X \in M(2, \mathbb{R})$ pertence à álgebra de Lie de $S l(2, \mathbb{R})$ se e somente se $\exp t X$ pertence a $S l(2, \mathbb{R})$ para todo $t$ real. Mas isso ocorre se e somente se $\operatorname{tr} X=0$. Portanto a álgebra de Lie de $S l(2, \mathbb{R})$, que denotaremos por $\mathcal{S L}(2, \mathbb{R})$ é o conjunto:

$$
\mathcal{S} \mathcal{L}(2, \mathbb{R})=\{X \in M(2, \mathbb{R}) ; \operatorname{tr} X=0\}
$$

que é uma subálgebra da álgebra de Lie de $G l(2, \mathbb{R})$ de dimensão 3 . Não é difícil ver que o conjunto

$$
\left\{e_{1}=\frac{1}{2}\left(\begin{array}{cc}
1 & 0 \\
0 & -1
\end{array}\right), e_{2}=\frac{1}{2}\left(\begin{array}{ll}
0 & 1 \\
0 & 0
\end{array}\right), e_{3}=\frac{1}{2}\left(\begin{array}{ll}
0 & 0 \\
1 & 0
\end{array}\right)\right\}
$$

é uma base de $\mathcal{S} \mathcal{L}(2, \mathbb{R})$, assim como $\left\{e_{1}, e_{2}-e_{3}, e_{2}+e_{3}\right\}$; chamemos tal base de $\left\{b_{1}, b_{2}, b_{3}\right\}$. É imediato verificar que:

$$
\begin{gathered}
{\left[b_{1}, b_{2}\right]=\left[e_{1}, e_{2}-e_{3}\right]=\left[e_{1}, e_{2}\right]-\left[e_{1}, e_{3}\right]=e_{2}+e_{3}=b_{3},} \\
{\left[b_{3}, b_{1}\right]=\left[e_{2}+e_{3}, e_{1}\right]=\left[e_{2}, e_{1}\right]+\left[e_{3}, e_{1}\right]=-e_{2}+e_{3}=-b_{2},} \\
{\left[b_{2}, b_{3}\right]=\left[e_{2}-e_{3}, e_{2}+e_{3}\right]=2\left[e_{2}, e_{3}\right]=2 e_{1}=2 b_{1} .}
\end{gathered}
$$

O grupo $S l(2, \mathbb{R})$ é unimodular pois $\mathcal{S L}(2, \mathbb{R})$ é uma álgebra unimodular, ou seja, o traço da aplicação adjunta $a d_{x}$ é identicamente nulo, para cada $x \in \mathcal{S L}(2 \mathbb{R})$. Temos que $a d_{x}(y)=[x, y]$, 
e seja $x=\left(\begin{array}{ll}a_{11} & a_{12} \\ a_{21} & a_{22}\end{array}\right)$ um elemento qualquer da álgebra. Calculando $a_{d}\left(e_{i}\right)$ para $i=1,2,3$, não é difícil encontrar a matriz da transformação adjunta, que é dada por:

$$
\left[a d_{x}\right]=\left(\begin{array}{ccc}
0 & -a_{21} & a_{12} \\
-2 a_{12} & \left(a_{11}-a_{22}\right) & 0 \\
2 a_{21} & 0 & \left(a_{22}-a_{11}\right)
\end{array}\right)
$$

que possui traço nulo, para qualquer $x$ pertencente à álgebra. Portanto $\mathcal{S L}(2, \mathbb{R})$ é unimodular.

Vamos mostrar que o grupo $S l(2, \mathbb{R})$ é conexo. Seja $\mathbb{R}^{+}$o conjunto dos número reais estritamente positivos. Vamos definir uma aplicação $\phi: \mathbb{R}^{+} \times S l(2, \mathbb{R}) \rightarrow G l^{+}(2, \mathbb{R})$ como sendo $\phi(\lambda, A)=\lambda \cdot A$. Essa aplicação é um homomorfismo de grupos abstratos. De fato, sejam $(\lambda, A)$ e $\left(\lambda^{\prime}, A^{\prime}\right)$ elementos de $\mathbb{R}^{+} \times S l(2, \mathbb{R})$. Então temos $\phi\left((\lambda, A) \cdot\left(\lambda^{\prime}, A^{\prime}\right)\right)=\phi\left(\lambda \lambda^{\prime}, A A^{\prime}\right)=$ $\left(\lambda \lambda^{\prime}\right)\left(A A^{\prime}\right)=\lambda^{\prime} \lambda \cdot A A^{\prime}=\phi(\lambda, A) \cdot \phi\left(\lambda^{\prime}, A^{\prime}\right)$. Seja $(\lambda, A) \in \mathbb{R}^{+} \times S l(2, \mathbb{R})$ tal que $(\lambda, A)=I$. Então $\lambda \cdot A=I$, e assim $\operatorname{det} A=\frac{1}{\lambda^{2}}, \operatorname{logo} \lambda=1$ e $A=I$. Logo o homomorfismo em questão é injetor. Seja $B$ uma matriz em $G l^{+}(2, \mathbb{R})$ qualquer com determinante $d>0$. Seja $A$ a matriz em $S l(2, \mathbb{R})$ dada por $\frac{1}{\sqrt{d}} B$. Então para o elemento $(\sqrt{d}, A)$ de $\mathbb{R}^{+} \times S l(2, \mathbb{R})$ temos que $\phi(\sqrt{d}, A)=\sqrt{d} \cdot A=B$; portanto tal homomorfismo é sobrejetor. Queremos mostrar que mais que um homomorfismo, $\phi$ é um homeomorfismo. Lembremos que se $G$ e $G^{\prime}$ são dois grupos topológicos localmente compactos e se $G$ possui base enumerável, então um homomorfismo bijetor de $G$ em $G^{\prime}$ é, mais do que isso, um homeomorfismo. Como todo grupo de Lie é localmente compacto e possui base enumerável, segue que $\phi: \mathbb{R}^{+} \times S l(2, \mathbb{R}) \rightarrow G l^{+}(2, \mathbb{R})$ é um homeomorfismo. Sabendo que $\mathrm{Gl}^{+}(2, \mathbb{R})$ é conexo (é uma componente conexa de $G l(2, \mathbb{R})$, veja [23]), segue que $\mathbb{R}^{+} \times S l(2, \mathbb{R})$ é conexo. Como $S l(2, \mathbb{R})$ é imagem contínua de $\mathbb{R}^{+} \times S l(2, \mathbb{R})$, temos finalmente que $S l(2, \mathbb{R})$ é conexo.

Para finalizar, verifiquemos que $S l(2, \mathbb{R})$ é um grupo de Lie simples. Um grupo de Lie $G$ é simples se sua álgebra de Lie é simples, ou seja, é não abeliana e não possui ideais próprios. Suponhamos por absurdo que $\mathcal{S} l(2, \mathbb{R})$ não é simples e que possui um ideal $\mathcal{H}$ de dimensão 2. Pelas expressões encontradas do colchete em $\left\{b_{1}, b_{2}, b_{3}\right\}$ temos que $\mathcal{G}=[\mathcal{G}, \mathcal{G}]$. Vamos agora escolher uma base $\left\{c_{1}, c_{2}, c_{3}\right\}$ de $\mathcal{G}$ tal que $c_{1}$ e $c_{2}$ pertencem a $\mathcal{H}$ mas $c_{3}$ não pertence a $\mathcal{H}$. Então temos que $\left[c_{1}, c_{2}\right] \in \mathcal{H},\left[c_{1}, c_{3}\right] \in \mathcal{H}$ e $\left[c_{2}, c_{3}\right] \in \mathcal{H}$ pois $\mathcal{H}$ é um ideal em $\mathcal{G}$. Portanto $[\mathcal{G}, \mathcal{G}] \subset \mathcal{H}$, e assim $\mathcal{G}=[\mathcal{G}, \mathcal{G}] \subset \mathcal{H}$ e $\mathcal{G}=\mathcal{H}$. Analogamente mostramos que $\mathcal{G}$ não pode possuir ideais de dimensão 1 , do que segue que $\mathcal{G}$ é uma álgebra simples. 


\section{$5.2 \mathrm{SU}(2)$ e $\mathrm{SO}(3)$}

O conjunto $U(2)$ é formado pelas matrizes $2 \times 2$ complexas unitárias, isto é, $U(2)=\{A \in$ $\left.G l(2, \mathbb{C}) ; A A^{*}=I\right\}$, onde $A^{*}$ denota o transposto conjugado de $A$. Não é difícil verificar que este conjunto é um subgrupo abstrato de $G l(2, \mathbb{C})$. Se $A, B \in U(2)$, então note que $(A B)(A B)^{*}=$ $A B\left(B^{*} A^{*}\right)=A\left(B B^{*}\right) A^{*}=A I A^{*}=A A^{*}=I$ e $\left(A^{-1}\right)\left(A^{-1}\right)^{*}=\left(A^{-1}\right)\left(A^{*}\right)^{-1}=\left(A^{*} A\right)^{-1}=$ $I^{-1}=I$. Agora seja $A=\left(a_{i j}\right)_{i, j}$ pertencente a $U(2)$. Consideremos as seguintes funções:

$$
f_{i j}(A)=a_{i 1} \bar{a}_{j 1}+a_{i 2} \bar{a}_{j 2}=\delta_{i j}
$$

para $1 \leq i, j \leq 1,2$. Notemos que podemos representar $U(2)$ como:

$$
U(2)=\left\{A \in G l(2, \mathbb{C}) ; \phi_{i}=0, i=1,2,3,4\right\}
$$

onde $\phi_{1}=\Re\left(f_{12}\right), \phi_{2}=\Im\left(f_{12}\right), \phi_{3}=\Re\left(f_{11}\right)-1$ e $\phi_{4}=\Re\left(f_{22}\right)-1$. Não é difícil verificar que $\left(d \phi_{1}\right)_{p}, \cdots,\left(d \phi_{4}\right)_{p}$ são linearmente independentes para todo elemento $p \in U(2)$. Isto implica que $U(2)$ é uma subvariedade fechada mergulhada de $G l(2, \mathbb{C})$ de dimensão 4. Em particular as operações de grupos de Lie de $G l(2, \mathbb{C})$, quando restritas a $U(2)$, continuam diferenciáveis. Portanto $U(2)$ é subgrupo de Lie de $G l(2, \mathbb{C})$ de dimensão 4. A sua álgebra de Lie é dada pelo espaço tangente à identidade. Então seja $A(t)$ uma curva em $U(2)$ que passa pela identidade em $t=0$. Temos que $A(t) A(t)^{*}=I$ para todo $t$, logo $\left.\frac{d}{d t}\right|_{t=0} A(t) A(t)^{*}=0$ e a primeira parcela da igualdade é igual a $A^{\prime}(0) A(0)+A(0) A^{\prime}(0)^{*}=A^{\prime}(0)+A^{\prime}(0)^{*}$. Portanto o espaço tangente $T_{I} U(2)$, que é a álgebra de Lie de $U(2)$, é dado por $\left\{A \in M(2, \mathbb{C}) ; A+A^{*}=0\right\}$.

O conjunto $S U(2)$ é formado pelas matrizes $2 \times 2$ complexas unitárias e com determinante igual a 1 , ou seja, $S U(2)=\left\{A \in M_{2 \times 2}(\mathbb{C}) ; A A^{*}=I\right.$ e $\left.\operatorname{det} A=1\right\}$. Consideremos a aplicação $g: U(2) \rightarrow S^{1}$ de $U(2)$ na esfera unitária $S^{1}$ dada pelo determinante, isto é, $g(A)=\operatorname{det} A$. Esta aplicação é um homomorfismo de grupos de Lie pois é diferenciável e $g(A \cdot B)=\operatorname{det}(A \cdot B)=$ $\operatorname{det}(A) \operatorname{det}(B)=g(A) \cdot g(B)$. O kernel deste homomorfismo é exatamente $S U(2)$. Portanto $S U(2)$ é subgrupo de Lie fechado de $U(2)$. Seja $A \in S U(2)$ da forma $\left(\begin{array}{ll}\alpha & \beta \\ \gamma & \delta\end{array}\right)$. Temos que $A A^{*}=I$, portanto:

$$
\begin{gathered}
\left(\begin{array}{cc}
\alpha & \beta \\
\gamma & \delta
\end{array}\right)\left(\begin{array}{cc}
\bar{\alpha} & \bar{\gamma} \\
\bar{\beta} & \bar{\delta}
\end{array}\right)=\left(\begin{array}{ll}
1 & 0 \\
0 & 1
\end{array}\right) \\
\left(\begin{array}{cc}
|\alpha|^{2}+|\beta|^{2} & \alpha \bar{\gamma}+\beta \bar{\delta} \\
\gamma \bar{\alpha}+\delta \bar{\beta} & |\gamma|^{2}+|\delta|^{2}
\end{array}\right)=\left(\begin{array}{ll}
1 & 0 \\
0 & 1
\end{array}\right) .
\end{gathered}
$$

Extraímos disso que $|\alpha|^{2}+|\beta|^{2}=1, \alpha \bar{\gamma}+\beta \bar{\delta}=0, \gamma \bar{\alpha}+\delta \bar{\beta}=0,|\gamma|^{2}+|\delta|^{2}=1$ e também $\alpha \delta-\gamma \beta=1$. Multiplicando esta última relação por $\bar{\gamma}$, por exemplo, encontramos que $\beta=-\bar{\gamma}$. 
Fazendo multiplicações análogas podemos encontrar outras relações de maneira que a matriz $A$ tem a forma $\left(\begin{array}{cc}\alpha & \beta \\ -\bar{\beta} & \bar{\alpha}\end{array}\right)$ e $S U(2)=\left\{\left(\begin{array}{cc}\alpha & \beta \\ -\bar{\beta} & \bar{\alpha}\end{array}\right): \alpha, \beta \in \mathbb{C} ;|\alpha|^{2}+|\beta|^{2}=1\right\}$. Existe um difeomorfismo entre $S U(2)$ e a esfera unitária $\mathbf{S}^{3}$. A esfera pode ser representada pelo conjunto $\left\{(\alpha, \beta) \in \mathbb{C}^{2} ;|\alpha|^{2}+|\beta|^{2}=1\right\}$ e o difeormorfismo é dado por:

$$
S U(2) \ni\left(\begin{array}{cc}
\alpha & \beta \\
-\bar{\beta} & \bar{\alpha}
\end{array}\right) \mapsto(\alpha, \beta) \in \mathbf{S}^{3}
$$

Portanto $S U(2)$ é um grupo de Lie (pelo que vimos antes) de dimensão três simplesmente conexo e compacto, pelo difeomorfismo citado.

Sua álgebra é dada pelo kernel da diferencial do homomorfismo $g$ na identidade. Seja $Z=$ $\left(z_{i j}\right)$ uma matriz em $U(2)$ da forma $\left(\begin{array}{ll}z_{11} & z_{12} \\ z_{21} & z_{22}\end{array}\right)$. Temos que $g(Z)=\operatorname{det} Z=z_{11} z_{22}-z_{12} z_{21} \mathrm{e}$ portanto $\frac{\partial \text { det }}{\partial z_{11}}(Z)=z_{22}$, e assim por diante. Assim, a diferencial de $g$ na identidade é exatamente o traço de $Z$ e a álgebra de Lie de $S U(2)$, que iremos denotar por $\mathcal{S U}(2)$, é o conjunto das matrizes $Z$ pertencentes à álgebra de Lie de $U(2)$ tais que $\operatorname{tr} Z=0$. Fazendo alguns cálculos com essas condições conseguimos encontrar uma maneira mais útil de escrever o conjunto $\mathcal{S U}(2)$ :

$$
\mathcal{S U}(2)=\left\{\left(\begin{array}{cc}
-i t & z \\
-\bar{z} & i t
\end{array}\right) ; t \in \mathbb{R}, z \in \mathbb{C}\right\}
$$

O conjunto $\left\{e_{1}=\frac{1}{2}\left(\begin{array}{cc}i & 0 \\ 0 & -i\end{array}\right), e_{2}=\frac{1}{2}\left(\begin{array}{cc}0 & 1 \\ -1 & 0\end{array}\right), e_{2}=\frac{1}{2}\left(\begin{array}{cc}0 & i \\ i & 0\end{array}\right)\right\}$ é uma base para $\mathcal{S U}(2)$ tal que:

$$
\begin{aligned}
& {\left[e_{1}, e_{2}\right]=e_{3},} \\
& {\left[e_{3}, e_{1}\right]=e_{2},} \\
& {\left[e_{2}, e_{3}\right]=e_{1} .}
\end{aligned}
$$

Precisamos verificar que tal grupo é unimodular. Para encontrar $a d_{x}\left(e_{1}\right)$, fazemos a operação colchete, onde não é difícil encontrar que $a d_{x}\left(e_{1}\right)=-a_{2} e_{3}+a_{3} e_{2}$, onde $x=\sum_{i} a_{i} e_{i}$ e $a_{i} \in \mathbb{C}$. Analogamente podemos encontrar $a d_{x}\left(e_{2}\right)$ e $a d_{x}\left(e_{3}\right)$ e mostrar que $a_{22}$ e $a_{33}$ são iguais a zero. Logo o traço de $a d_{x}$ é identicamente nulo, para qualquer $x \in \mathcal{S U}(2)$ e $S U(2)$ é unimodular. Podemos mostrar que $S U(2)$ é simples exatemente como fizemos em 5.1 .

$\mathrm{O}$ conjunto $S O(3)$ é definido por $\left\{A \in M(3, \mathbb{R}) ; \operatorname{det} A=1\right.$ e $\left.A A^{t}=I\right\}$. Consideremos também $G l^{+}(3, \mathbb{R})=\{A \in M(3, \mathbb{R}) ; \operatorname{det}(A)>0\}$, que é um subconjunto aberto de $\mathbb{R}^{9}$. Definimos 
$f: G l^{+}(3, \mathbb{R}) \rightarrow \operatorname{Sim}(3, \mathbb{R})$ dada por $f(A)=A^{t} A$. Temos $S O(3)=f^{-1}(\{I\})$ e podemos mostrar que a diferencial $d f_{A}$ é sobrejetora para toda matriz $A \in S O(3)$. De fato:

$$
\begin{gathered}
d f_{A}(H)=\lim _{t \rightarrow 0} \frac{f(A+t H)-f(A)}{t} \\
=\lim _{t \rightarrow 0} \frac{(A+t H)^{t}(A+t H)-A^{t} A}{t}=A^{t} H+H^{t} A .
\end{gathered}
$$

Seja $B$ uma matriz simétrica qualquer. Então é claro que $\frac{A B}{2}$ pertence a $T_{A}(G l(3, \mathbb{R}))$ e de fato temos $d f_{A}\left(\frac{A B}{2}\right)=B$. Portanto $d f_{A}$ é sobrejetora para qualquer $A \in S O(2)$ e segue que $S O(3)$ é subvariedade mergulhada (e portanto é um subgrupo de Lie) de $G l(3, \mathbb{R})$ de dimensão 3.

Como tal conjunto é imagem inversa de um fechado por uma função contínua, segue que $S O(3)$ é um subgrupo de Lie fechado de $G l(3, \mathbb{R})$. A condição de que $A A^{t}=I$ implica que a soma dos quadrados das entradas de cada linha de $A$ é igual a 1 , do que concluimos que $S O(3)$ é compacto. Portanto tal conjunto é um grupo de Lie compacto de dimensão três. A álgebra de Lie de $S O(3)$, denotada por $\mathcal{S O}(3)$, constitui-se das matrizes $3 \times 3$ reais tais que $A+A^{t}=0$ e $\operatorname{tr} A=0$. Não é difícil concluir que o conjunto

$$
\left\{e_{1}=\frac{1}{2}\left(\begin{array}{ccc}
0 & -1 & 0 \\
1 & 0 & 0 \\
0 & 0 & 0
\end{array}\right), e_{2}=\frac{1}{2}\left(\begin{array}{ccc}
0 & 0 & -1 \\
0 & 0 & 0 \\
1 & 0 & 0
\end{array}\right), e_{3}=\frac{1}{2}\left(\begin{array}{ccc}
0 & 0 & 0 \\
0 & 0 & -1 \\
0 & 1 & 0
\end{array}\right)\right\}
$$

é uma base para $\mathcal{S O}(3)$ tal que:

$$
\begin{aligned}
& {\left[e_{1}, e_{2}\right]=e_{3},} \\
& {\left[e_{3}, e_{1}\right]=e_{2},} \\
& {\left[e_{2}, e_{3}\right]=e_{1} .}
\end{aligned}
$$

Estas relações de colchete mostram que a álgebra $\mathcal{S O}(3)$ e $\mathcal{S U}(2)$ são isomorfas, o que implica. que $\mathcal{S O}(3)$ é simples e unimodular.

Para finalizar, sabemos que toda rotação no espaço euclidiano pode ser representada por uma matriz pertencente a $S O(3)$. Toda rotação está determinada pelo seu eixo de rotação e pelo ângulo $\theta \operatorname{com} 0 \leq \theta \leq \pi$ pelo qual ocorre a rotação do espaço através do eixo. Se escolhermos a direção do eixo como sendo aquela cuja rotação por $\theta$ é no sentido horário, então podemos dizer que os elementos de $S O(3)$ estão todos determinados por um vetor que será aquele correspondente ao eixo escolhido como antes e pela sua norma, que será dada por $\theta$. Olhemos então para a bola fechada de raio $\pi$ no espaço euclidiano. Então a cada ponto de $S O(3)$ temos um ponto da bola, mas note que esta correspondência não é injetiva pois os pontos antípodas da bola correspondem a rotações de ângulo $\pi$ através do mesmo eixo, mas em direções opostas (e estas rotações definem 
um mesmo elemento em $S O(3))$. Ao identificarmos estes pontos antípodas, obtemos um espaço topológico homemorfo ao espaço $S O(3)$. Na realidade este espaço é uma variedade diferenciável difeomorfa a $S O(3)$ e também difeomorfa ao espaço projetivo real de dimensão três. Esta é uma maneira de ver que $S O(3)$ é conexo (mas não é simplesmente conexo).

\section{$\mathbf{5 . 3} O(1,2)$}

Sejam $x, y$ dois vetores em $\mathbb{R}^{n}$. A operação $\ll x, y \gg=-x_{1} y_{1}+x_{2} y_{2}+\cdots+x_{n} y_{n}$ é uma forma bilinear simétrica não-degenerada indefinida em $\mathbb{R}^{n}$, e o espaço $\mathbb{R}^{n}$ juntamente com esse semiproduto é chamado espaço Lorentziano de dimensão $n$ e é denotado por $\mathbb{R}^{1, n-1}$. Dizemos que uma base $\left\{u_{1}, \cdots, u_{n}\right\}$ de $\mathbb{R}^{n}$ é ortonormal segundo Lorentz se $\ll u_{1}, u_{1} \gg=-1 \mathrm{e} \ll u_{i}, u_{j} \gg=\delta_{i j}$

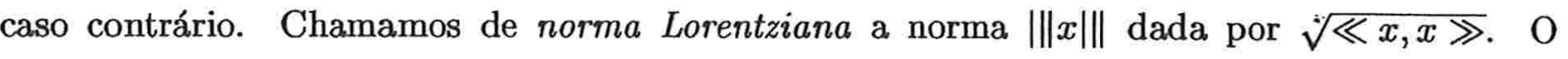
conjunto dos $x$ pertencentes a $\mathbb{R}^{n}$ tais que $\left|\|x \mid\|=0\right.$ forma um cone, chamado de cone-luz de $\mathbb{R}^{n}$. Quando $|\|x \mid\|=0$ dizemos que $x$ é um vetor tipo-luz, se $|\|x \mid\|>0$ dizemos que ele é tipo-espaço e se $|\|x \mid\|<0$ dizemos que ele é tipo-tempo.

Uma aplicação $L: \mathbb{R}^{n} \rightarrow \mathbb{R}^{n}$ tal que $\ll L(x), L(y) \gg=\ll x, y \gg$ para todos $x, y \in \mathbb{R}^{n}$ é chamada de transformação de Lorentz. Uma matriz $A$ real $n \times n$ é chamada de matriz Lorentziana se e somente se a transformação linear associada a $A, A: \mathbb{R}^{n} \rightarrow \mathbb{R}^{n}$ dada por $A(x)=A \cdot x$ é uma transformação de Lorentz. O conjunto das matrizes Lorentzianas com a operação usual forma um grupo geralmente denotado por $O(1, n-1)$ e chamado de grupo de Lorentz. Consideremos agora, por simplicidade e de acordo com nossos interesses, o caso $n=3$.

Podemos mostrar que se $A$ pertence a $O(1,2)$, então ela satisfaz $A^{t} J A=A J A^{t}=J$, onde $J=$ $\left(\begin{array}{ccc}-1 & 0 & 0 \\ 0 & 1 & 0 \\ 0 & 0 & 1\end{array}\right)$

De fato, por hipótese $A$ é uma matriz de Lorentz. Portanto, a transformação $A: \mathbb{R}^{3} \rightarrow \mathbb{R}^{3}$ dada por $A(x)=A \cdot x$ preserva o produto interno $\ll \cdot, \cdot \gg$. Lembremos que, como no caso do produto interno usual do $\mathbb{R}^{3}$, a transformação $A$ é de Lorentz se e somente se é linear e $\left\{A\left(e_{1}\right), A\left(e_{2}\right), A\left(e_{3}\right)\right\}$ é uma base ortonormal segundo Lorentz, onde $\left\{e_{1}, e_{2}, e_{3}\right\}$ é base canônica do $\mathbb{R}^{3}$. Isto implica evidentemente que as colunas de $A$ formam uma base ortonormal segundo Lorentz de $\mathbb{R}^{3}$. Portanto vale:

$$
\left(\begin{array}{lll}
\ll A\left(e_{1}\right), A\left(e_{1}\right) \gg & \ll A\left(e_{1}\right), A\left(e_{2}\right) \gg & \ll A\left(e_{1}\right), A\left(e_{3}\right) \gg \\
\ll A\left(e_{2}\right), A\left(e_{1}\right) \gg & \ll A\left(e_{2}\right), A\left(e_{2}\right) \gg & \ll A\left(e_{2}\right), A\left(e_{3}\right) \gg \\
\ll A\left(e_{3}\right), A\left(e_{1}\right) \gg & \ll A\left(e_{3}\right), A\left(e_{2}\right) \gg & \ll A\left(e_{3}\right), A\left(e_{3}\right) \gg
\end{array}\right)=\left(\begin{array}{ccc}
-1 & 0 & 0 \\
0 & 1 & 0 \\
0 & 0 & 1
\end{array}\right)
$$

e a primeira matriz é exatamente igual a $A^{t} J A$, do que segue a afirmação inicial. Se $A$ pertence 
a. $O(1,2)$ então $A^{t} J A=J$, assim é fácil observar que $(\operatorname{det} A)^{2}=1$ e $\operatorname{det} A= \pm 1$. Podemos definir precisamente o conjunto $O(1,2)$ como:

$$
O(1,2)=\left\{A \in G l(3, \mathbb{R}) ; A^{t} J A=J\right\}
$$

Este conjunto é subgrupo abstrato de $G l(3, \mathbb{R})$. De fato, sejam $A, B$ pertencentes a $O(1,2)$. Então:

$$
(A B)^{t} J(A B)=\left(B^{t} A^{t}\right) J(A B)=B^{t}\left(A^{t} J A\right) B=B^{t} J B=J
$$

portanto $A B$ pertence a. $O(1,2)$. Seja $A \in O(1,2)$. Então:

$$
\left(A^{-1}\right)^{t} J A^{-1}=\left(A^{t}\right)^{-1} J A^{-1}=\left((A J) A^{t}\right)^{-1}=J^{-1}=J,
$$

logo $A^{-1}$ pertence a $O(1,2)$. Considere a aplicação $f: G l(3, \mathbb{R}) \rightarrow \operatorname{Sim}(3, \mathbb{R})$ dada por $f(A)=$ $A^{t} J A$. Então esta aplicação é $C^{\infty}$ e $f^{-1}(\{J\})=O(1,2)$. Se mostrarmos que $J$ é valor regular de $f$, então $f^{-1}(\{J\})=O(1,2)$ admite estrutura de variedade diferenciável que a torna subvariedade mergulhada de $G l(3, \mathbb{R})$ de dimensão 3. Para isso, precisamos mostrar que $f$ é uma submersão em todo ponto $P \in f^{-1}(J)$, ou seja, que a diferencial $d f_{P}$ é sobrejetora para todo $P \in f^{-1}(J)$. A diferencial é dada por:

$$
\begin{gathered}
d f_{P}(X)=\lim _{s \rightarrow 0} \frac{f(P+s X)-f(P)}{s} \\
=\lim _{s \rightarrow 0} \frac{(P+s X)^{t} J(P+s X)-P^{t} J P}{s} \\
=\lim _{s \rightarrow 0} \frac{\left(P^{t}+s X^{t}\right) J(P+s X)-J}{s} \\
=\lim _{s \rightarrow 0} \frac{\left(P^{t} J P+s X^{t} J P+s P^{t} J X+t^{2} X^{t} J X-J\right.}{s} \\
=X^{t} J P+P^{t} J X .
\end{gathered}
$$

Seja $C \in \operatorname{Sim}(3, \mathbb{R})$, tomemos $B=\frac{1}{2} J\left(P^{-1}\right)^{t} C$ (observe que $\operatorname{det} P= \pm 1$ ). Então:

$$
\begin{gathered}
d f_{P}(B)=\left(\frac{1}{2} J\left(P^{-1}\right)^{t} C\right)^{t} J P+P^{t} J\left(\frac{1}{2} J\left(P^{-1}\right)^{t} C\right)= \\
\frac{1}{2}\left(C\left(\left(P^{-1}\right)^{t}\right)^{t} J J P+P^{t}\left(P^{t}\right)^{-1} C\right)=C .
\end{gathered}
$$

Logo $f$ é submersão em todo ponto de $f^{-1}(J)$, como queríamos. Portanto, $O(1,2)$ é subvariedade mergulhada de $G L(3, \mathbb{R})$ de dimensão três e por isso as operações de grupo de Lie de $G l(3, \mathbb{R})$, $(A, B) \mapsto A \cdot B$ e $A \mapsto A^{-1}$ quando restritas à $O(1,2)$ continuam diferenciáveis. Mostramos assim que $O(1,2)$ é um grupo de Lie de dimensão três. 
Vamos encontrar sua álgebra de Lie. Para tanto achemos o espaço tangente à identidade $T_{I} O(1,2)$ através do kernel da diferencial da função $f$ na identidade $I$, onde $X \in M(3, \mathbb{R})$, que é dada por $d f_{I}(X)=X^{t} J+J X$. A álgebra de Lie de $O(1,2)$, que iremos denotar por $\mathcal{O}(1,2)$, é o conjunto:

$$
\mathcal{O}(1,2)=\left\{A \in M(3, \mathbb{R}) ; A J+J A^{t}=0\right\}
$$

Não é difícil fazer uma descrição matricial com estas condições e obter que:

$$
\mathcal{O}(1,2)=\left\{A \in M(3, \mathbb{R}) ; A=\left(\begin{array}{cc}
a & x \\
x^{t} & B
\end{array}\right)\right\}
$$

onde $a \in \mathbb{R}$ qualquer, $x$ é um vetor linha qualquer e $B$ é uma matriz $2 \times 2$ real anti-simétrica.

O grupo $O(1,2)$ não é conexo. Ele possui quatro componentes conexas, dependendo do fato da matriz preservar ou reverter a orientação do tempo ou espaço. Mais claramente, se $A \in O(1,2)$ é da forma $\left(\begin{array}{cc}a_{t} & b \\ c & D\end{array}\right)$, onde $a_{t} \in \mathbb{R}$ e $D$ é uma matriz $2 \times 2$ real, dizemos que $A$ preserva (reverte) a orientação do tempo se $a_{t}>0\left(a_{t}<0\right)$ e que $A$ preserva (reverte) a orientação do espaço se $\operatorname{det} D>0(\operatorname{det} D<0)$. Temos assim quatro componentes conexas correspondentes à matriz preservar tempo e espaço, preservar tempo mas reverter espaço, e assim por diante. É possível mostrar que a componente conexa na identidade é dada pelo subgrupo de $O(1,2)$ das matrizes com determinante igual a 1 e que preservam a orientação de tempo, veja [18]. Indiquemos este subgrupo por $S O_{0}(1,2)$ e o chamemos de grupo de Lorentz especial. Como $S O_{0}(1,2)$ é a componente conexa na identidade de $O(1,2)$, é um grupo de Lie conexo e sua álgebra de Lie $\mathcal{S O}_{0}(1,2)$ é isomorfa (isomorfismo de álgebras de Lie) à álgebra de Lie de $O(1,2)$. É fácil verificar que o conjunto $\left\{e_{1}=\left(\begin{array}{lll}0 & 1 & 0 \\ 1 & 0 & 0 \\ 0 & 0 & 0\end{array}\right), e_{2}=\left(\begin{array}{lll}0 & 0 & 1 \\ 0 & 0 & 0 \\ 1 & 0 & 0\end{array}\right), e_{3}=\left(\begin{array}{ccc}0 & 0 & 0 \\ 0 & 0 & 1 \\ 0 & -1 & 0\end{array}\right)\right\}$ forma uma base para $\mathcal{S O}_{0}(1,2)$ e vale que:

$$
\begin{gathered}
{\left[e_{1}, e_{2}\right]=e_{3},} \\
{\left[e_{3}, e_{1}\right]=-e_{2},} \\
{\left[e_{2}, e_{3}\right]=-e_{1} .}
\end{gathered}
$$

Dessas relações concluimos que as álgebras $\mathcal{S O}_{0}(1,2)$ e $\mathcal{S L}(2, \mathbb{R})$ são isomorfas e portanto $\mathcal{S O}_{0}(1,2)$ é unimodular e simples. 


\section{$5.4 E(2)$}

$E(2)$ é definido como sendo o conjunto dos movimentos rígidos, ou isometrias, do plano euclidiano. Podemos representar movimentos rígidos que preservam orientação pela ação natural do conjunto $S O(2)$ em vetores de $\mathbb{R}^{2}$, seguida de uma translação. Podemos então definir $E(2)$ como sendo o produto semidireto $S O(2) \bowtie \mathbb{R}^{2}$. Então $E(2)=\left(S O(2) \times \mathbb{R}^{2}\right.$, o $)$ onde $(X, \xi) \circ$ $(Y, \eta)=(X Y, X \eta+\xi)$. Com essa operação $E(2)$ é um grupo de Lie (conexo) de dimensão três. Como conjunto de matrizes, podemos representar $E(2)$ como o conjunto das matrizes reais $3 \times 3$ da forma $\left(\begin{array}{cc}A & b \\ 0 & 1\end{array}\right)$ onde $A$ pertence a $S O(2)$ e $b$ é um vetor em $\mathbb{R}^{2}$. Sua álgebra é dada pelo produto semi direto das álgebras de $S O(2)$ e $\mathbb{R}^{2}$ que como conjunto de matrizes pode ser escrita como:

$$
\mathcal{E}(2)=\left\{X \in M\left(3, \mathbb{R}^{3}\right) ; X=\left(\begin{array}{cc}
A & b \\
0 & 0
\end{array}\right), A+A^{t}=0, b \in \mathbb{R}^{2}\right\} .
$$

Não é difícil calcular uma base para esse espaço, que é dada por

$$
\left\{e_{1}=\left(\begin{array}{ccc}
0 & -1 & 0 \\
1 & 0 & 0 \\
0 & 0 & 0
\end{array}\right), e_{2}=\left(\begin{array}{ccc}
0 & 0 & 1 \\
0 & 0 & 0 \\
0 & 0 & 0
\end{array}\right), e_{3}=\left(\begin{array}{lll}
0 & 0 & 0 \\
0 & 0 & 1 \\
0 & 0 & 0
\end{array}\right)\right\}
$$

e que satisfaz:

$$
\begin{aligned}
& {\left[e_{1}, e_{2}\right]=e_{3},} \\
& {\left[e_{2}, e_{3}\right]=0,} \\
& {\left[e_{3}, e_{1}\right]=e_{2} .}
\end{aligned}
$$

Vamos verificar que esta álgebra é unimodular. Para isso precisamos verificar que $\operatorname{trad}_{x} \equiv 0$ para todo $x \in \mathcal{E}(2)$. Não é difícil ver que para $x \in \mathcal{E}(2)$, temos $a d_{x}\left(e_{1}\right)=\left[x, e_{1}\right]=b_{1} e_{2}+b_{2} e_{3}$ onde $b_{1}, b_{2} \in \mathbb{R}$. O mesmo pode ser feito para $a d_{x}\left(e_{2}\right)$ e $a d_{x}\left(e_{3}\right)$ para concluir que a álgebra é unimodular. Portanto o grupo euclidiano $E(2)$ é unimodular. Podemos mostrar que $E(2)$ é solúvel. Um grupo de Lie é solúvel se sua álgebra de Lie é solúvel. Lembrando que se $\mathcal{G}$ é uma álgebra de Lie, então a série derivada de $\mathcal{G}$ é construida tomando a sequência de subalgebras de $\mathcal{G}$ dada por $\mathcal{G} \supseteq[\mathcal{G}, \mathcal{G}] \supseteq[[\mathcal{G}, \mathcal{G}],[\mathcal{G}, \mathcal{G}]] \supseteq \cdots$ e dizemos que $\mathcal{G}$ é solúvel se algum termo dessa sequência é nulo. Sejam $x, y$ elementos quaisquer de $\mathcal{E}(2)$. Então temos $x=a_{1} e_{1}+a_{2} e_{2}+a_{3} e_{3} \mathrm{e}$ $y=b_{1} e_{1}+b_{2} e_{2}+b_{3} e_{3}$ e calculando diretamente temos que $[x, y]=\left(a_{1} b_{3}-a_{3} b_{1}\right) e_{2}+\left(a_{2} b_{1}-a_{1} b_{2}\right) e_{3}$ do que segue imediatamente que $[[x, y],[x, y]]=0$, pois $\left[e_{2}, e_{3}\right]=0$. Logo a álgebra é solúvel e $E(2)$ é solúvel. 


\section{$\mathbf{5 . 5} E(1,1)$}

O conjunto $E(1,1)$, também chamado de grupo de Poincaré, é formado pelos movimentos rígidos do plano de Minkowski. O plano de Minskowski é uma variedade pseudo-Riemanniana, isto é, uma variedade diferenciável com uma dada métrica pseudo-Riemanniana. Uma métrica pseudo-Riemanniana é uma escolha, para cada ponto $p$ da variedade, de uma forma bilinear simétrica não degenerada < > > (que não precisa ser, necessariamente, definida positiva) no espaço tangente à variedade no dado ponto. No caso do plano de Minkowski, tomamos $\mathbb{R}^{2}$ onde $\frac{\partial}{\partial x_{1}}$ é identificado com $e_{1}=(1,0)$ e $\frac{\partial}{\partial x_{2}} \operatorname{com} e_{2}=(0,1)$. A métrica é dada por $\left\langle e_{1}, e_{1}\right\rangle=1$, $\left.\left.<e_{2}, e_{2}\right\rangle=-1 \mathrm{e}<e_{i}, e_{j}\right\rangle=0$ caso contrário. Se $x$ pertence ao plano de Minkowski, então a translação $\phi(v)=x+v$ é uma isometria deste conjunto. Na realidade, todo movimento rígido de $E(1,1)$ é dado por $\phi \circ \tau$, onde $\tau$ é uma rotação hiperbólica representada por uma matriz do grupo de Lorentz especial $S O_{0}(1,1)$. O conjunto $E(1,1)$ pode ser escrito como um produto semidireto $S O_{0}(1,1) \bowtie \mathbb{R}^{2}$ e isso mostra que $E(1,1)$ é um grupo de Lie conexo de dimensão 3. Sua álgebra é dada pelo produto semi direto das álgebras de $S O_{0}(1,1)$ e $\mathbb{R}^{2}$ que como conjunto de matrizes, pode ser escrita como $\mathcal{E}(1,1)=\left\{X \in M(3, \mathbb{R}) ; X=\left(\begin{array}{cc}A & b \\ 0 & 0\end{array}\right), A \in M(2, \mathbb{R}), A J+J A^{t}=0, b \in\right.$ $\left.\mathbb{R}^{2}\right\}$. Não é difícil ver que o conjunto:

$$
\left\{e_{1}=\left(\begin{array}{lll}
0 & 1 & 0 \\
1 & 0 & 0 \\
0 & 0 & 0
\end{array}\right), e_{2}=\left(\begin{array}{lll}
0 & 0 & 1 \\
0 & 0 & 0 \\
0 & 0 & 0
\end{array}\right), e_{3}=\left(\begin{array}{lll}
0 & 0 & 0 \\
0 & 0 & 1 \\
0 & 0 & 0
\end{array}\right)\right\}
$$

forma uma base para o espaço $\mathcal{E}(1,1)$. Um cálculo com esta base mostra que:

$$
\begin{gathered}
{\left[e_{1}, e_{2}\right]=e_{3},} \\
{\left[e_{2}, e_{3}\right]=0,} \\
{\left[e_{3}, e_{1}\right]=-e_{2} .}
\end{gathered}
$$

Analogamente ao caso de $E(2)$ podemos mostrar que $[[x, y],[x, y]]=0$ para todo $x, y \in \mathcal{E}(1,1)$. Portanto $\mathcal{E}(1,1)$ é uma álgebra solúvel e assim $E(1,1)$ é um grupo de Lie solúvel. 


\subsection{Grupo de Heisenberg}

O grupo de Heisenberg, que denotaremos por $\mathbf{H}$ é formado pelas matrizes $3 \times 3$ reais da forma $\left(\begin{array}{lll}1 & x & z \\ 0 & 1 & y \\ 0 & 0 & 1\end{array}\right)$. Podemos identificar $\mathbf{H}$ com $\mathbb{R}^{3}$ através de:

$$
\left(\begin{array}{ccc}
1 & x & z \\
0 & 1 & y \\
0 & 0 & 1
\end{array}\right) \mapsto(x, y, z)
$$

Dessa maneira, $\mathbf{H}$ herda uma estrutura de variedade de $\mathbb{R}^{3}$, de maneira natural. Além disso $\mathbf{H}$ é subgrupo abstrato de $S l(3, \mathbb{R})$, de fato, sejam $A=\left(\begin{array}{lll}1 & x & z \\ 0 & 1 & y \\ 0 & 0 & 1\end{array}\right)$ e $B=\left(\begin{array}{lll}1 & x^{\prime} & z^{\prime} \\ 0 & 1 & y^{\prime} \\ 0 & 0 & 1\end{array}\right)$ dois elementos de $\mathbf{H}$, então:

$$
\begin{gathered}
A \cdot B=\left(\begin{array}{ccc}
1 & x+x^{\prime} & z+z^{\prime}+x y^{\prime} \\
0 & 1 & y+y^{\prime} \\
0 & 0 & 1
\end{array}\right) \in \mathbf{H} \\
A^{-1}=\left(\begin{array}{ccc}
1 & -x & -z+x y \\
0 & 1 & -y \\
0 & 0 & 1
\end{array}\right) \in \mathbf{H}
\end{gathered}
$$

Logo este conjunto também possui estrutura de grupo; e também observemos que as aplicações $\mathbb{R}^{3} \times \mathbb{R}^{3} \rightarrow \mathbb{R}^{3}$ dada por $\left((x, y, z),\left(x^{\prime}, y^{\prime}, z^{\prime}\right)\right) \mapsto\left(x+x^{\prime}, y+y^{\prime}, z+z^{\prime}+x y^{\prime}\right)$ e $\mathbb{R}^{3} \rightarrow \mathbb{R}^{3}$ dada por $(x, y, z) \mapsto(-x,-y,-z+x y)$ são $C^{\infty}$. Logo $\mathbf{H}$ é um grupo de Lie de dimensão três. Seja $A(t)$ um subgrupo a um parâmetro de $\mathbf{H}$. Então existe $X \in \mathcal{H}$ tal que $A(t)=\exp t X$, onde $\mathcal{H}$ é a álgebra de Lie de $\mathbf{H}$. Além disso, $\left.\frac{d}{d t}\right|_{t=0} A(t)=X \operatorname{e} \exp t X \in \mathbf{H}$ para todo $t$. Derivando o subgrupo a um parâmetro, temos que $X$ é da forma $\left(\begin{array}{ccc}0 & a & c \\ 0 & 0 & b \\ 0 & 0 & 0\end{array}\right)$ onde $a, b, c$ são reais quaisquer. Podemos então escrever a álgebra de Lie de $\mathbf{H}$ como sendo formada pelas matrizes dessa forma. É imediato concluir que o conjunto $\left\{\left(\begin{array}{lll}0 & 1 & 0 \\ 0 & 0 & 0 \\ 0 & 0 & 0\end{array}\right),\left(\begin{array}{lll}0 & 0 & 1 \\ 0 & 0 & 0 \\ 0 & 0 & 0\end{array}\right),\left(\begin{array}{lll}0 & 0 & 0 \\ 0 & 0 & 1 \\ 0 & 0 & 0\end{array}\right)\right\}$ é uma base de $\mathcal{H}$ tal que: 


$$
\begin{gathered}
{\left[e_{1}, e_{2}\right]=0} \\
{\left[e_{2}, e_{3}\right]=0} \\
{\left[e_{3}, e_{1}\right]=-e_{2} .}
\end{gathered}
$$

Podemos mostrar que $\mathcal{H}$ é uma álgebra nilpotente, isto é, algum termo da série $\mathcal{H} \supseteq[\mathcal{H}, \mathcal{H}] \supseteq$ $[\mathcal{H},[\mathcal{H}, \mathcal{H}]] \supseteq \cdots$ é nulo. De fato, sejam $x, y, z$ elementos quaisquer de $\mathcal{H}$, que podemos expressar como combinações lineares reais dos elementos da base. Então sejam $x=\sum_{i} a_{i} e_{i}, y=\sum_{j} b_{j} e_{j}$ e $z=\sum_{k} c_{k} e_{k}$. Observemos que:

$$
[x,[y, z]]=\left[\sum_{i} a_{i} e_{i},\left[\sum_{j} b_{j} e_{j}, \sum_{k} c_{k} e_{k}\right]\right]=\left[\sum_{i} a_{i} e_{i},\left(b_{1} c_{3}-b_{3} c_{1}\right) e_{2}\right]=0
$$

pois $\left[e_{1}, e_{2}\right]=0$ e $\left[e_{2}, e_{3}\right]=0$. Portanto $[\mathcal{H},[\mathcal{H}, \mathcal{H}]]$ é nulo e $\mathcal{H}$ é nilpotente.

Vimos aqui, desde o exemplo 5.1 até 5.6, exemplos de cada caso de sinais de $\lambda_{1}, \lambda_{2}, \lambda_{3}$, sendo que no total temos 6 casos de sinais. Em [17], J. Milnor organiza cada um destes casos em uma tabela que reproduziremos de uma maneira um pouco diferente aqui:

$\begin{array}{llr}\text { Sinais de } \lambda_{1}, \lambda_{2}, \lambda_{3} & \text { Grupo de Lie associado } & \text { Propriedade do grupo } \\ +,+,+ & S U(2) \text { ou } S O(3) & \text { compacto e simples } \\ +,+,- & S L(2, \mathbb{R}) \text { ou } O(1,2) & \text { simples } \\ +,+, 0 & E(2) & \text { solúvel } \\ +,-, 0 & E(1,1) & \text { solúvel } \\ +, 0,0 & \text { Grupo de Heisenberg } & \text { nilpotente } \\ 0,0,0 & \mathbb{R} \oplus \mathbb{R} \oplus \mathbb{R} & \text { comutativo }\end{array}$

Estudemos agora a curvatura, em específico a curvatura de Ricci destes exemplos da tabela. Utilizaremos principalmente as técnicas desta seção, como o Lema 7. Este é o tema da próxima seção. 


\section{Curvatura em grupos de Lie unimodulares}

Nesta, seção denotaremos os sinais das curvaturas principais de Ricci por $\operatorname{sgn}\left(r\left(e_{1}\right), r\left(e_{2}\right), r\left(e_{3}\right)\right)$.

Em 5.2 mostramos que $S U(2)$ é um grupo de Lie de dimensão três, unimodular, simples, compacto, simplesmente conexo (e portanto possui grupo fundamental finito). Pelo Teorema 1 da Seção 3, SU(2) admite uma métrica invariante à esquerda com todas as curvaturas de Ricci estritamente positivas. Logo, para esta métrica temos $\operatorname{sgn}\left(r\left(e_{1}\right), r\left(e_{2}\right), r\left(e_{3}\right)\right)=(+,+,+)$ e consequentemente a curvatura escalar também é estritamente positiva. Ao mesmo tempo, como $\mathcal{S U}(2)$ é uma álgebra de Lie não comutativa, pelo Teorema 5 da Seção 4, $S U(2)$ possui uma métrica invariante à esquerda de curvatura escalar estritamente negativa.

Em 5.2 encoṇtramos os autovalores $\lambda_{i}=1$, para $i=1,2,3$. Mas pelo que observamos após a demonstração do Lema 7, podemos mudar um pouco a métrica em $S U(2)$ de maneira que, por exemplo, $\lambda_{1}=\lambda_{2}-\lambda_{3} \operatorname{com} \lambda_{2}>\lambda_{3}>0$. De acordo com o Lema 7 , teríamos nesse caso:

$$
\begin{gathered}
r\left(e_{1}\right)=\frac{1}{2}\left(\left(\lambda_{1}\right)^{2}-\left(\lambda_{2}-\lambda_{3}\right)^{2}\right)=0, \\
r\left(e_{2}\right)=\frac{1}{2}\left(\left(\lambda_{2}\right)^{2}-\left(\lambda_{1}-\lambda_{3}\right)^{2}\right)=\frac{1}{2}\left(\left(\lambda_{2}\right)^{2}-\left(\lambda_{2}-\lambda_{3}-\lambda_{3}\right)^{2}\right) \\
=\frac{1}{2}\left(\lambda_{2}^{2}-\lambda_{2}^{2}+4 \lambda_{2} \lambda_{3}-4 \lambda_{3}^{2}\right)=\frac{1}{2}\left(4 \lambda_{3}\left(\lambda_{2}-\lambda_{3}\right)\right)>0, \\
r\left(e_{3}\right)=\frac{1}{2}\left(\left(\lambda_{3}\right)^{2}-\left(\lambda_{2}-\lambda_{1}\right)^{2}\right)=0 .
\end{gathered}
$$

Portanto podemos ter também $\operatorname{sgn}\left(r\left(e_{1}\right), r\left(e_{2}\right), r\left(e_{3}\right)\right)=(0,+, 0)$. Observe que podemos escrever a curvatura escalar em função dos autovalores $\lambda_{i}$. De fato, substituindo as fórmulas encontradas no Lema 7 e simplificando, temos:

$$
\rho=\left(\lambda_{1} \lambda_{2}+\lambda_{2} \lambda_{3}+\lambda_{1} \lambda_{3}\right)-\frac{1}{2}\left(\lambda_{1}^{2}+\lambda_{2}^{2}+\lambda_{3}^{2}\right) .
$$

Podemos perguntar se existem $\lambda_{1}, \lambda_{2}, \lambda_{3}$ estritamente positivos tais que $\rho=0$. Mantendo $\lambda_{2}$, $\lambda_{3}$ constantes, escrevemos uma equação quadrática em $\lambda_{1}$ e a resolvemos. Em $\lambda_{1}$ temos, por exemplo, a seguinte equação:

$$
\rho\left(\lambda_{1}\right)=-\frac{1}{2} \lambda_{1}^{2}+\lambda_{1}\left(\lambda_{2}+\lambda_{3}\right)+\frac{1}{2}\left(\lambda_{3}-\lambda_{2}\right)^{2} .
$$

Resolvendo essa equação encontra-se que $\lambda_{1}=\left(\lambda_{2}+\lambda_{3}\right)+2 \sqrt{\lambda_{2} \lambda_{3}}$ é solução, onde $\lambda_{i}>0$, para $i=1,2,3$. Podemos calcular a curvatura de Ricci para estes valores de $\lambda_{i}$. Não é difícil concluir que teremos os sinais dados por $(+,-,-)$. 
A conclusão final é que em $S U(2)$, dependendo da métrica invariante à esquerda escolhida, teremos os sinais das curvaturas de Ricci $(+,+,+),(+, 0,0)$ ou $(+,-,-)$ e a curvatura escalar pode ser estritamente positiva, estritamente negativa ou nula.

Em contraste, estudemos o grupo de Heisenberg. Vimos em 5.6 que o grupo de Heisenberg é um grupo de Lie conexo de dimensão três, unimodular e nilpotente e encontramos $\lambda_{1}>0$, $\lambda_{2}=\lambda_{3}=0$. Substituindo nas fórmulas encontradas no Lema 7 , temos:

$$
r\left(e_{1}\right)=-r\left(e_{2}\right)=-r\left(e_{3}\right)=-\rho=\frac{\lambda_{1}^{2}}{2}
$$

Portanto, para qualquer métrica invariante à esquerda no grupo de Heisenberg, as curvaturas principais de Ricci têm sinal $\operatorname{sgn}\left(r\left(e_{1}\right), r\left(e_{2}\right), r\left(e_{3}\right)\right)=(+,-,-)$ e a curvatura escalar é sempre estritamente negativa (veja Teorema 2 da Seção 3 ).

Mostramos em 5.5 que o grupo $E(1,1)$ é um grupo de Lie de dimensão três unimodular e solúvel e encontramos $\lambda_{1}=0, \lambda_{2}<0$ e $\lambda_{3}>0$. Utilizando novamente o Lema 7 da Seção 5 , temos, por exemplo, $r\left(e_{1}\right)<0, r\left(e_{2}\right)=-r\left(e_{3}\right)$. Podemos alterar um pouco a métrica de maneira que $\lambda_{1}=0$ e $\lambda_{2}=-\lambda_{3}$, o que implica $r\left(e_{1}\right)<0$ e $r\left(e_{2}\right)=r\left(e_{2}\right)=0$. Em ambos os casos, a curvatura escalar satisfaz:

$$
\rho=-\frac{1}{2}\left(\lambda_{2}-\lambda_{3}\right)^{2}
$$

Já em $S l(2, \mathbb{R})$, encontramos $\lambda_{1}=2, \lambda_{2}=1$ e $\lambda_{3}=-1$. Substituindo nas fórmulas encontradas temos $r\left(e_{1}\right)=0$, do que é imediato pelo Lema 7 da Seção 5 que $r\left(e_{2}\right)<0$ e $r\left(e_{3}\right)=0$. Logo existe uma métrica invariante à esquerda em $S l(2, \mathbb{R})$ tal que $\operatorname{sgn}\left(r\left(e_{1}\right), r\left(e_{2}\right), r\left(e_{3}\right)\right)=(0,0,-)$ e $\rho<0$. Poderíamos alterar um pouco a métrica de maneira que, por exemplo, $\lambda_{1}=\frac{3}{2}, \lambda_{2}=-1$ e $\lambda_{3}=1$. Não é difícil verificar que nesse caso teremos $\operatorname{sgn}\left(r\left(e_{1}\right), r\left(e_{2}\right), r\left(e_{3}\right)\right)=(-,+,-)$. Mas, para qualquer métrica invariante à esquerda em $S l(2, \mathbb{R})$ temos curvatura escalar estritamente negativa. De fato, suponhamos que encontramos $\lambda_{1}, \lambda_{2}, \lambda_{3}$ quaisquer com, por exemplo, $\lambda_{1}<0<\lambda_{2}, \lambda_{3}$. Vimos que a curvatura escalar pode ser escrita em função de $\lambda_{i}$, como $\rho\left(\lambda_{1}, \lambda_{2}, \lambda_{3}\right)=\left(\lambda_{2} \lambda_{3}+\lambda_{1} \lambda_{3}+\lambda_{2} \lambda_{1}\right)-\frac{1}{2}\left(\lambda_{1}^{2}+\lambda_{2}^{2}+\lambda_{3}^{2}\right)$. Novamente fixemos $\lambda_{2} \mathrm{e}$ $\lambda_{3}$ e consideremos $\rho$ como função de $\lambda_{1}$. Temos que $\frac{\partial \rho}{\partial \lambda_{1}}=-\lambda_{1}+\lambda_{2}+\lambda_{3}$, o que mostra que para $\lambda_{1} \leq 0, \rho$ é uma função monótona crescente (vista como função de $\lambda_{1}$ ). Portanto $\rho\left(\lambda_{1}, \lambda_{2}, \lambda_{3}\right)<\rho\left(0, \lambda_{2}, \lambda_{3}\right)=-\frac{1}{2}\left(\lambda_{2}-\lambda_{3}\right)^{2} \leq 0$. Analogamente mostramos para os outros casos.

Concluímos com todas essas observações que se $G$ é $S l(2, \mathbb{R})$ ou $E(1,1)$, então dependendo da escolha de métrica invariante em $G$ que é feita, podemos ter $\operatorname{sgn}\left(r\left(e_{1}\right), r\left(e_{2}\right), r\left(e_{3}\right)\right)=$ $(+,-,-)$ ou $\operatorname{sgn}\left(r\left(e_{1}\right), r\left(e_{2}\right), r\left(e_{3}\right)\right)=(0,0,-)$. Entretanto, para qualquer métrica invariante à esquerda tomada em $G$, a curvatura escalar é estritamente negativa. 
Em 5.4 estudamos o grupo euclidiano $E(2)$. Vimos que sua álgebra não é abeliana, portanto $E(2)$ não é um grupo de Lie comutativo. De fato, encontramos $\lambda_{1}=0, \lambda_{2}=1$ e $\lambda_{3}=1$. Logo $r\left(e_{1}\right)=0, r\left(e_{2}\right)=0$ e $r\left(e_{3}\right)=0$. Portanto existe uma métrica invariante à esquerda em $E(2)$ com todas as curvaturas de Ricci iguais a zero. Como este grupo possui dimensão três e nesse caso as curvaturas de Ricci determinam a curvatura seccional, e esta determina a curvatura $R$, segue que $E(2)$ admite uma métrica invariante à esquerda flat (veja Teorema 9 da Seção 9). Podemos alterar a métrica de maneira que $\lambda_{1}=0$, e $\lambda_{3}>\lambda_{2}>0$. Novamente utilizando o Lema 7 da Seção 5 concluimos que nesse caso teríamos $\operatorname{sgn}\left(r\left(e_{1}\right), r\left(e_{2}\right), r\left(e_{3}\right)\right)=(-,+,-)$. Mostramos em 5.4 que $E(2)$ é solúvel. Logo, pelo Teorema 4 da Seção 4 , toda métrica invariante à esquerda em $E(2)$ ou é flat ou possui curvatura escalar estritamente negativa, ou seja, toda métrica que não é flat em $E(2)$ é tal que $\rho<0$. Mostramos que o grupo euclidiano não é comutativo, mas admite uma métrica invariante à esquerda flat. Toda métrica invariante à esquerda em $E(2)$ que não é flat é tal que $\operatorname{sgn}\left(r\left(e_{1}\right), r\left(e_{2}\right), r\left(e_{3}\right)\right)=(-,+,-)$ e possui curvatura escalar estritamente negativa.

Na próxima seção estudaremos o caso não unimodular. 


\section{O caso não-unimodular}

O seguinte lema descreve a álgebra de Lie de um grupo de Lie não-unimodular de dimensão três.

Lema 8. Seja $G$ um grupo de Lie não-unimodular de dimensão três. Então sua álgebra $\mathcal{G}$ possui uma base $\left\{e_{1}, e_{2}, e_{3}\right\}$ tal que:

$$
\begin{gathered}
{\left[e_{1}, e_{2}\right]=\alpha e_{2}+\beta e_{3}} \\
{\left[e_{1}, e_{3}\right]=\gamma e_{2}+\delta e_{3}} \\
{\left[e_{2}, e_{3}\right]=0}
\end{gathered}
$$

e a matriz $A=\left(\begin{array}{cc}\alpha & \beta \\ \gamma & \delta\end{array}\right)$ possui traço $\alpha+\delta=2$. Excluindo-se o caso em que $A=I$, temos que $\operatorname{det} A$ determina completamente a álgebra $\mathcal{G}$.

Demonstração: A álgebra de Lie $\mathcal{G}$ não é unimodular. Consideremos $\mathcal{U}$ como sendo o kernel unimodular de $\mathcal{G}$, definido na Seção 3 , que é o kernel do homomorfismo $\phi: \mathcal{G} \rightarrow \mathbb{R}$, definido por $\phi(x)=\operatorname{tr}\left(a d_{x}\right)$. Como mostramos ao final da seção $3, \mathcal{U}$ é um ideal unimodular de dimensão 2. Vamos mostrar que $\mathcal{U}$ é também comutativo. Seja $\left\{e_{2}, e_{3}\right\}$ uma base qualquer de $\mathcal{U}$ e sejam $x$, $y \in \mathcal{U}$, quaisquer. Então $[x, y]=\left[a_{2} e_{2}+a_{3} e_{3}, b_{2} e_{2}+b_{3} e_{3}\right]=a_{2} b_{3}\left[e_{2}, e_{3}\right]+a_{3} b_{2}\left[e_{3}, e_{2}\right]$, onde $a_{i}, b_{i}$ são reais quaisquer para $i=1,2$. Se verificarmos que $\left[e_{2}, e_{3}\right]=0$, então teremos $[x, y]=0$, para. quaisquer $x, y$. De fato, vamos escrever as matrizes de $a d_{e_{2}}$ e $a d_{e_{3}}$. Temos que:

$$
\begin{gathered}
a d_{e_{2}}\left(e_{2}\right)=0 \\
a d_{e_{2}}\left(e_{3}\right)=\left[e_{2}, e_{3}\right]=a e_{2}+b e_{3} \\
a d_{e_{3}}\left(e_{2}\right)=\left[e_{3}, e_{2}\right]=-\left[e_{2}, e_{3}\right]=-a e_{2}-b e_{3} \\
a d_{e_{3}}\left(e_{3}\right)=0 .
\end{gathered}
$$

Então $\left[a d_{e_{2}}\right]=\left(\begin{array}{ll}0 & a \\ 0 & b\end{array}\right)$ e $\left[a d_{e_{3}}\right]=\left(\begin{array}{cc}-a & 0 \\ -b & 0\end{array}\right)$. Mas temos que $e_{2}$ e $e_{3}$ pertencem à $\mathcal{U}$. Portanto $\operatorname{tr}\left(a d_{e_{2}}\right)=\operatorname{tr}\left(a d_{e_{3}}\right)=0$ e assim $a=0$ e $b=0$. Temos então $\left[e_{2}, e_{3}\right]=0$ e segue que $\mathcal{U}$ é comutativo.

Podemos escolher um $e_{1} \in \mathcal{G}$ ortogonal a. $\mathcal{U}$ tal que $\operatorname{tr}\left(a d_{e_{1}}\right)=2$. De fato, se escolhermos $\tilde{e}_{1} \in \mathcal{G}$ tal que $\operatorname{tr}\left(a d_{\tilde{e}_{1}}\right)$ não é igual a. 2 , digamos, $\operatorname{tr}\left(a d_{\tilde{e}_{1}}\right)=a+d \neq 0$, tomamos $e_{1}=\frac{2}{a+d} \tilde{e}_{1} \mathrm{e}$ teremos que $\operatorname{tr}\left(a d_{e_{1}}\right)=2$. Vamos escolher tal $e_{1}$. Considere a transformação $L: \mathcal{U} \rightarrow \mathcal{U}$ dada por $a d_{e_{1}}$ restrita à $\mathcal{U}$, isto é, $L(u)=\left[e_{1}, u\right]$, para $u \in \mathcal{U}$. Observe que se tomarmos $e_{1}$ e $e_{1}^{\prime}$ como 
anteriormente, segue que $\operatorname{tr}\left(a d_{\left(e_{1}-e_{1}^{\prime}\right)}\right)=\operatorname{tr}\left(a d_{e_{1}}\right)-\operatorname{tr}\left(a d_{e_{1}^{\prime}}\right)=0$ e portanto $e_{1}-e_{1}^{\prime}$ pertence a. $\mathcal{U}$ que é comutativo, e assim temos que $L$ é independente da escolha de $e_{1}$ e pela escolha que fizemos, seu traço é igual a. 2. Se $L(u)=\lambda u$, onde $\lambda$ é uma constante, então $\operatorname{tr}(L)=2 \lambda$. Ao mesmo tempo, $\operatorname{tr}(L)=2$ e portanto $\lambda=1$ e $L(u)=u$. Assim, $\left[e_{1}, e_{2}\right]=e_{2}$ e $\left[e_{1}, e_{3}\right]=e_{3}$ o que implica que $[x, y]$ é combinação linear de $x$ e $y$ para $x, y$ quaisquer. Estudamos esse caso no Exemplo 2.1, vamos então assumir que esse não é o caso. Vamos escolher $e_{2} \in \mathcal{U}$ tal que $e_{2}$ e $L\left(e_{2}\right)=e_{3}$ são linearmente independentes. Dessa maneira:

$$
\begin{gathered}
{\left[e_{1}, e_{2}\right]=L\left(e_{2}\right)=e_{3}} \\
{\left[e_{1}, e_{3}\right]=a d_{e_{1}}\left(e_{3}\right)=a e_{2}+b e_{3} .}
\end{gathered}
$$

Então, a matriz $\left[a d_{e_{1}}\right]$ é dada por $\left(\begin{array}{cc}0 & a \\ 1 & b\end{array}\right)$. Como o traço dessa neatriz é igual a 2 , temos que $b=2$. Além disso, $\operatorname{det} L=\operatorname{det}\left(a d_{e_{1}}\right)=-a$, portanto $a=-\operatorname{det} L=-D$. Podemos escrever:

$$
\begin{gathered}
{\left[e_{1}, e_{2}\right]=e_{3},} \\
{\left[e_{1}, e_{3}\right]=-D e_{2}+2 e_{3},} \\
{\left[e_{2}, e_{3}\right]=0}
\end{gathered}
$$

e assim mostramos que existe uma base $\left\{e_{1}, e_{2}, e_{3}\right\}$ de $\mathcal{G}$ tal que o colchete em $\mathcal{G}$ satisfaz as igualdades acima. Portanto o colchete, com relação a essa base especialmente escolhida, fica totalmente determinado, conhecendo-se o determinante da matriz $A$.

Assim como foi feito para o caso unimodular, queremos dar uma descrição da curvatura de Ricci. Seja $\mathcal{G}$ uma álgebra de Lie não-unimodular com uma dada uma métrica. Vamos escolher $e_{1} \in \mathcal{G}$ tal que $e_{1}$ é ortogonal ao kernel unimodular $\mathcal{U}$. Completemos $e_{1}$ a uma base ortonormal $B=\left\{e_{1}, e_{2}, e_{3}\right\}$ de $\mathcal{G}$, onde $B^{\prime}=\left\{e_{2}, e_{3}\right\}$ é uma base de $\mathcal{U}$. Temos o operador $L: \mathcal{U} \rightarrow \mathcal{U}$ dado por $L(u)=\left[e_{1}, u\right]$. Sejam $L\left(e_{2}\right)=\alpha^{\prime} e_{2}+\beta^{\prime} e_{3}$ e $L\left(e_{3}\right)=\gamma^{\prime} e_{2}+\delta^{\prime} e_{3}$ onde $\alpha^{\prime}, \beta^{\prime}, \gamma^{\prime}, \delta^{\prime} \in \mathbb{R}$. Seja $C^{\prime}=\left\{\tilde{e}_{2}, \tilde{e}_{3}\right\}$ tal que:

$$
\begin{gathered}
\tilde{e}_{2}=\cos \theta e_{2}+\sin \theta e_{3}, \\
\tilde{e}_{3}=-\sin \theta e_{2}+\cos \theta e_{3} .
\end{gathered}
$$

O conjunto $C^{\prime}=\left\{\tilde{e}_{2}, \tilde{e}_{3}\right\}$ é uma base ortonormal de $\mathcal{U}$. Para achar a matriz de $L$ nessa base, precisamos fazer $M^{t}[L]_{B^{\prime}} M$ onde $M$ é a matriz mudança de base de $B^{\prime}$ para $C^{\prime}$. Então temos:

$$
[L]_{C^{\prime}}=\left(\begin{array}{cc}
\cos \theta & \sin \theta \\
-\sin \theta & \cos \theta
\end{array}\right)\left(\begin{array}{cc}
\alpha^{\prime} & \gamma^{\prime} \\
\beta^{\prime} & \delta^{\prime}
\end{array}\right)\left(\begin{array}{cc}
\cos \theta & -\sin \theta \\
\sin \theta & \cos \theta
\end{array}\right)
$$




$$
=\left(\begin{array}{ll}
A(\theta) & C(\theta) \\
B(\theta) & D(\theta)
\end{array}\right),
$$

onde

$$
\begin{gathered}
A(\theta)=\alpha^{\prime} \cos ^{2} \theta+\delta^{\prime} \sin ^{2} \theta-\left(\beta^{\prime}+\gamma^{\prime}\right) \sin \theta \cos \theta, \\
C(\theta)=\gamma^{\prime} \cos ^{2} \theta-\beta^{\prime} \sin ^{2} \theta+\left(\alpha^{\prime}-\delta^{\prime}\right) \sin \theta \cos \theta \\
B(\theta)=-\gamma^{\prime} \sin ^{2} \theta+\beta^{\prime} \cos ^{2} \theta+\left(\alpha^{\prime}-\delta^{\prime}\right) \sin \theta \cos \theta \\
D(\theta)=\alpha^{\prime} \sin ^{2} \theta+\delta^{\prime} \cos ^{2} \theta+\left(\beta^{\prime}+\gamma^{\prime}\right) \sin \theta \cos \theta .
\end{gathered}
$$

Observe que $\left\langle L\left(\tilde{e}_{2}\right), L\left(\tilde{e}_{3}\right)\right\rangle=A(\theta) C(\theta)+B(\theta) D(\theta)$. Temos claramente que:

$$
\begin{gathered}
A(0) C(0)+B(0) D(0)=\alpha^{\prime} \gamma^{\prime}+\beta^{\prime} \delta^{\prime}, \\
A\left(\frac{\pi}{2}\right) C\left(\frac{\pi}{2}\right)+B\left(\frac{\pi}{2}\right) D\left(\frac{\pi}{2}\right)=-\delta^{\prime} \beta^{\prime}-\alpha^{\prime} \gamma^{\prime} .
\end{gathered}
$$

Como esta é uma função contínua em $\theta$, existe um $\theta^{\prime}, 0<\theta^{\prime}<\frac{\pi}{2}$ tal que:

$$
<L\left(e_{2}^{\prime}\right), L\left(e_{3}^{\prime}\right)>=A\left(\theta^{\prime}\right) C\left(\theta^{\prime}\right)+B\left(\theta^{\prime}\right) D\left(\theta^{\prime}\right)=0
$$

onde $e_{2}^{\prime}=e_{2} \cos \theta^{\prime}+e_{3} \sin \theta^{\prime}$ e $e_{3}^{\prime}=-e_{2} \sin \theta^{\prime}+e_{3} \cos \theta^{\prime}$. Chamemos $\alpha=A\left(\theta^{\prime}\right), \beta=B\left(\theta^{\prime}\right)$, $\gamma=C\left(\theta^{\prime}\right)$ e $\delta=D\left(\theta^{\prime}\right)$. Então mostramos que existe uma base $\left\{e_{1}, e_{2}^{\prime}, e_{3}^{\prime}\right\}$ de $\mathcal{G}$ tal que $e_{1}$ é ortogonal a $\mathcal{U}$ e as imagens $L\left(e_{2}^{\prime}\right)$ e $L\left(e_{3}^{\prime}\right)$ são ortogonais, além disso não é difícil ver que $\alpha+\delta=A(\theta)+D(\theta)$, para qualquer $\theta$, em particular $\alpha+\delta=\alpha^{\prime}+\delta^{\prime} \neq 0$, e claramente $\alpha \gamma+\beta \delta=0$. O colchete satisfaz:

$$
\begin{gathered}
{\left[e_{1}, e_{2}^{\prime}\right]=\alpha e_{2}^{\prime}+\beta e_{3}^{\prime},} \\
{\left[e_{1}, e_{3}^{\prime}\right]=\gamma e_{2}^{\prime}+\delta e_{3}^{\prime},} \\
{\left[e_{2}^{\prime}, e_{3}^{\prime}\right]=0 .}
\end{gathered}
$$

O seguinte lema relaciona tais constantes com a curvatura de Ricci.

Lema 9. Seja $\left\{e_{1}, e_{2}, e_{3}\right\}$ uma base escolhida como anteriormente. Tal base diagonaliza a forma quadrática de Ricci, e as curvaturas principais são dadas por:

$$
\begin{gathered}
r\left(e_{1}\right)=-\alpha^{2}-\delta^{2}-\frac{1}{2}(\beta+\gamma)^{2}, \\
r\left(e_{2}\right)=-\alpha(\alpha+\delta)+\frac{1}{2}\left(\gamma^{2}-\beta^{2}\right), \\
r\left(e_{3}\right)=-\delta(\alpha+\delta)+\frac{1}{2}\left(\beta^{2}-\gamma^{2}\right) .
\end{gathered}
$$


Demonstração: Vamos calcular as constantes estruturais da álgebra $\mathcal{G}$ com relação à esta base escolhida. Por exemplo, $\alpha_{23 k}=\alpha_{32 k}=0$ para $k=1,2,3$, pois $\mathcal{U}$ é comutativo. Temos também $\alpha_{i j 1}=0$ para todo $i, j$, pois $e_{1}$ foi escolhido ortogonal à $\mathcal{U}$. Finalizando, temos:

$$
\begin{aligned}
& \alpha_{122}=<\left[e_{1}, e_{2}\right], e_{2}>=\alpha, \\
& \alpha_{123}=<\left[e_{1}, e_{2}\right], e_{3}>=\beta, \\
& \alpha_{132}=<\left[e_{1}, e_{3}\right], e_{2}>=\gamma, \\
& \alpha_{133}=<\left[e_{1}, e_{3}\right], e_{3}>=\delta .
\end{aligned}
$$

Podemos calcular agora o tensor de Riemann. Por exemplo,

$$
\begin{gathered}
R_{e_{2} e_{1}} e_{2}=\nabla_{\left[e_{2}, e_{1}\right]} e_{2}-\nabla_{e_{2}} \nabla_{e_{1}} e_{2}+\nabla_{e_{1}} \nabla_{e_{2}} e_{2}= \\
-\alpha \nabla_{e_{2}} e_{2}-\beta \nabla_{e_{3}} e_{2}-\nabla_{e_{2}} \nabla_{e_{1}} e_{2}+\nabla_{e_{1}} \nabla_{e_{2}} e_{2}
\end{gathered}
$$

Com as constantes estruturais encontradas, não é difícil calcular que $\nabla_{e_{2}} e_{2}=\alpha e_{1}$ e $\nabla_{e_{1}} e_{1}=0$, usando a fórmula $\nabla_{e_{i}} e_{j}=\sum_{k} \frac{1}{2}\left(\alpha_{i j k}-\alpha_{j k i}+\alpha_{k i j}\right) e_{k}$. Analogamente:

$$
\begin{aligned}
& \nabla_{e_{1}} e_{2}=\frac{1}{2}(\beta-\gamma) e_{3}, \\
& \nabla_{e_{2}} e_{3}=\frac{1}{2}(\gamma+\beta) e_{1} \\
& \nabla_{e_{1}} e_{3}=\frac{1}{2}(\gamma-\beta) e_{2}
\end{aligned}
$$

e, pela simetria da conexão de Riemann, temos $\nabla_{e_{i}} e_{j}=\nabla_{e_{j}} e_{i}$. Assim não é difícil encontrar:

$$
\begin{aligned}
& R_{e_{2} e_{1}} e_{2}=-\alpha^{2} e_{1}-\frac{\beta}{2}(\beta+\gamma) e_{1}-\frac{1}{4}\left(\beta^{2}-\gamma^{2}\right)^{2} e_{1} \\
& R_{e_{3} e_{1}} e_{3}=-\frac{\gamma}{2}(\gamma+\beta) e_{1}-\delta^{2} e_{1}-\frac{1}{4}\left(\gamma^{2}-\beta^{2}\right) e_{1}
\end{aligned}
$$

Então, por exemplo,

$$
\hat{r}\left(e_{1}\right)=R_{e_{2} e_{1}} e_{2}+R_{e_{3} e_{1}} e_{3}=\left(-\alpha^{2}-\delta^{2}-\frac{1}{2}(\beta+\gamma)^{2}\right) e_{1} .
$$

Analogamente podemos achar

$$
\begin{aligned}
& \hat{r}\left(e_{2}\right)=\left(-\alpha(\alpha+\delta)+\frac{1}{2}\left(\gamma^{2}+\beta^{2}\right)\right) e_{2} \\
& \hat{r}\left(e_{3}\right)=\left(-\delta(\alpha+\delta)+\frac{1}{2}\left(\beta^{2}-\gamma^{2}\right)\right) e_{3}
\end{aligned}
$$


Logo $\left\{e_{1}, e_{2}, e_{3}\right\}$ é uma base de autovetores de $\hat{r}, \operatorname{com} r\left(e_{i}\right)$ igual a cada autovalor correspondente a. $e_{i}$.

Seja $\left\{e_{1}, e_{2}, e_{3}\right\}$ uma base de $\mathcal{G}$ escolhida como no Lema 9. Podemos relacionar o determinante $D$ da matriz $\left(\begin{array}{cc}\alpha & \gamma \\ \beta & \delta\end{array}\right)$ com as curvaturas de Ricci encontradas no Lema 9. Este é o resultado do próximo teorema:

Teorema 6. Se o determinante $D$ é estritamente negativo, então toda métrica invariante à esquerda em $G$ é tal que sgn $\left(r\left(e_{1}\right), r\left(e_{2}\right), r\left(e_{3}\right)\right)=(-,-,+)$. Se $D=0$, então toda métrica invariante à esquerda em $G$ é tal que ou sgn $\left(r\left(e_{1}\right), r\left(e_{2}\right), r\left(e_{3}\right)\right)=(-,-,+)$ ou $\operatorname{sgn}\left(r\left(e_{1}\right), r\left(e_{2}\right), r\left(e_{3}\right)\right)=$ $(0,-,-)$. Se $D>0$, então $\operatorname{sgn}\left(r\left(e_{1}\right), r\left(e_{2}\right), r\left(e_{3}\right)\right)=(-,-,+)$ e para $D>1$ existe uma métrica invariante à esquerda de curvatura de Ricci constante negativa. Em todos os casos, a curvatura escalar é estritamente negativa.

Demonstração: Façamos uma mudança de variáveis de maneira que $\alpha+\delta=2$. Como escolhemos $\left\{e_{1}, e_{2}, e_{3}\right\}$ tais que $L\left(e_{2}\right)$ e $L\left(e_{3}\right)$ são ortogonais, podemos escrever:

$$
\begin{gathered}
\alpha=1+\xi \\
\beta=(1+\xi) \eta \\
\gamma=-(1-\xi) \eta \\
\delta=1-\xi
\end{gathered}
$$

onde podemos supor que $\xi \geq 0$ e $\eta \geq 0$. Então $\alpha^{2}=(1+\xi)^{2}, \delta^{2}=(1-\xi)^{2}, \beta+\gamma=2 \eta \xi$, $\gamma^{2}=\eta^{2}(1-\xi)^{2}$ e $\beta^{2}=\eta^{2}(1+\xi)^{2}$. Portanto, pelo Lema 9 desta seção:

$$
\begin{gathered}
r\left(e_{1}\right)=-(1+\xi)^{2}-(1-\xi)^{2}-\frac{1}{2}(2 \eta \xi)^{2}=-2\left(1+\xi^{2}\left(1+\eta^{2}\right)\right) \leq-2 \\
r\left(e_{2}\right)=-2\left(1+\xi\left(1+\eta^{2}\right)\right) \\
r\left(e_{3}\right)=-2\left(1-\xi\left(1+\eta^{2}\right)\right) .
\end{gathered}
$$

Se $\xi=0$ e $\eta=0$, então temos $\alpha=1, \beta=0, \gamma=0$ e $\delta=1$ e a matriz $A$ é igual à identidade. Mas estamos excluindo esse caso. Então sejam $\xi>0$ ou $\eta>0$. O determinante da matriz $A$ nesse caso é dado por $D=\left(1-\xi^{2}\right)\left(1+\eta^{2}\right)$; suponhamos que $D<0$. Então devemos ter que $\xi>1$ e $\eta \geq 0$. Assim,

$$
r\left(e_{3}\right)>-2\left(1-\left(1+\eta^{2}\right)\right)=2 \eta^{2} \geq 0
$$


e $r\left(e_{2}\right) \leq-2$. Logo $\operatorname{sgn}\left(r\left(e_{1}\right), r\left(e_{2}\right), r\left(e_{3}\right)\right)=(-,-,+)$. Se $D=0$ então temos $\xi=1(\xi \geq 0)$ e $\eta \geq 0$. Se $\xi=1$ e $\eta>0$, então

$$
r\left(e_{3}\right)=-2\left(1-1\left(1+\eta^{2}\right)\right)=-2\left(-\eta^{2}\right)=2 \eta^{2} \geq 0 .
$$

Portanto nesse caso, $\operatorname{sgn}\left(r\left(e_{1}\right), r\left(e_{2}\right), r\left(e_{3}\right)\right)=(-,-,+)$. Se $\xi=1$ e $\eta=0$, então é imediato verificar que $r\left(e_{1}\right)=-4, r\left(e_{2}\right)=-4$ e $r\left(e_{3}\right)=0$.

Suponhamos que $D>0$. Então devemos ter $0 \leq \xi<1$. Se $\xi=0$, como $\eta>0$, teremos $D>1$ e não é difícil ver que $r\left(e_{1}\right)=r\left(e_{2}\right)=r\left(e_{3}\right)=-2$, ou seja, existe uma métrica invariante à esquerda de curvatura de Ricci constante e negativa. Se $0<\xi<1$ e $\eta=0$, não é difícil concluir que $\operatorname{sgn}\left(r\left(e_{1}\right), r\left(e_{2}\right), r\left(e_{3}\right)\right)=(-,-,-)$. Por outro lado, se $0<\xi<1$ e $\eta>0$, obtemos $\operatorname{sgn}\left(r\left(e_{1}\right), r\left(e_{2}\right), r\left(e_{3}\right)\right)=(-,-,+)$. Em geral, a curvatura escalar é dada por:

$$
r\left(e_{1}\right)+r\left(e_{2}\right)+r\left(e_{3}\right)=-6-2 \xi^{2}\left(1+\eta^{2}\right)-4 \xi \eta^{2}<0
$$

$\mathrm{e}$ isto conclui a demonstração.

Portanto, no caso de grupos de Lie não-unimodulares, pelo menos duas curvaturas principais de Ricci são estritamente negativas. Comparando com o caso unimodular estudado ao final da Seção 6, percebemos que nenhum grupo de Lie de dimensão três admite uma métrica invariante à esquerda tal que os sinais das curvaturas principais de Ricci satisfazem $(+,+,-)$ ou $(+, \pm, 0)$. 


\section{Métricas Bi-invariantes}

Nesta seção consideraremos um grupo de Lie munido de uma métrica bi-invariante, ou seja, uma métrica invariante à esquerda e à direita. Mostramos na Proposição 1 da Seção 2 que um grupo de Lie possuir uma métrica bi-invariante é equivalente a existir um produto interno em $T_{e} G \simeq \mathcal{G} A d$-invariante, isto é, tal que $\left\langle A d_{g}(u), A d_{g}(v)\right\rangle=\langle u, v>$, para todos $u, v \in \mathcal{G}$, $g \in G$. Vimos que isto implica em a transformação adjunta ser anti-adjunta, para todo elemento da álgebra e vale a recíproca se o grupo é conexo. É conveniente definirmos que uma métrica em $\mathcal{G}$ é bi-invariante se $a d_{x}$ é anti-adjunta, para todo $x \in \mathcal{G}$.

Calculamos também a curvatura seccional no caso de métrica bi-invariante, utilizando a fórmula de Koszul para mostrar que a conexão satisfaz $\nabla_{x}=\frac{1}{2} a d_{x}$ e $R(x, y)=\frac{1}{4} a d_{[x, y]}$. Finalmente encontramos:

$$
k(x, y)=\frac{1}{4}<[x, y],[x, y]>.
$$

Segue que $k(x, y) \geq 0$ e vale a igualdade se e somente se $[x, y]=0$. Lembremos que a curvatura de Ricci na direção de um elemento unitário $x$ da álgebra é soma de curvaturas seccionais de planos gerados por $x$ e elementos de uma base ortonormal de $\mathcal{G}$, melhor dizendo, $r(x)=\sum_{i} k\left(x, e_{i}\right)$. Neste caso, como $k\left(x, e_{i}\right) \geq 0$, para todo $i$, então $r(x) \geq 0$ e vale a igualdade se e somente se $k\left(x, e_{i}\right)=0$, para todo $i$, ou seja, se e somente se $\left[x, e_{i}\right]=0$, o que é equivalente dizer que $x$ pertence a centro da álgebra. A curvatura de Ricci também pode ser caracterizada como o traço da aplicação $y \mapsto R(x, y)(x)$. Com métrica bi-invariante, esta aplicação é dada por:

$$
y \mapsto \frac{1}{4} a d_{[x, y]}(x)=-\frac{1}{4} a d_{x}^{2}(y) .
$$

A forma bilinear em $\mathcal{G} \times \mathcal{G}$, dada por $\beta(x, y)=\operatorname{tr}\left(a d_{x} a d_{y}\right)$ é claramente simétrica e é chamada forma de Killing em $\mathcal{G}$. Note pelo que escrevemos acima que em um grupo de Lie com métrica bi-invariante, temos $r(x)=-\frac{1}{4} \beta(x, x)$, e isto mostra que $r(x)$ é independente de uma escolha particular de métrica bi-invariante.

O principal resultado desta seção é a classificação dos grupos de Lie conexos que admitem métrica bi-invariante. Antes de tal resultado, mostremos que todo grupo de Lie compacto admite uma métrica bi-invariante. Porém, para mostrarmos este resultado precisamos de alguns resultados e definições sobre variedades Riemannianas e para tanto recomendamos ao leitor o Apêndice A.

Lema 10. Um grupo de Lie $G$ compacto e conexo admite uma métrica bi-invariante.

Demonstração: Vamos usar a Proposição 1 da Seção 2 e alguns fatos contidos no Apêndice A. Seja $\langle\cdot, \cdot\rangle_{e}$ um produto interno en $T_{e} G$. O grupo $G$ admite um único elemento de volume 
$\Omega$ bi-invariante tal que $\operatorname{vol}(G)=1$ (veja Apêndice A). Dados $X, Y \in T_{e} G$, vamos definir uma função em $G$ por:

$$
f(g)=<\operatorname{Ad}(g) X, A d(g) Y>_{e} .
$$

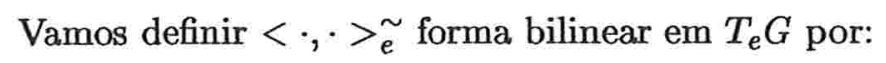

$$
<X, Y>_{\tilde{e}}^{\sim}=\int_{G} f(g) \Omega=\int_{G}<\operatorname{Ad}(g) X, A d(g) Y>_{e} \Omega .
$$

Pelas propriedades de integral e por $\langle\cdot, \cdot\rangle_{e}$ ser um produto interno em $T_{e} G$, segue que $<\cdot, \cdot>_{\tilde{e}}^{\sim}$ é, de fato, um produto interno em $T_{e} G$. Pela Proposição 1 da Seção 2, $G$ admitir métrica biinvariante é equivalente a existir um produto interno Ad-invariante em $T_{e} G$. Vamos mostrar que $\langle\cdot, \cdot\rangle_{e}^{\sim}$ é este tal produto interno. Ou seja, precisamos mostrar que:

$$
<\operatorname{Ad}(a)(X), \operatorname{Ad}(a)(Y)>_{e}^{\tilde{e}}=<X, Y>_{\tilde{e}}^{\tilde{e}}
$$

para todo $a \in G$. De fato,

$$
\begin{gathered}
<\operatorname{Ad}(a)(X), \operatorname{Ad}(a)(Y)>_{e}^{\sim} \\
=\int_{G}<\operatorname{Ad}(g)(\operatorname{Ad}(a) X), \operatorname{Ad}(g)(\operatorname{Ad}(a) Y)>_{e} \Omega \\
=\int_{G}<\operatorname{Ad}(g a)(X), \operatorname{Ad}(g a)(Y)>_{e} \Omega \\
=\int_{G} f\left(R_{a}(g)\right) \Omega .
\end{gathered}
$$

A segunda igualdade ocorre pois $A d: G \rightarrow A u t(\mathcal{G})$ é um homomorfismo. Observe que $R_{a}: G \rightarrow$ $G$ é um difeomorfismo, então pelas propriedades de integral de uma $n$-forma em $G$ vistas no Apêndice A, pelo fato de $R_{a}$ preservar a orientação de $G$ :

$$
\int_{G} R_{a}^{*} f(g) \Omega=\int_{G} f(g) \Omega
$$

e a primeira integral é exatamente $\int_{G} f\left(R_{a}(g)\right) \Omega$, e assim segue o resultado, isto é, $\langle\cdot, \cdot\rangle_{e}^{\sim}$ é um produto interno em $T_{e} G A d$-invariante.

Com isso é possível classificarmos os grupos de Lie conexos munidos de métrica bi-invariante. Este é o próximo teorema.

Teorema 7. Um grupo de Lie conexo $G$ admite métrica bi-invariante se e somente se $G$ é isomorfo a $H \times \mathbb{R}^{n}$, onde $H$ é um grupo de Lie compacto. 
Demonstração: Em um primeiro momento, olhemos como é descrita a álgebra associada nesta situação. Se a métrica em $\mathcal{G}$ é bi-invariante, sabemos que a transformação $a d_{X}$ é antiadjunta para todo $X$. Seja $\mathcal{A}$ um ideal em $\mathcal{G}$ e $a$ um elemento pertencente ao complemento ortogonal de $\mathcal{A}$, que denotamos por $\mathcal{A}^{\perp}$. Seja $y \in \mathcal{G}$ qualquer. Então observemos que, para $x \in \mathcal{A}$ temos:

$$
<x,[y, a]>=-<[y, x], a>=0
$$

pois $[y, x] \in \mathcal{A}$. Logo $[y, a] \in \mathcal{A}^{\perp}$, para qualquer $y \in \mathcal{G}$. Portanto $\mathcal{A}^{\perp}$ é um ideal na álgebra e sabemos que $\mathcal{G}=\mathcal{A} \oplus \mathcal{A}^{\perp}$. Se estes ideais forem simples, então conseguimos escrever a álgebra como soma direta ortogonal de ideais simples. Se não, prosseguimos fazendo a mesma decomposição ortogonal. Ao final deste processo, obteremos uma decomposição da álgebra em soma direta ortogonal do tipo:

$$
\mathcal{G}=\mathcal{A}_{1} \oplus \mathcal{A}_{2} \oplus \cdots \oplus \mathcal{A}_{k}
$$

onde $\mathcal{A}_{i}$ ou é um ideal simples, ou é abeliano unidimensional. Definindo:

$$
\begin{aligned}
& \mathcal{A}=\sum_{1=\operatorname{dim} \mathcal{A}_{i}} \mathcal{A}_{i} \\
& \mathcal{B}=\sum_{1<\operatorname{dim} \mathcal{A}_{i}} \mathcal{A}_{i}
\end{aligned}
$$

temos $\mathcal{G}=\mathcal{A} \oplus \mathcal{B}$, onde $\mathcal{A}$ é um ideal abeliano. É uma consequência do teorema de Ado (veja [23]) que se $\mathcal{G}$ é uma álgebra de Lie qualquer, então existe um grupo de Lie conexo e simplesmente conexo cuja álgebra associada é $\mathcal{G}$. Portanto, cada componente $\mathcal{A}_{i}$ de $\mathcal{B}$ é álgebra de Lie de um grupo de Lie simplesmente conexo $A_{i}$, além disso observe que o centro de cada $\mathcal{A}_{i}$ é trivial, pois se não fosse teríamos um ideal não trivial, absurdo. Note que $\mathcal{G}$ induz uma métrica bi-invariante em qualquer subálgebra, portanto em particular a curvatura de Ricci em $\mathcal{A}_{i} \subset \mathcal{B}$ é não negativa e é nula se e somente se o elemento pertence ao centro. Como o centro é trivial, segue que todas as curvaturas de Ricci são estritamente positivas. Pelo Teorema 1 da Seção 3, segue que cada $A_{i}$ é compacto. Se $\mathcal{A}_{i}$ pertence à soma $\mathcal{A}$, sabemos que seu grupo de Lie conexo e simplesmente conexo correspondente $A_{i}$ é isomorfo a $\mathbb{R}$.

Seja $\tilde{G}$ o recobrimento universal de $G$. A álgebra de Lie do recobrimento é isomorfa à $\mathcal{G}=\mathcal{A} \oplus \mathcal{B}$. Como $\tilde{G}$ é simplesmente conexo, àquele isomorfismo de álgebras corresponde um isomorfismo de grupos

$$
\tilde{G} \simeq \mathbb{R} \times \mathbb{R} \times \cdots \mathbb{R} \times A_{1} \times \cdots A_{n}
$$

onde cada $A_{i}$ é compacto. Logo $\tilde{G} \simeq \mathbb{R}^{n} \times \mathbf{H}$, onde $\mathbf{H}$ é um grupo de Lie compacto. Seja $\pi: \tilde{G} \rightarrow G$ a aplicação de recobrimento. Sabemos que sua diferencial $d \pi: T_{e} \tilde{G} \rightarrow T_{e} G$ é um 
isomorfismo e, portanto, é um difeomorfismo local. Logo, o conjunto $\Pi=\operatorname{ker} \pi=\pi^{-1}(\{e\})$ é um subgrupo normal discreto de $\tilde{G}$, onde discreto significa que se $\sigma \in \Pi$, então existe um aberto $V$ de $\tilde{G}$ tal que $V \cap \Pi=\{\sigma\}$. Podemos assim considerar o quociente $\tilde{G} / \Pi$ e existe um isomorfismo de grupos de Lie $G \simeq \tilde{G} / \Pi$. Seja $P_{1}(\Pi)$ a projeção de $\Pi$ em $\mathbb{R}^{n}$. Como este conjunto é um subgrupo discreto de $\mathbb{R}^{n}$, existem $e_{1}, \cdots, e_{d}$ elementos de $P_{1}(\Pi)$ linearmente independentes sobre $\mathbb{R}$ tais que cada elemento de $P_{1}(\Pi)$ é escrito unicamente como combinação linear $n_{1} e_{1}+\cdots+n_{d} e_{d}$ onde $n_{i}$ são inteiros, $i=1, \cdots, d$. Ou seja:

$$
P_{1}(\Pi)=\left\{\sum_{i=1}^{d} n_{i} e_{i} ; n_{i} \in \mathbf{Z}, i=1, \cdots, d\right\} .
$$

Seja $V$ o subespaço de $\mathbb{R}^{n}$ gerado por $\left\{e_{1}, \cdots, e_{d}\right\}$. Então podemos escrever $\mathbb{R}^{n}=V \oplus V^{\perp} \mathrm{e}$ assim:

$$
\begin{aligned}
G \simeq \tilde{G} / \Pi \simeq & \left(\mathbb{R}^{n} \times \mathbf{H}\right) / \Pi \simeq\left(V^{\perp} \times V \times H\right) / \Pi \\
& \simeq V^{\perp} \times(V \times H) / \Pi
\end{aligned}
$$

pois $\Pi$ age trivialmente em $V^{\perp}$ e o segundo termo do produto é compacto. Reciprocamente suponhamos que o grupo é isomorfo a $\mathbb{R}^{n} \times H$, onde $H$ é um grupo de Lie compacto e conexo. Observe que $\mathbb{R}^{n}$ é um grupo de Lie abeliano, isto é, o colchete é identicamente nulo. Portanto a aplicação adjunta $a d_{x}$ é anti-adjunta (pois é identicamente nula) para todo $x \in \mathbb{R}^{n}$, logo qualquer forma bilinear positiva $\langle\cdot, \cdot\rangle_{e}$ definida na sua álgebra (que é o próprio $\mathbb{R}^{n}$ ) estende-se naturalmente a uma métrica bi-invariante no grupo. Pelo lema anterior, como $H$ é um grupo de Lie conexo e compacto, admite uma métrica bi-invariante. Considerando a métrica Riemanniana produto em $H \times \mathbb{R}^{n}$, obtemos o resultado.

Veremos agora que se além disso $G$ for simples, então tal métrica é única, a menos de multiplicação por um escalar real positivo. É o próximo lema:

Lema 11. Seja $G$ um grupo de Lie compacto cuja álgebra associada é simples. Então a métrica bi-invariante que existe em $G$ é única a menos de multiplicação por uma constante real positiva. Além disso tal métrica possui curvatura de Ricci constante e é dada pela forma de Killing.

Demonstração: Lembremos da álgebra linear que em um espaço vetorial $V$ de dimensão finita com produto interno $\langle\cdot, \cdot\rangle$, um operador linear $S$ em $V$ é chamado positivo se é autoadjunto e $\langle S(v), v\rangle$ é estritamente positivo, para todo $v \in V$. Sabemos que existe um isomorfismo entre o conjunto dos operadores lineares positivos de $V$ e o conjunto das formas bilineares simétricas definidas positivas. Tal isomorfismo leva um operador $S$ na forma bilinear 
$\langle S(u), v\rangle$. Seja agora $\langle\cdot, \cdot\rangle$ uma métrica bi-invariante em $G$, que sabemos existir pelo Lema 10 e consideremos a sua restrição à $\mathcal{G}$. Pelo resultado mencionado, qualquer outro produto interno em $\mathcal{G}$ é escrito como $\langle S(x), y\rangle_{e}$, onde $S$ é um operador positivo em $\mathcal{G}$, denotemos tal métrica por $\langle\cdot, \cdot\rangle^{\sim}$. A aplicação adjunta é anti-adjunta em ambas as métricas, portanto:

$$
\begin{gathered}
<\operatorname{ad}(u)(x), y>^{\sim}=-<x, \operatorname{ad}(u)(y)>^{\sim} \Rightarrow \\
<[u, x], y>^{\sim}=-<x,[u, y]>^{\sim} \Rightarrow \\
<S([u, x]), y>_{e}=-<S x,[u, y]>_{e}=<\operatorname{ad}(u)(S x), y>_{e} \Rightarrow \\
<S \circ \operatorname{ad}(u)(x), y>_{e}=<\operatorname{ad}(u) \circ(S x), y>_{e} \Rightarrow \\
<\left(S \circ \operatorname{ad}_{i}(u)\right)(x)-(\operatorname{ad}(u) \circ(S))(x), y>_{e}=0,
\end{gathered}
$$

para todo $y \in \mathcal{G}$. Segue assim que a aplicação adjunta comuta com $S$. Com isso, se $v$ pertence a um autoespaço de $S$ correspondente a um autovalor $\lambda$ (que sabemos ser real e estritamente positivo, pois $S$ é positivo), então $a d(u)(S(v))=[u, S v]=[u, \lambda v]=\lambda[u, v]$. Ao mesmo tempo, $\operatorname{ad}(u)(S(v))=S(\operatorname{ad}(u)(v))=S([u, v])$, o que mostra que a aplicação adjunta leva cada autoespaço de $S$ nele mesmo. Isto em particular implica que cada auto-espaço é um ideal de $\mathcal{G}$. Como a álgebra é simples, ela possui somente um autoespaço, isto é, $S(x)=\lambda x$, para todo $x \in \mathcal{G}$ e para algum $\lambda>0$ real. Portanto:

$$
\left.<x, y>^{\sim}=\lambda<x, y\right\rangle_{e}
$$

onde $\lambda>0$. Isto mostra a unicidade da métrica.

Considerando ainda a métrica bi-invariante $\langle\cdot, \cdot>\operatorname{em~} \mathcal{G}$, lembremos que a curvatura principal de Ricci satisfaz a relação $r(x)=<\hat{r}(x), x>$, onde $\hat{r}$ é a transformação auto-adjunta de Ricci. Vimos também que neste caso $r(x) \geq 0$ e vale a igualdade se e somente se $x$ pertence ao centro da álgebra. Como o centro de $\mathcal{G}$ é um ideal e a álgebra é simples, a igualdade não ocorre. Logo $r(x)>0$, para todo $x \in \mathcal{G}$. Segue que a transformação $\hat{r}$ é positiva e $\langle x, y\rangle^{\sim}=<\hat{r}(x), y>$ é um produto interno em $\mathcal{G}$. Este produto interno pode ser estendido naturalmente a uma métrica invariante à esquerda em $G$. Nesta situação esta métrica também é invariante à direita. Usando o mesmo argumento acima, segue que $\hat{r}(x)=\lambda x$ para todo $x \in \mathcal{G}$ e algum $\lambda$ constante real estritamente positvo. Se $u \in \mathcal{G}$ é unitário, temos $r(u)=\langle\lambda u, u\rangle=\lambda\rangle 0$. Para finalizar a demonstração, verifiquemos que $\langle x, y\rangle=-\frac{1}{4} \beta(x, y)$, onde $\beta$ é a forma de Killing, é um produto interno bi-invariante em $\mathcal{G}$. A forma de Killing $\beta: \mathcal{G} \times \mathcal{G} \rightarrow \mathbb{R}$ é dada por $\beta(x, y)=\operatorname{tr}(\operatorname{ad}(x) \operatorname{ad}(y))$. Esta forma bilinear é evidentemente simétrica, além disso em uma álgebra simples e compacta satisfaz $\beta(x, x) \leq 0$, se $x \in \mathcal{G} \neq 0$. Portanto $<x, y>=-\frac{1}{4} \beta(x, y)$ é 
um produto interno em $\mathcal{G}$. Se mostrarmos que este produto interno é bi-invariante, teremos de maneira natural pela homogeneidade uma métrica Riemanniana bi-invariante em todo o grupo. De fato, verfifiquemos que nesta métrica a aplicação adjunta é anti-adjunta para todo elemento da álgebra. Sejam $x, y, z \in \mathcal{G}$ quaisquer:

$$
\begin{gathered}
<\operatorname{ad}(x)(y), z>=<[x, y], z>=-\frac{1}{4} \operatorname{tr}(\operatorname{ad}([x, y]) \operatorname{ad}(z)) \\
=-\frac{1}{4} \operatorname{tr}([\operatorname{ad}(x), \operatorname{ad}(y)] \operatorname{ad}(z)) \\
=-\frac{1}{4} \operatorname{tr}((\operatorname{ad}(x) \operatorname{ad}(y)-\operatorname{ad}(y) \operatorname{ad}(x)) \operatorname{ad}(z)) \\
=-\frac{1}{4}(\operatorname{tr}(\operatorname{ad}(x) \operatorname{ad}(y) \operatorname{ad}(z))-\operatorname{tr}(\operatorname{ad}(y) \operatorname{ad}(x) \operatorname{ad}(z)) \\
=-\frac{1}{4} \operatorname{tr}(\operatorname{ad}(y) \operatorname{ad}(z) \operatorname{ad}(x)-\operatorname{ad}(y) \operatorname{ad}(x) \operatorname{ad}(z))=<y,[z, x]>
\end{gathered}
$$

Isto finaliza a demonstração.

Em uma variedade Riemanniana qualquer, podemos relacionar as conexões $\nabla$ e $\tilde{\nabla}$ com relação às métricas Riemannianas $\langle\cdot, \cdot\rangle$ e $\lambda\langle\cdot, \cdot\rangle$, onde $\lambda$ é uma constante estritamente positiva. Utilizando a fórmula de Koszul, podemos facilmente concluir que nesta situação as conexões coincidem, isto é, $\nabla=\tilde{\nabla}$ (geralmente mostra-se que se $(M,<\cdot, \cdot>$ ) é uma variedade Riemanniana qualquer e se $\langle\cdot, \cdot\rangle^{\sim}=f^{2}\langle\cdot, \cdot\rangle$, onde $f$ é uma função $C^{\infty}$ em $M$ que nunca se anula, então $\tilde{\nabla}_{X} Y-\nabla_{X} Y=\left(\frac{1}{f}\right)(X(f) Y+Y(f) X-\operatorname{grad}(f)<X, Y>$ ) é a relação entre as conexões). Voltando ao caso estudado, como as conexões coincidem, o mesmo ocorre com o tensor de curvatura. As curvaturas seccionais satisfazem $\tilde{k}=\lambda^{-1} k$ e a curvatura de Ricci não se altera, isto é, $r=\tilde{r}$. Então inicialmente podemos escolher uma métrica de maneira que $r(x) \equiv<x, x>$, ou seja, de maneira que a curvatura de Ricci seja constante igual a +1 .

Suponhamos agora que $G$ é um grupo de Lie conexo cujo recobrimento universal $\tilde{G}$ é compacto, de maneira que admite uma métrica bi-invariante. De acordo com a construção do Lema 11, sua álgebra de Lie se decompõe como soma direta ortogonal de ideais simples e assim existe um isomorfismo $\tilde{G} \simeq A_{1} \times \cdots \times A_{k}$, onde $A_{i}$ são grupos de Lie simples. Pelo Lema 12, cada um destes grupos é equipado com uma única métrica bi-invariante de curvatura de Ricci constante igual a +1 . Utilizando a métrica produto, $\tilde{G}$ admite uma métrica bi-invariante de curvatura de Ricci constante igual a +1 . Sabemos que $\pi$ é uma isometria local, de maneira que, localmente, $G$ admite uma métrica bi-invariante de curvatura de Ricci constante igual a +1 . 


\section{Grupos de Lie com curvatura zero}

Gostaríamos de classificar agora os grupos de Lie que admitem métrica invariante à esquerda. flat, isto é, tal que a curvatura é identicamente nula. De acordo com o Lema 1 da Seção 2 , se $G$ é abeliano então toda métrica invariante à esquerda em $G$ é flat. Porém vimos na Seção 6 que $E(2)$ é um grupo de Lie não comutativo, mas que possui uma métrica invariante à esquerda flat. Veremos aqui que a caracterização do caso flat é feita através da álgebra de Lie.

Teorema 8. Um grupo de Lie G simplesmente conexo admite métrica invariante à esquerda de curvatura nula se e somente se sua álgebra de Lie se decompõe em uma soma direta ortogonal $\mathcal{B} \oplus \mathcal{U}$ onde $\mathcal{B}$ é uma subálgebra comutativa, $\mathcal{U}$ é um ideal comutativo e a transformação linear $a d(b)$ é anti-adjunta para todo $b \in \mathcal{B}$.

Demonstração: $G$ é uma variedade Riemanniana completa de curvatura seccional constante igual a zero. Um importante resultado de geometria Riemanniana nos diz essencialmente quais são as variedades Riemannianas completas, simplesmente conexas com curvatura constante, veja por exemplo [4], pág 163. Neste caso temos que $G$ é isométrico a $\mathbb{R}^{n}$. Seja $H$ um subgrupo compacto de $G$ e consideremos a ação de $H$ em $G$ por translações à esquerda. Esta ação determina um subgrupo compacto $\tilde{H}$ do grupo de isometrias de $\mathbb{R}^{n}$ que é isomorfo a $H$. Sabemos que o grupo de isometrias do $\mathbb{R}^{n}$ é o produto semidireto de $O(n)$ por $\mathbb{R}^{n}$. Como $\tilde{H}$ é compacto, nenhum de seus elementos admite uma componente translacional. Logo, $\tilde{H} \subset O(n)$. Segue daí que $H$ possui um ponto fixo em $G$. Mas uma translação à esquerda em $G$ não trivial não possui pontos fixos, portanto $H=\{e\}$.

Consideremos a correspondência $\psi: \mathcal{G} \rightarrow \operatorname{End}(\mathcal{G})$, onde $\operatorname{End}(\mathcal{G})$ é o conjunto das transformações lineares de $\mathcal{G}$, dada por $\psi(x)=\nabla_{x}$. Seja $\langle\cdot, \cdot>$ a métrica invariante à esquerda em $G$ de curvatura $R$ identicamente nula. Lembremos que para $x, y$ e $z$ pertencentes à álgebra associada, vale que $\left\langle\nabla_{x} y, z\right\rangle=-\left\langle y, \nabla_{x} z\right\rangle$, e isto em particular implica que $\psi$ é anti-adjunta. Como a curvatura é identicamente nula, $\nabla_{[x, y]}=\nabla_{x} \nabla_{y}-\nabla_{y} \nabla_{x}$. Logo:

$$
\begin{gathered}
\psi([x, y])=\nabla_{[x, y]}=\nabla_{x} \nabla_{y}-\nabla_{y} \nabla_{x} \\
=\nabla_{x} \psi_{y}-\nabla_{y} \psi_{x}=\psi_{x} \psi_{y}-\psi_{y} \psi_{x}=\left[\psi_{x}, \psi_{y}\right] .
\end{gathered}
$$

Portanto $\psi$ é um homomorfismo de álgebras. O seu kernel, que denotaremos por $\mathcal{U}$, é um ideal, mas mais do que isto é também comutativo. De fato, a conexão $\nabla$ é simétrica, ou seja, $[x, y]=\nabla_{x} y-\nabla_{y} x$, para todo $x, y$ pertencentes à $\mathcal{G}$. Em particular, $[u, v]=\nabla_{u} v-\nabla_{v} u=0$, para $u, v \in \mathcal{U}$. Seja $\mathcal{B}$ o complemento ortogonal de $\mathcal{U}, \mathcal{B}=\mathcal{U}^{\perp}$. Para qualquer $b \in \mathcal{B}$, a transformação 
$\nabla_{b}$ leva, um elemento $\mathcal{U}$ em $\mathcal{U}$. De fato, se $u \in \mathcal{U}$, então:

$$
[b, u]=\nabla_{b} u-\nabla_{u} b=\nabla_{b} u
$$

e $[b, u] \in \mathcal{U}$ pois $\mathcal{U}$ é ideal. Portanto $\nabla_{b}$ leva $\mathcal{B}$ em $\mathcal{B}$ e assim, se $x, y \in \mathcal{B}$, então $\nabla_{x} y, \nabla_{y} x \in \mathcal{B}$ e consequentemente $[x, y] \in \mathcal{B}$, o que mostra que tal conjunto é uma subálgebra de $\mathcal{G}$. Sabemos que $\mathcal{G}$ se decompõe na soma direta ortogonal de espaços vetoriais $\mathcal{G}=\mathcal{U} \oplus \mathcal{B}$. Queremos verificar que $\mathcal{B}$ é comutativa. A aplicação $\left.\psi\right|_{\mathcal{B}}: \mathcal{B} \rightarrow \psi(\mathcal{B})$ leva $\mathcal{B}$ isomorficamente em uma subálgebra de $\mathcal{O}(n)$, formada pelas transformações de $\mathcal{G}$ anti-simétricas. Este conjunto é álgebra do grupo compacto $O(n)$, que possui uma métrica bi-invariante. Portanto qualquer subálgebra de $\mathcal{O}(n)$ pode ser equipada com uma métrica bi-invariante e, em particular, podemos equipar $\mathcal{B}$ com uma métrica bi-invariante. De acordo com a construção feita no Lema 11, podemos decompor $\mathcal{B}=\mathcal{B}_{1} \oplus \cdots \oplus \mathcal{B}_{k}$ como soma direta ortogonal de ideais, alguns simples e outros abelianos. Suponhamos que algum $\mathcal{B}_{i}$ não é comutativo. Tal $\mathcal{B}_{i}$ é uma subálgebra de $\mathcal{G}$, logo existe um único subgrupo conexo $B_{i}$ de $G$ tal que sua álgebra de Lie é $\mathcal{B}_{i}$. Este grupo é compacto, pela construção do Lema 11. Mas pelo que comentamos no início da demonstração, $B_{i}$ é trivial, logo $\mathcal{B}_{i}=\{0\}$. Segue finalmente que $\mathcal{B}$ é comutativo. Finalizando, para qualquer $b \in \mathcal{B},\left.a d(b)\right|_{\mathcal{B}}$ é anti-adjunta pois é identicamente nula e vimos que $\left.a d(b)\right|_{u}$ é dada por $\nabla_{b} u$.

Agora suponhamos que $\mathcal{G}=\mathcal{B} \oplus \mathcal{U}$ onde esta soma é ortogonal, $\mathcal{B}$ é subálgebra comutativa, $\mathcal{U}$ é um ideal comutativo e $a d(b)$ é anti-adjunta, para qualquer $b \in \mathcal{B}$. Queremos verificar que $R(x, y) z=0$, para qualquer $x, y, z \in \mathcal{G}$. Qualquer $x \in \mathcal{G}$ é escrito de maneira única como $v+b$ onde $v \in \mathcal{U}$ e $b \in \mathcal{B}$. Então, utilizando a fórmula de Koszul, temos, para $x, y \in \mathcal{G}$ quaisquer:

$$
\begin{gathered}
<\nabla_{u} x, y>=<\nabla_{u} v+\nabla_{u} b, w+\tilde{b}> \\
=<\nabla_{u} v, w>+<\nabla_{u} v, \tilde{b}>+<\nabla_{u} b, w>+<\nabla_{u} b, \tilde{b}>,
\end{gathered}
$$

observe que $\left\langle\nabla_{u} v, w\right\rangle=0$, pois $u, v, w \in \mathcal{U}$ e $\mathcal{U}$ é comutativo. Veja que:

$$
<\nabla_{u} v, \tilde{b}>=\frac{1}{2}(<[u, v], \tilde{b}>-<[v, \tilde{b}], u>+<[\tilde{b}, u], v>)
$$

e a primeira parcela se anula pois $[u, v]=0$, e as duas últimas se cancelam pois $a d(b)$ é antiadjunta para todo $b \in \mathcal{B}$. Analogamente mostramos que $\left\langle\nabla_{u} b, w\right\rangle$ e $\left\langle\nabla_{u} b, \tilde{b}\right\rangle$ são nulos. Logo $\nabla_{u} \equiv 0$. Além disso, $a d(b)(u)=[b, u]=\nabla_{b} u-\nabla_{u} b=\nabla_{b} u$ para $b \in \mathcal{B}$ e $u \in \mathcal{U}$, ou seja, $a d(b)=\nabla_{b}$.

Para concluir que o tensor de curvatura é nulo, precisamos substituir em $R(x, y) z$ elementos da forma $u+b$ e usando a trilinearidade de $R$ chegamos a algumas parcelas. Substituindo em cada parcela a fórmula da curvatura, e usando que $\nabla_{u} \equiv 0$ e $\nabla_{b}=a d(b)$ para $u \in \mathcal{U}$ e $b \in \mathcal{B}$, 
respectivamente, conclue-se que todos estes termos são nulos e portanto $R \equiv 0$. Por exemplo, $R(u, v) w=0$ para quaisquer $u, v, w \in \mathcal{U}$ pois vimos que $\nabla_{u} \equiv 0$ e o termo $R\left(\tilde{b}, b^{\prime}\right)$ também é nulo para $\tilde{b}, b^{\prime} \in \mathcal{B}$, pois

$$
R\left(\tilde{b}, b^{\prime}\right)=a d\left(\left[\tilde{b}, b^{\prime}\right]\right)-\left[a d(\tilde{b}), a d\left(b^{\prime}\right)\right]=0,
$$

e isto conclui a demonstração.

Um problema mais geral estudado por muito tempo foi encontrar exemplos de variedades Riemannianas com curvatura de Ricci identicamente nula, mas curvatura seccional não nula. Em 1953, Lichnerowicz provou que uma variedade Riemanniana conexa, compacta e homogênea com curvatura de Ricci nula é um toro euclidiano. Este resultado foi estendido para variedades homogêneas em geral por Alekseevski e Kimel'fel'd em 1975 [1] utilizando técnicas de Análise Funcional. 


\section{Grupos de Lie com curvatura estritamente positiva: Teore- mas de Wallach}

Nesta seção pretendemos estudar dois resultados interessantes envolvendo o caso de um grupo de Lie munido de métrica invariante à esquerda de curvatura estritamente positiva. Mencionamos na Seção 2 um teorema de Wallach [22] que classifica os grupos de Lie simplesmente conexos de curvatura seccional estritamente positiva. Antes de demonstrarmos tal resultado, que envolve alguns fatos sobre campos de Killing e submersões Riemannianas, estudemos o caso de curvatura escalar estritamente positiva. Para este caso, precisamos do seguinte teorema:

Teorema 9 (Iwasawa). Seja $G$ um grupo de Lie conexo. Então todo subgrupo compacto de $G$ está contido em um subgrupo compacto maximal $H$, que é necessariamente um grupo de Lie conexo. Além disso $H$ é único a menos de conjugação e $G$ é homeomorfo, como espaço topológico, ao produto $H \times \mathbb{R}^{k}$, para algum $k$.

Para a demonstração recomendamos [11]. Finalmente temos o seguinte teorema:

Teorema 10 (Wallach). Seja $G$ um grupo de Lie conexo que contém um subgrupo compacto não-comutativo. Então $G$ admite uma métrica invariante à esquerda de curvatura escalar estritamente positiva.

Demonstração: Por hipótese, $G$ contém um subgrupo compacto não comutativo. Pelo teorema de Iwasawa este subgrupo está contido em um único subgrupo compacto maximal $H$, que é um grupo de Lie conexo. Consideremos este grupo $H$. Como $H$ é compacto e conexo, existe uma métrica em $\mathcal{G}$ que é $A d-H$ invariante, isto é, invariante pela transformação adjunta $A d(h)$ : $\mathcal{G} \rightarrow \mathcal{G}$, para todo $h \in H$. Consideremos esta métrica e $\left\{e_{1}, \cdots, e_{m}\right\}$ uma base ortonormal de $\mathcal{H}$, que é uma álgebra não comutativa, pois $H$ é não comutativo e conexo. Vamos completar esta base à uma base de $\mathcal{G}$, digamos, $C=\left\{e_{1}, \cdots, e_{n}\right\}$. As transformações $\operatorname{ad}\left(e_{1}\right), \cdots, \operatorname{ad}\left(e_{m}\right)$ são anti-adjuntas, mas o resto pode não ser. Fixemos $\epsilon>0$ e vamos construir uma nova base $\tilde{C}$ fazendo:

$$
\begin{gathered}
\tilde{e}_{1}=e_{1}, \cdots, \tilde{e}_{m}=e_{m}, \\
\tilde{e}_{m+1}=\epsilon e_{m+1}, \cdots, \tilde{e}_{n}=\epsilon e_{n} .
\end{gathered}
$$

Existe uma única métrica invariante à esquerda em $G$ tal que a base $\tilde{C}$ é ortonormal. Vamos considerar agora a mesma álgebra $\mathcal{G}$ mas com esta nova métrica e, assim como fizemos na demonstração do Teorema 5 da Seção 4, olhemos para as constantes estruturais $\tilde{\alpha}_{i j k}=<\left[\tilde{e}_{i}, \tilde{e}_{j}\right], \tilde{e}_{k}>$ :

$$
\tilde{\alpha}_{i j k}=\alpha_{i j k} \quad 1 \leq i, j, k \leq m
$$




$$
\begin{aligned}
& \tilde{\alpha}_{i j k}=\epsilon \alpha_{i j k} \quad m+1 \leq i, j, k \leq n \\
& \tilde{\alpha}_{i j k}=\alpha_{i j k} \quad 1 \leq i \leq m \quad m+1 \leq j, k \leq n \\
& \tilde{\alpha}_{i j k}=\epsilon \alpha_{i j k} \quad 1 \leq j, k \leq m \quad m+1 \leq i \leq n .
\end{aligned}
$$

Temos assim definido um limite quando $\epsilon$ tende a zero e estas constantes estruturais do limite determinam uma nova álgebra de Lie que denotaremos por $\mathcal{G}_{0}$ e é dada pela soma direta ortogonal $\mathcal{H} \oplus \mathcal{U}$, onde $\mathcal{U}$ é um ideal comutativo. Existe uma métrica $\mathrm{g}_{0}$ em $\mathcal{G}_{0}$ tal que $C$ é ortonormal. Pela construção que fizemos, a aplicação adjunta $a d(h)$ é anti-adjunta para todo $h \in \mathcal{H}$. Além disso, lembrando que:

$$
\nabla_{e_{i}} e_{j}=\frac{1}{2} \sum_{k}\left(\alpha_{i j k}-\alpha_{j k i}+\alpha_{k i j}\right) e_{k}
$$

e que $\nabla$ é $\mathbb{R}$-linear, temos $\nabla_{u}=0$ para todo $u \in \mathcal{U}$ já que as constantes estruturais se anularão neste caso. Segue assim que $R_{x u}=0$, para $x \in \mathcal{G}$ e $u \in \mathcal{U}$ e consequentemente $k(x, u)=0$, para todo $x \in \mathcal{G}$ e, em particular, $r(u)=0$ em $\mathcal{U}$.

Como $\operatorname{ad}(h): \mathcal{G} \rightarrow \mathcal{G}$ é anti-adjunta para todo $h \in \mathcal{H}$, pelo Lema 3 da Seção 3 temos $r(h) \geq 0$ e a igualdade vale se e somente se $h$ é ortogonal a $[\mathcal{H}, \mathcal{H}]$, mas como $\mathcal{H}$ não é comutativo, existe $h \in \mathcal{H}$ tal que $r(h)>0$. Segue que a curvatura escalar em $\mathcal{G}_{0}$ é estritamente positiva. Como a curvatura escalar é um polinômio contínuo em função das constantes estruturais (veja. Lema 1 da Seção 2$), \rho\left(\mathcal{G}, \mathbf{g}_{\epsilon}\right)>0$ para $\epsilon$ suficientemente pequeno.

Agora pretendemos estudar o seguinte teorema:

Teorema 11 (Wallach). Seja $G$ um grupo de Lie conexo, compacto e simplesmente conexo que admite uma métrica invariante à esquerda de curvatura seccional estritamente positiva. Então $G$ é isomorfo (como grupo de Lie) a $S U(2)$.

Para estudarmos este resultado importante, precisamos de algumas definições gerais. Seja $(M,<\cdot, \cdot>)$ uma variedade Riemanniana qualquer e $X, Y$ campos de vetores $C^{\infty}$ em $M$. É possível darmos ao colchete de Lie $[X, Y]$ uma interpretação através de derivações de $Y$ ao longo de curvas integrais de $X$. Podemos dar uma interpretação parecida para campos tensoriais de $M$ (para a definição de campos tensoriais em $M$, recomendamos o Apêndice A). Se $X$ é um campo de vetores $C^{\infty}$ em $M$ e se $T$ é um $(0, s)$-tensor em $M$, então a derivada de Lie $L_{X}$ aplicada em $T$ é dada por:

$$
L_{X} T=\lim _{t \rightarrow 0} \frac{1}{t}\left[\phi_{t}^{*}(T)-(T)\right]
$$

onde $\phi_{t}$ é o fluxo local de $X$. Podemos, em particular, estudar a derivada de Lie aplicada no tensor métrico $\mathrm{g}=\langle\cdot, \cdot\rangle$. Dizemos que o campo de vetores $C^{\infty} X$ em $M$ é um campo de 
Killing se $L_{X}(\mathrm{~g}) \equiv 0$. Vamos denotar por $\nabla X$ a aplicação que a cada campo $Y$ associa o campo $\nabla_{Y} X$. Com estas notações temos a seguinte proposição:

Proposição 4. $X$ é um campo de Killing se e somente se a aplicação $\nabla X$ é anti-simétrica, isto é, $\left\langle\nabla_{Y} X, Z\right\rangle+\left\langle Y, \nabla_{Z} X\right\rangle=0$, quaisquer que sejam $Y$ e $Z$ campos de vetores $C^{\infty}$ em $M$.

Demonstração: Como $L_{X}$ é uma derivação, respeita a regra do produto. Logo:

$$
\begin{gathered}
\left(L_{X} \mathbf{g}\right)(Y, Z)=L_{X} \mathbf{g}(Y, Z)-\mathbf{g}\left(L_{X} Y, Z\right)-\mathbf{g}\left(Y, L_{X} Z\right) \\
=X<Y, Z>-<[X, Y], Z>-<Y,[X, Z]> \\
=<\nabla_{Y} X, Z>+<Y, \nabla_{Z} X>
\end{gathered}
$$

onde a última igualdade é obtida aplicando a fórmula de Koszul. Portanto $L_{X}(\mathrm{~g}) \equiv 0$ se e somente se $\left\langle\nabla_{Y} X, Z\right\rangle+\left\langle Y, \nabla_{Z} X\right\rangle=0$ para todo $Y, Z$.

Observe através da fórmula de $L_{X}$ g que se os fluxos locais $\phi_{t}$ de $X$ forem isometrias, teremos $L_{X} \mathbf{g} \equiv 0$ pois $\phi_{t}^{*} \mathbf{g}=\mathbf{g}$. Por outro lado suponhamos que $X$ é um campo de Killing, seja $\phi_{t}$ fluxo local de $X$ e $v$ um vetor tangente ao domínio do fluxo. Observe que para $s$ pequeno, o vetor $w=d \phi_{s}(v)$ também é vetor tangente ao domínio do fluxo. Portanto, pela fórmula de derivação:

$$
\lim _{t \rightarrow 0} \frac{1}{t}\left(\mathbf{g}\left(d \phi_{t} w, d \phi_{t} w\right)-\mathbf{g}(w, w)\right)=0 .
$$

Além disso, como $\phi_{s} \phi_{t}=\phi_{s+t}$, temos:

$$
\lim _{t \rightarrow 0} \frac{1}{t}\left(\mathbf{g}\left(d \phi_{s+t}(v), d \phi_{s+t}(v)\right)-\mathbf{g}\left(d \phi_{s}(v), d \phi_{s}(v)\right)\right)=0 .
$$

Isto mostra que a função real $s \mapsto \mathrm{g}\left(d \phi_{s}(v), d \phi_{s}(v)\right)$ possui derivada sempre zero e portanto é uma função constante, ou seja:

$$
\mathbf{g}\left(d \phi_{s}(v), d \phi_{s}(v)\right)=\mathbf{g}(v, v)
$$

Logo o fluxo local age por isometrias em $M$. Mostramos o seguinte:

Corolário 3. Um campo de vetores $X$ é de Killing se e somente se o grupo a um parâmetro associado a $X$ consiste em isometrias de $M$.

Seja $G$ um grupo de Lie equipado com uma métrica invariante à esquerda e $X$ um campo de vetores invariante à direita. O fluxo $\phi_{t}$ de $X$ em $G$ é, na realidade, um fluxo global tal que $\phi_{t}(g)=\phi_{t}(e) g=\exp (t X) g$, para $g \in G$ e $t \in \mathbb{R}$. Estas são isometrias globais de $G$, o que mostra que todo campo invariante à direita em $G$ é um campo de Killing. 
Em 1965, M. Berger mostrou algumas propriedades interessantes sobre campos de Killing. Estudaremos algumas delas aqui.

Lema 12 (Berger). Seja $Y$ um campo de Killing em uma variedade Riemanniana $M$ e seja $p \in M$ um ponto crítico da função

$$
q \mapsto<Y, Y>_{q}
$$

Então as seguintes igualdades se verificam para todo campo $Z$ de $M$ :

$$
\begin{gathered}
<\nabla_{Z} Y, Y>_{p}=0 \\
<\nabla_{Z} Y, \nabla_{Z} Y>_{p}=\frac{1}{2} Z_{p} Z<Y, Y>+<R(Y, Z) Y, Z>_{p} .
\end{gathered}
$$

Demonstração: Seja $f(q)=<Y, Y>_{q}$. Como $p$ é ponto crítico de $f, Z_{p}(f)=d f_{p}(Z)=0$, para todo campo Z. Portanto:

$$
0=Z_{p}(f)=2<\nabla_{Z} Y, Y>_{p}
$$

e segue a primeira igualdade do lema. Observemos agora que se $Z$ é um campo qualquer em $M$, $Z_{p}(Z f)$ depende somente do valor de $Z$ em $p$. De fato, se $W$ é outro campo tal que $W_{p}=Z_{p}$, então como $p$ é ponto crítico de $f$, observe que:

$$
W_{p}(W f)-Z_{p}(Z f)=Z_{p}(W f)-W_{p}(Z f)=[Z, W]_{p}(f)=0
$$

o que mostra que $Z_{p}(Z f)$ está unicamente determinado pelo valor de $Z$ em $p$. Além disso sabemos que $<\nabla_{Z} Y, \nabla_{Z} Y>$ e $<R(Y, Z) Y, Z>_{p}$ dependem somente do valor de $Z$ em $p$. Podemos modificar $Z$ de maneira conveniente, mantendo fixo o valor de $Z$ em $p$, de forma que podemos supor $\nabla_{v} Z=0$, para todo $v \in T_{p} M$. Utilizando todas esses fatos, temos:

$$
\begin{gathered}
\frac{1}{2} Z_{p} Z<Y, Y>=Z_{p}<\nabla_{Z} Y, Y>=Z_{p}\left(-<Z, \nabla_{Y} Y>\right) \\
=Z_{p}\left(<\nabla_{Y} Z, Y>-Y<Z, Y>\right)=Z_{p}<\nabla_{Y} Z, Y>-Z_{p} Y<Z, Y> \\
=<\nabla_{Z} \nabla_{Y} Z, Y>_{p}+<\nabla_{Y_{p}} Z, \nabla_{Z_{p}} Y>-Z_{p} Y<Z, Y> \\
=<\nabla_{Z} \nabla_{Y} Z, Y>_{p}-Z_{p} Y<Z, Y>,
\end{gathered}
$$

onde usamos que a métrica é compatível, que $\nabla Y$ é uma aplicação anti-simétrica e que $\nabla_{v} Z=0$, para todo $v \in T_{p} M$. Por outro lado:

$$
\begin{gathered}
<R(Y, Z) Y, Z>_{p}=-<R(Y, Z) Z, Y>_{p} \\
=-<\nabla_{[Y, Z]} Z-\nabla_{Y} \nabla_{Z} Z+\nabla_{Z} \nabla_{Y} Z, Y>_{p}
\end{gathered}
$$




$$
\begin{gathered}
=-<\nabla_{[Y, Z]_{p}} Z, Y_{p}>+<\nabla_{Y} \nabla_{Z} Z, Y>_{p}-<\nabla_{Z} \nabla_{Y} Z, Y>_{p} \\
=<\nabla_{Y} \nabla_{Z} Z, Y>_{p}-<\nabla_{Z} \nabla_{Y} Z, Y>_{p} .
\end{gathered}
$$

Nestas igualdades estamos novamente utilizando os mesmos fatos. Ficamos com:

$$
\frac{1}{2} Z_{p} Z<Y, Y>+<R(Y, Z) Y, Z>_{p}=-Z_{p} Y<Z, Y>+<\nabla_{Y} \nabla_{Z} Z, Y>_{p} .
$$

Desenvolvendo o último termo:

$$
\begin{gathered}
<\nabla_{Y} \nabla_{Z} Z, Y>_{p}=Y_{p}<\nabla_{Z} Z, Y>-<\nabla_{Z_{p}} Z, \nabla_{Y_{p}} Y> \\
=Y_{p}\left(Z<Z, Y>-<Z, \nabla_{Z} Y>\right) \\
=Y_{p} Z<Z, Y>-Y_{p}<Z, \nabla_{Z} Y>=Y_{p} Z<Z, Y>.
\end{gathered}
$$

A última igualdade ocorre pois como $\nabla Y$ é anti-simétrica, $\left\langle\nabla_{Z} Y, Z\right\rangle=0$. Assim temos:

$$
\frac{1}{2} Z_{p} Z<Y, Y>+<R(Y, Z) Y, Z>_{p}=Y_{p} Z<Z, Y>-Z_{p} Y<Z, Y>=[Y, Z]_{p}<Z, Y>.
$$

Por outro lado:

$$
\begin{gathered}
{[Y, Z]_{p}<Z, Y>=<\nabla_{[Y, Z]_{p}} Z, Y_{p}>+<Z, \nabla_{[Y, Z]} Y>_{p}} \\
=-<\nabla_{Z} Y,[Y, Z]>_{p}=-<\nabla_{Z_{p}} Y, \nabla_{Y_{p}} Z-\nabla_{Z_{p}} Y>=<\nabla_{Z} Y, \nabla_{Z} Y>_{p} .
\end{gathered}
$$

E finalmente encontramos a fórmula desejada.

Como consequência interessante temos o seguinte:

Corolário 4 (Berger). Seja $M$ uma variedade Riemanniana conexa, compacta de dimensão par com curvatura seccional estritamente positiva. Então todo campo de Killing $X$ em $M$ possui alguma singularidade.

Demonstração: Seja $q \in M$ e consideremos novamente a função $f: M \rightarrow \mathbb{R}$ dada por $f(q)=<X, X>_{q}$. Como a variedade $M$ é compacta, essa função possui um ponto de mínimo global $p \in M$. Suponhamos por absurdo que $X(p) \neq 0$. A aplicação $\nabla X$ associa a cada campo $Y$, o campo $\nabla_{Y} X$ e de acordo com o lema anterior, $\left\langle\nabla_{Y} X, X\right\rangle_{p}=0$, para qualquer campo $Y$ em $M$. Portanto, a aplicação $\nabla X$ induz a seguinte aplicação:

$$
\psi=\left.\nabla\right|_{V^{\perp}}: V^{\perp} \rightarrow V^{\perp}
$$

onde $V$ é a reta gerada por $X(p) \neq 0$. Agora mostremos que mais do que linear e anti-simétrica, esta aplicação é um isomorfismo. Basta verificar que $\psi$ é injetiva. Como $p$ é mínimo, $Z_{p}(Z f) \geq 0$ 
e pela hipótese de curvatura, $<R\left(X_{p}, Z_{p}\right) X_{p}, Z_{p}>$ é estritamente positivo, para $Z_{p} \in V^{\perp}, Z_{p} \neq$ 0 . Pelo lema anterior, $\left\langle\nabla_{Z} X, \nabla_{Z} X\right\rangle_{p}$ é estritamente positivo, e portanto a aplicação $\psi$ é injetiva. Mas observe que a dimensão de $V^{\perp}$ é ímpar, pois por hipótese a dimensão de $M$ é par. Mostramos então que $\psi$ é um isomorfismo anti-simétrico em um espaço vetorial de dimensão ímpar, porém isso é absurdo pois em geral se $A \in M(n, \mathbb{R})$ é inversível e $A^{t}=-A$ então:

$$
\operatorname{det} A=\operatorname{det} A^{t}=-\operatorname{det} A=(-1) \operatorname{det} A \text {. }
$$

O que implica em $n$ par.

Muitos outros resultados envolvendo curvatura e campos de Killing já foram encontrados. Em 1946 Bochner mostrou que se $M$ é compacta e possui todas as curvaturas de Ricci estritamente negativas, então todo campo de Killing em $M$ é trivial. Em 1995, X. Rong encontrou uma relação entre uma propriedade de campos de Killing com o grupo fundamental da variedade. Estes resultados fogem dos nossos propósitos e ao leitor interessado recomendamos [19] e [20].

Para a demonstração do teorema de Wallach, além dos fatos sobre campos de Killing aqui demonstrados, precisamos de alguns fatos sobre submersões Riemannianas. Consideraremos $G$ um grupo de Lie compacto, $H$ subgrupo fechado de $G$ e $H \backslash G$ o conjunto das classes laterais à direita, isto é, $H \backslash G=\{H x ; x \in G\}$. Sabemos que existe uma métrica bi-invariante em $G$ e para tal métrica existe uma única métrica Riemanniana em $H \backslash G$ tal que a projeção canônica $\pi: G \rightarrow H \backslash G$ é uma submersão Riemanniana (veja [6]). Seja $C_{G}(H)$ o grupo centralizador de $H$ em $G$, ou seja:

$$
C_{G}(H)=\{g \in G ; g h=h g, \forall h \in H\} .
$$

Para cada $g \in C_{G}(H)$, a translação à esquerda $L_{g}: G \rightarrow G$ induz uma função bem definida no quociente:

$$
\bar{L}_{g}: H \backslash G \rightarrow H \backslash G .
$$

Esta aplicação é, de fato, um difeomorfismo com inversa $\bar{L}_{g}^{-1}=\bar{L}_{g^{-1}}$ e $\bar{L}_{g} \circ \pi=\pi \circ L_{g}$. Mais do que isso, $\bar{L}_{g}$ é uma isometria de $H \backslash G$, onde a métrica definida em $H \backslash G$ que torna a projeção uma submersão Riemanniana é dada por:

$$
<<v, w>_{H g}=<\bar{v}_{g}, \bar{w}_{g}>
$$

onde $\bar{v}_{g}$ e $\bar{w}_{g}$ são os levantamentos horizontais de $v, w \in(H \backslash G)_{H g}$ em $g$, respectivamente. Provemos agora que $\bar{L}_{g}$ é uma isometria de $H \backslash G$. Veja que:

$$
\left(d \bar{L}_{x}\right)_{H y}(v)=\left(d \bar{L}_{x}\right)_{\pi(y)}(d \pi)_{y}(\bar{v})=d\left(\bar{L}_{x} \circ \pi\right)_{y}(\bar{v})=d \pi_{L_{x}(y)}\left(d L_{x}\right)_{y}(\bar{v})
$$


e isto mostra que $\left(d L_{x}\right)_{y}(\bar{v})$ é o levantamento horizontal de $\left(d \bar{L}_{x}\right)_{H y}(v)$ em $L_{x}(y)$. Portanto:

$$
<<\left(d \bar{L}_{x}\right)_{H y}(v),\left(d \bar{L}_{x}\right)_{H y}(w)>>=<\left(d L_{x}\right)_{y}(\bar{v}),\left(d L_{x}\right)_{y}(\bar{w})>=<\bar{v}, \bar{w}>_{y}=<<v, w>>.
$$

Justifica-se assim que cada $g \in C_{G}(H)$ gera uma isometria de $H \backslash G$.

Usaremos também outros resultados sobre submersões Riemannianas que enunciaremos aqui sem demonstração, mas deixamos referências precisas ao leitor interessado. Com a mesma notação anterior, primeiro temos a seguinte:

Proposição 5. Sejam $X$ e $Y$ campos de vetores em uma variedade diferenciável $M$ e sejam $\bar{X}$ $e \bar{Y}$ os levantamentos horizontais de $X$ e $Y$ em $M$, respectivamente. Então:

$$
\bar{\nabla}_{\bar{X}} \bar{Y}=\overline{\nabla_{X} Y}+\frac{1}{2}[\bar{X}, \bar{Y}]^{v}
$$

onde $[\bar{X}, \bar{Y}]^{v}$ indica a componente vertical do campo $[\bar{X}, \bar{Y}]$.

Demonstração: Recomendamos 3.55, [6].

O próximo resultado é conhecido como fórmula de O'Neill.

Proposição 6 (O'Neill). Seja $p: \bar{M} \rightarrow M$ uma submersão Riemanniana e $X, Y$ campos de vetores ortonormais em $M$ com levantamentos $\bar{X}$ e $\bar{Y}$. Sejam $K, \bar{K}$ as curvaturas seccionais em $M$ e $\bar{M}$, respectivamente. Então:

$$
K(X, Y)=\bar{K}(\bar{X}, \bar{Y})+\frac{3}{4}\left\|[\bar{X}, \bar{Y}]^{v}\right\|^{2} .
$$

Demonstração: Recomendamos 3.61, [6].

Outra definição necessária é a de toro maximal. Seja $G$ um grupo de Lie compacto e conexo. Um toro maximal T é um subgrupo de Lie fechado de $G$ que é um toro $\left(S^{1} \times \cdots \times S^{1}\right)$ e que é maximal no sentido usual, isto é, não está contido em nenhum outro toro de $G$. Observe que para $X \neq 0$ pertencente à álgebra de Lie de $G$, a aplicação:

$$
\phi: t \mapsto \exp (t X)
$$

para $t \in \mathbb{R}$ é um homomorfismo de grupos de Lie. Seu fecho em $G$, que denotaremos por $H$, é um subgrupo de Lie compacto, conexo e abeliano de dimensão $k$, digamos. Mas sabemos da teoria de grupos de Lie que nessas condições $H$ é um toro $\mathrm{T}^{k}$ (veja [16]). Em particular todo grupo de Lie conexo e compacto possui um toro unidimensional. 
Existe uma vasta linha na teoria de grupos de Lie envolvendo o estudo de toros maximais, como por exemplo a teoria de representação de grupos de Lie compactos. Para os nossos propósitos, precisaremos de um resultado que classifica grupos de Lie que possuem um toro unidimensional maximal (é equivalente dizermos que o grupo possue uma subálgebra de Cartan de dimensão 1). Usaremos aqui que $S U(2)$ é o único grupo de Lie simplesmente conexo que possui um toro maximal unidimensional. Este resultado pode ser encontrado com mais detalhes em [10], capítulo X, §6.

Finalmente podemos demonstrar o teorema de Wallach.

Demonstração do teorema 12: Lembremos que por hipótese $G$ é um grupo de Lie simplesmente conexo munido de uma métrica invariante à esquerda de curvatura seccional estritamente positiva. Como consequência do teorema de Bonnet-Myers, $G$ é compacto. Seja $T$ um toro unidimensional em $G$ (que sabemos existir, pelas observações anteriores). Consideremos agora a variedade compacta $T \backslash G$. Pela Proposição 6 (fórmula de O'Neill), $T \backslash G$ também tem curvatura seccional estritamente positiva. Vimos também que todo campo invariante à direita em $G$ é um campo de Killing e além disso nunca se anula. Pelo corolário 4, $G$ tem dimensão ímpar e todo campo de Killing na variedade $T \backslash G$ (de dimensão ímpar) se anula em algum ponto.

Vamos mostrar que $T$ é um toro maximal de $G$. Seja $C_{G}(T)$ o grupo centralizador de $T$ em $G$. Para mostrarmos que $T$ é maximal, basta verificarmos que $C_{G}(T)=T$, pois qualquer toro $T^{\prime}$ que contém $T$ está contido em $C_{G}(T)$. Para isso, basta mostrarmos que a álgebra de Lie de $C_{G}(T)$ está contida na álgebra de Lie de $T$, observando que o centralizador $C_{G}(T)$ é um grupo de Lie conexo (veja [10], pág. 287). Seja $X$ um elemento da álgebra de Lie de $C_{G}(T)$. Vamos definir um campo em $T \backslash G$ por:

$$
\tilde{X}(T g)=\left.\frac{d}{d t}\right|_{t=0} \bar{L}_{\exp (t X)}(T g) .
$$

Então o fluxo em $\tilde{X}$ é dado por $\phi_{t}(T g)=\bar{L}_{\exp (t X)}(T g)$ e vimos nos comentários antecessores desta demonstração que as transformações $\bar{L}_{\exp (t X)}$ são isometrias de $T \backslash G$. Portanto $\tilde{X}$ é um campo de Killing. Mas vimos que todo campo de Killing em $T \backslash G$ se anula em algum ponto. Então se $T g$ é um zero de $\tilde{X}$, a curva integral $\bar{L}_{\exp (t X)}(T g)$ é constante e portanto $\exp (t X) \in T$, para qualquer $t \in \mathbb{R}$ e assim $X$ é um elemento da álgebra de Lie de $T$. Logo $T$ é um toro maximal unidimensional e pelo resultado de classificação mencionado anteriormente, o único grupo de Lie compacto e simplesmente conexo que possui um toro maximal unidimensional é $S U(2)$. 


\section{Apêndice A}

Definição 1. Um tensor $T$ em um espaço vetorial $V$ sobre $\mathbb{R}$ é uma aplicação multilinear

$$
T: \underbrace{V \times \cdots \times V}_{r} \times \underbrace{V^{*} \times V^{*}}_{s} \rightarrow \mathbb{R}
$$

onde $V^{*}$ é o espaço dual a $V$. Chamamos $r$ de ordem covariante e $s$ ordem contravariante de $T$.

Costumamos dizer que $T$ é um tensor $(\dot{r}, s)$ e denotamos o conjunto de todos os tensores $(r, s)$ em $V$ por $\mathcal{T}_{s}^{r}(V)$. É possível mostrarmos que, com definições naturais de soma e multiplicação por escalar, o conjunto $\mathcal{T}_{s}^{r}(V)$ é um espaço vetorial de dimensão $n^{r+s}$. Podemos estender esta definição para variedades diferenciáveis da seguinte maneira:

Definição 2. Um campo tensorial $C^{\infty}$ de ordem $r$ em uma variedade diferenciável $C^{\infty} M$ é uma função $\Phi$ que associa a cada $p \in M$ um elemento $\Phi_{p} \in \mathcal{T}^{r}\left(T_{p} M\right)$. Além disso, dados $r$ campos de vetores $C^{\infty} X_{1}, \cdots, X_{r}$ em um aberto $U$ de $M$, então $\Phi\left(X_{1}, \cdots, X_{r}\right)=\Phi_{p}\left(X_{1}(p), \cdots, X_{r}(p)\right)$ é uma função $C^{\infty}$ em $U$. Denotamos o conjunto de todos os campos de tensores covariantes $C^{\infty}$ de ordem $r$ em $M$ por $\mathcal{T}^{r}(M)$.

Este conjunto também é um espaço vetorial sobre $\mathbb{R}$ e, além disso, um módulo sobre $C^{\infty}(M)$. O campo tensorial $\Phi \in \mathcal{T}^{r}(M)$ é dito simétrico se para cada $1 \leq i, j \leq r$ :

$$
\Phi\left(X_{1}, \cdots, X_{i}, \cdots, X_{j}, \cdots, X_{r}\right)=\Phi\left(X_{1}, \cdots, X_{j}, \cdots, X_{i}, \cdots, X_{r}\right) \text {, }
$$

e alternado se

$$
\Phi\left(X_{1}, \cdots, X_{i}, \cdots, X_{j}, \cdots, X_{r}\right)=-\Phi\left(X_{1}, \cdots, X_{j}, \cdots, X_{i}, \cdots, X_{r}\right) .
$$

Assim como é feito para o caso de um espaço vetorial, podemos definir um campo tensorial alternado em uma variedade $M$ através de permutações e sinais de permutações. Se $\sigma$ é uma permutação do conjunto $(1, \cdots, r)$, dizemos que $\Phi$ é alternado se $\Phi\left(X_{\sigma(1)}, \cdots, X_{\sigma(r)}\right)=$ $\operatorname{sgn}(\sigma) \Phi\left(X_{1}, \cdots, X_{r}\right)$. Gostaríamos também de definir o produto de campos tensoriais. Dizemos que se $\phi \in \mathcal{T}^{r}(M)$ e $\psi \in \mathcal{T}^{s}(M)$, então o produto de $\phi$ por $\psi$ é dado por:

$$
\phi \otimes \psi\left(X_{1}, \cdots, X_{r}, X_{r+1}, \cdots, X_{r+s}\right)=\phi\left(X_{1}, \cdots, X_{r}\right) \psi\left(X_{r+1}, \cdots, X_{r+s}\right) .
$$

O produto é um campo tensorial de ordem $r+s$ (é, de fato, uma função $C^{\infty}$ ). Esta aplicação é bilinear e associativa e se $\omega_{1}, \cdots, \omega_{n}$ é uma base de $\mathcal{T}^{1}(M)$, então $\left\{\omega^{i_{1}} \otimes \cdots \otimes \omega^{i_{r}} ; 1 \leq i_{1}, \cdots i_{r} \leq\right.$ 
$n\}$ é uma base de $\mathcal{T}^{r}(M)$. Lembrando o que é feito no caso de espaços vetoriais, em geral, o produto de dois tensores alternados não é um tensor alternado, diante desta situação introduzse o que é chamado de produto exterior. Um campo tensorial covariante alternado de ordem $r$ em uma variedade é chamado de forma diferencial exterior, ou somente $r$-forma. Denotando o conjunto de todas $r$-formas em $M$ por $\bigwedge^{r}(M)$, consideremos a aplicação de $\bigwedge^{r}(M) \times \bigwedge^{s}(M) \rightarrow$ $\bigwedge^{r+s}(M)$ definida por:

$$
(\phi, \psi) \mapsto \frac{(r+s) !}{r ! s !} \mathcal{A}(\phi \otimes \psi),
$$

onde $\mathcal{A}: \mathcal{T}(M)^{r} \rightarrow \mathcal{T}^{r}(M)$ é dada por:

$$
(\mathcal{A} \Phi)\left(X_{1}, \cdots, X_{r}\right)=\frac{1}{r !} \sum_{\sigma} \operatorname{sgn}(\sigma) \Phi\left(X_{\sigma(1)}, \cdots, X_{\sigma(r)}\right) .
$$

Este produto é chamado de produto exterior de $\phi$ e $\psi$ e é denotado por $\phi \wedge \psi$. Podemos mostrar que o produto exterior é bilinear, associativo e satisfaz a relação $\phi \wedge \psi=(-1)^{r s} \psi \wedge \phi$. Estes conceitos estão relacionados com a orientação de uma variedade. Em específico, definimos uma variedade $M$ como sendo orientável se é possível definir em $M$ uma $n$-forma $\omega$ que não se anula em nenhum ponto, dizemos também que $M$ é orientada pela escolha de tal $\omega$. Na realidade, $\omega$ orienta cada espaço tangente à variedade. No caso de $V$ um espaço vetorial de dimensão $n$, observe que $\operatorname{dim} \bigwedge^{n}(V)=1$, logo qualquer elemento não nulo é base deste conjunto. Ocorre que se $\left\{e_{1}, \cdots, e_{n}\right\}$ e $\left\{f_{1}, \cdots, f_{n}\right\}$ são duas bases de $V$ e se $(\alpha)_{i, j}$ é a matriz de mudança de base, então $\Omega\left(e_{1}, \cdots, e_{n}\right)=\operatorname{det}(\alpha)_{i, j} \Omega\left(f_{1}, \cdots, f_{n}\right)$, onde $\Omega$ é um tensor covariante alternado de ordem $n=\operatorname{dim} V$. Poranto, $\Omega$ tem mesmo sinal em duas bases se o determinante de mudança é positivo e tem sinal contrário no outro caso, de maneira que a escolha de tal $\Omega$ determina uma orientação para $V$. Dois tensores $\Omega_{1}, \Omega_{2}$ determinam a mesma orientação se e somente se $\Omega_{1}=\lambda \Omega_{2}$, onde $\lambda$ é um real positivo. O mesmo ocorre em uma variedade diferenciável $M$, com algumas diferenças. Existe outra definição de orientabilidade em variedades, mais intuitiva. Ela diz que uma variedade $M$ é orientável se pode ser coberta por uma coleção de vizinhanças coordenadas tais que se $U_{\alpha} \cap U_{\beta} \neq \emptyset$, então $\phi_{\alpha} \circ \phi_{\beta}^{-1}$ possui Jacobiano positivo. É possível mostrar que as duas definições coincidem. Estas definições são as mesmas se estamos lidando com uma variedade Riemanniana. A vantagem no caso de orientabilidade é que em uma variedade Riemanniana $M$ orientável existe uma única $n$-forma $\omega$ que vale +1 em toda base ortonormal orientada. Esta tal forma é chamada de elemento de volume de $M$.

Com o conceito de $n$-forma em uma variedade diferenciável $M$, podemos definir a integral de uma $n$-forma $\omega$ em $M$, denotada por $\int_{M} \omega$. Mais ainda, com o conceito de métrica, em uma variedade Riemanniana podemos definir a integral de uma função sobre um domínio de integração. 
Agora seja $M$ uma variedade diferenciável orientada, e $n=\operatorname{dim} M$. Portanto existe uma $n$ forma (um elemento de volume) $\Omega$ que nunca se anula em $M$. Além disso, qualquer outra $n$-forma $\omega$ é dada por $\omega=f \Omega$, onde $f$ é uma função em $M$ ( $\omega$ tem a mesma classe de diferenciabilidade de $f$ ). Uma função $f$ em $M$ é dita integrável se é limitada, tem suporte compacto e é contínua exceto em um conjunto de medida nula (dizemos quase-contínua). Uma $n$-forma $\omega$ em $M$, como função que associa a cada $p \in M$ um elemento $\omega_{p} \in \Lambda^{n}\left(T_{p}(M)\right)$ é dita integrável se $\omega=f \Omega$, onde $f$ é uma função integrável. Então seja $\omega$ uma $n$-forma em $M$ integrável e tal que seu suporte está contido no interior de um cubo $Q \subset M$, isto é, $Q$ está contido no domínio de uma vizinhança coordenada orientada $\left(U, \phi \sim\left(x^{1}, \cdots, x^{n}\right)\right)$ tal que $\phi(Q)=C$, onde $C$ é o cubo unitário em $\mathbb{R}^{n}$ dado por $\left\{x \in \mathbb{R}^{n} ; 0 \leq x^{i} \leq 1, i=1, \cdots, n\right\}$. Seja $(U, \phi)$ vizinhança coordenada associada a $Q$ e suponhamos que a expressão $\phi^{-1 *}(\omega)=f(x) d x^{1} \wedge \cdots \wedge d x^{n}$ é a representação de $\omega$ em coordenadas locais. Então $f$ é limitada e quase-contínua em $C$, de maneira que $\int_{C} f d v$, onde $d v=d x^{1} \wedge \cdots \wedge d x^{n}$, está definida. Temos assim:

$$
\int_{M} \omega=\int_{C} f d v
$$

Para definirmos $\int_{M} \omega$ quando o suporte de $\omega$ não está contido em uma carta, usamos uma partição da unidade $\left\{f_{\alpha}\right\}$ subordinada à cobertura $\left\{U_{\alpha}\right\}$ e decompomos $\omega$ em uma soma finita de $n$-formas com suporte contido em cada $\left\{U_{\alpha}\right\}$. É possível mostrar que esta integral acima é independente de uma escolha particular de cubo $Q$ e que satisfaz algumas propriedades. Por exemplo, ela muda de sinal se mudamos a orientação da variedade e a aplicação $\omega \mapsto \int_{M} \omega$ é $\mathbb{R}$-linear. Além disso, se $F: M_{1} \rightarrow M_{2}$ é um difeomorfismo e $\omega$ é uma 2-forma em $M_{2}$, então:

$$
\int_{M_{1}} F^{*} \omega= \pm \int_{M_{2}} \omega
$$

onde o sinal depende se $F$ é um difeomorfismo que preserva ou reverte orientação.

Agora seja $M$ uma variedade Riemanniana orientável e seja $\Omega$ a orientação escolhida. Pelo que vimos acima, qualquer outra $n$-forma em $M$ é dada por $f \Omega$, onde $f$ é uma função $C^{\infty}$ em $M$. Definindo mais precisamente, se $D$ é um domínio de integração em $M$, e $k_{D}$ é a função característica de $D$, definimos o volume de $D$ por $\operatorname{vol}(D)=\int_{M} k_{d} \Omega$. Se $f$ é uma função em $M$ limitada e contínua exceto em um conjunto de medida nula, a integral de $f$ sobre $D$ é definida por $\int_{D} f=\int_{M} f k_{D} \Omega$. Se $M$ é compacta, temos $\operatorname{vol}(M)=\int_{M} \Omega$ e $\int_{M} f=\int_{M} f \Omega$. Esta definição de fato faz sentido pois $k_{D}$ é contínua exceto da fronteira de $D$, que possui conteúdo nulo, e possui suporte compacto. É possível mostrar que esta integral definida satisfaz as mesmas propriedades da integral de Riemann em $\mathbb{R}^{n}$.

Tudo fica mais interessante quando a variedade em questão é um grupo de Lie. Então seja $G$ um grupo de Lie de dimensão $n$. Um campo tensorial covariante de ordem $r \Phi$ em $G$ é chamado 
de invariante à esquerda (direita) se $L_{a}^{*} \Phi_{a g}=\Phi_{g}\left(R_{a}^{*} \Phi_{g a}=\Phi_{g}\right)$. Dizemos que $\Phi$ é bi-invariante se é invariante à esquerda e à direita. Observemos que todo campo tensorial covariante invariante à esquerda (ou direita) em $G$ é, de fato, $C^{\infty}$. Se $\left\{X_{1}, \cdots, X_{n}\right\}$ é uma base de campos de vetores $C^{\infty}$ invariantes à esquerda, então:

$$
\left(L_{a}^{*} \Phi\right)_{g}\left(\left(X_{1}\right)_{g}, \cdots,\left(X_{n}\right)_{g}\right)=\Phi_{a g}\left(d L_{a}\left(X_{1}\right), \cdots, d L_{a}\left(X_{n}\right)\right)=\Phi_{g}\left(X_{1}, \cdots, X_{n}\right)
$$

O primeiro termo é igual a $\Phi_{g}\left(\left(X_{1}\right)_{g}, \cdots,\left(X_{n}\right)_{g}\right)$. Se tomarmos então $g=e$, temos que o termo $\Phi\left(X_{1}, \cdots, X_{n}\right)$ é constante e, portanto, $C^{\infty}$. Temos que as componentes de $\Phi$ com relação a. qualquer base $C^{\infty}$ são constantes e, portanto, $C^{\infty}$. Mostraremos agora algumas propriedades parecidas com o que fizemos com métricas invariantes à esquerda, o que é esperado pois a métrica é um campo tensorial covariante de ordem 2 .

Seja $\Phi_{e}$ um tensor covariante de ordem $r$ em $T_{e} G$. Para cada $g \in G$, existe uma única translação à esquerda $L_{g}$ que leva $e$ em $g$. Podemos assim definir $\Phi_{g}=L_{g^{-1}}^{*} \Phi_{e}$ um campo tensorial de ordem $r$ em $G$ que coincide com $\Phi_{e}$ em $e$. Não é difícil verificar que este campo assim definido é invariante à esquerda. Analogamente podemos definir $R_{g^{-1}}^{*} \Phi_{e}$ e mostrar que tal campo é invariante à direita. Assim como fizemos na Proposição 1 da Seção 2, podemos mostrar aqui que $\Phi_{e}$ determina um tensor bi-invariante se e somente se $A d(g)^{*} \Phi_{e}=\Phi_{e}$, para todo $g \in G$. Se $\Phi$ é bi-invariante, então $\operatorname{Ad}(g)^{*} \Phi_{e}=L_{g}^{*} \circ R_{g^{-1}}^{*} \Phi_{e}=\Phi_{e}$. Ao mesmo tempo, se tal igualdade vale, então:

$$
L_{g^{-1}}^{*} \Phi_{e}=L_{g^{-1}}^{*} \circ L_{g}^{*} \circ R_{g^{-1}}^{*} \Phi_{e}=R_{g^{-1}}^{*} \Phi_{e} .
$$

$\mathrm{O}$ primeiro e último termo dessa igualdade são os campos invariante à esquerda e à direita determinados por $\Phi_{e}$, respectivamente. Mostramos que eles coincidem em todo $g \in G$, o que conclui o que afirmamos.

Como consequência, todo grupo de Lie é orientável. A escolha de um produto interno e de uma orientação em $T_{e} G$ determinam um elemento de volume $\Omega_{e}$ neste espaço que pode ser estendido (pelo que mencionamos acima) a um elemento de volume $\Omega$ em $G$. Finalmente chegamos ao principal resultado deste apêndice.

Teorema 12. Seja $G$ um grupo de Lie compacto e conexo. Então $G$ possui um único elemento de volume bi-invariante $\Omega$ tal de $\operatorname{vol}(G)=1$.

Demonstração: Seja $\Omega$ um elemento de volume invariante à esquerda em $G$. Queremos mostrar que $\Omega$ é também invariante à direita. Pelo que mencionamos anteriormente, basta mostrarmos que $A d(g)^{*} \Omega_{e}=\Omega_{e}$, para todo $g \in G$. Seja $X_{1}, \cdots, X_{n}$ uma base de $\mathcal{G}$. Consideremos a representação adjunta de $G, A d: g \in G \rightarrow A d(g) \in A u t(\mathcal{G})$, onde $A u t(\mathcal{G})$ é o conjunto de 
automorfismos de $\mathcal{G}$. Seja $\left(\alpha_{i j}(g)\right)$ a matriz de $\operatorname{Ad}(g)$ com relação à base $X_{1}, \cdots, X_{n}$. Podemos escrever $A d(g) X_{j}=\sum_{i} \alpha_{i j}(g) X_{i}$ e mostrar que a aplicação de $G$ em $G l(n, \mathbb{R})$ definida por $g \mapsto\left(\alpha_{i j}(g)\right)$ é um homomorfismo $C^{\infty}$. Além disso:

$$
\operatorname{Ad}(g)^{*} \Omega_{e}=\operatorname{det}\left(\alpha_{i j}(g)\right) \Omega_{e}
$$

A aplicação de $G$ em $\mathbb{R}^{*}$ (grupo multiplicativo dos reais exceto o zero) dada por $g \mapsto \operatorname{det}\left(\alpha_{i j}(g)\right)$ é um homomorfismo $C^{\infty}$ e portanto sua imagem é um subgrupo compacto e conexo de $\mathbb{R}^{*}$. Mas isso implica que tal imagem é $\{+1\}$, logo $\operatorname{det}\left(\alpha_{i j}(g)\right)=1$ e $A d(g)^{*} \Omega_{e}=\Omega_{e}$, para todo $g \in G$ e isto mostra que $\Omega$ é bi-invariante.

Qualquer outra forma bi-invariante é escrita como $\lambda \Omega$ onde $\lambda$ é uma constante real estritamente positiva. Assim

$$
\operatorname{vol}(G)=\int_{G} \lambda \Omega=\lambda \int_{G} \Omega
$$

de maneira que podemos escolher uma única constante $\lambda \neq 0$ tal que $\operatorname{vol}(G)=1$. 


\section{Referências}

[1] D. V. Alekseevski e B. N. Kimel'fel'd, Structure of homogeneous Riemann spaces with zero Ricci curvature, Funct. Analysis and Appl. 9 (1975), 97-102.

[2] R. Azencott e E. Wilson, Homogeneous manifolds with negative curvature, Part I., Trans. Amer. Math. Soc. 215 (1976), 323-362.

[3] W. M. Boothby, An Introduction to Differentiable Manifolds and Riemannian Geometry, Academy Press, New York, 1986.

[4] M. P. do Carmo, Geometria Riemanniana, Projeto Euclides, $2^{\circ}$ edição, Rio de Janeiro, 1988.

[5] É. Cartan, Sur la structure des groupes de transformations finis et continus, Oeuvres Complètes I, 137-192.

[6] S. Gallot, D. Hulin, J. Lafontaine, Riemannian Geometry, Springer-Verlag, França, 1993.

[7] R. Hamilton, Three-manifolds with positive Ricci curvature, J. Diff. Geometry 17 (1982) 255-306.

[8] T. Hawkins, Emergence of the Theory of Lie Groups, An Essay in the History of Mathematics 1869-1926, Springer-Verlag, New York, 2000.

[9] E. Heintze, On homogeneous manifolds of negative curvature, Math. Ann. 211 (1974) 23-34.

[10] S. Helgason, Differential Geometry and Symmetric Spaces, Academic Press, New York, 1962.

[11] K. Iwasawa, On some types os topological groups, Ann. Math. 50 (1949), 507-558.

[12] W. Killing, Die Zusammensetzung der stetigen endlichen Transformationsgruppen, I, II, III, IV, Math. Annalen, 31 (1886), 252-290; 33 (1888), 1-48; 34 (1889), 57-1222; 36 (1890), 161-189.

[13] F. Klein e S. Lie, Sur une certaine famille de courbes et de surfaces, C.R. Acad. Sci. Paris, 70 (1870), 1222-1226.

[14] F. Klein e S. Lie, Sur une certaine famille de courbes et de surfaces, C.R. Acad. Sci. Paris, 70 (1870), 1275-1279. 
[15] S. Lie (com colaboração de F. Engel), Theorie de Transformationsgruppen, 3 volumes, Teubner, Leipzig , 1888, 1890, 1893.

[16] Y. Matsushima, Differential Manifolds, Marcel Dekker, Inc., New York, 1972.

[17] J. Milnor, Curvatures of left invariant metrics on Lie groups, Adv. Math., 21 (1976), 293329.

[18] B. O'Neill, Semi-Riemannian Geometry, Academi Press, New York, 1983.

[19] P. Petersen, Riemannian Geometry, Springer-Verlag, New York, 1997.

[20] X. Rong, The almost cyclicity of the fundamental groups of positively curved manifolds, Invt. Math, 126 (1996), 47-64.

[21] V. S. Varadarajan, Lie Groups, Lie Algebras and their Representations, Prentice-Hall, New Jersey, 1974.

[22] N. Wallach, Compact homogeneous Riemannian manifolds with stricly positive curvature, Ann. Math.. 96 (1972), 277-295.

[23] F. Warner, Foundations of Differentiable Manifolds and Lie Groups, Springer-Verlag, New York, 1983.

[24] H. Weyl, Zur Theorie der Darstellung der einfachen kontinuierlichen Gruppen, Sitzungsber. Preuss. Akad. Wiss. Berlin Phys.-Math. Kl. (1924), 338-345.

[25] H. Weyl, Das gruppentheoretische Fundament der Tensorrechnung, Nachr. Ges. Wiss. Gött Phys.-Math. Kl. (1924), 218-224. 